NBER WORKING PAPER SERIES

LONG-TERM CARE HOSPITALS: A CASE STUDY IN WASTE

\author{
Liran Einav \\ Amy Finkelstein \\ Neale Mahoney \\ Working Paper 24946 \\ http://www.nber.org/papers/w24946 \\ NATIONAL BUREAU OF ECONOMIC RESEARCH \\ 1050 Massachusetts Avenue \\ Cambridge, MA 02138 \\ August 2018, Revised April 2019
}

We thank Jeremy Kahn, Mark Miller, Hannah Wunsch, and many seminar participants for helpful comments. We are grateful to Abby Ostriker and Anna Russo for excellent research assistance. Einav and Finkelstein gratefully acknowledge support from the NIA (R01 AG032449). Mahoney acknowledges support from the Becker Friedman Institute at the University of Chicago and the National Science Foundation (SES-1730466). The views expressed herein are those of the authors and do not necessarily reflect the views of the National Bureau of Economic Research.

At least one co-author has disclosed a financial relationship of potential relevance for this research. Further information is available online at http://www.nber.org/papers/w24946.ack

NBER working papers are circulated for discussion and comment purposes. They have not been peer-reviewed or been subject to the review by the NBER Board of Directors that accompanies official NBER publications.

(C) 2018 by Liran Einav, Amy Finkelstein, and Neale Mahoney. All rights reserved. Short sections of text, not to exceed two paragraphs, may be quoted without explicit permission provided that full credit, including $($ ) notice, is given to the source. 
Long-Term Care Hospitals: A Case Study in Waste

Liran Einav, Amy Finkelstein, and Neale Mahoney

NBER Working Paper No. 24946

August 2018, Revised April 2019

JEL No. H51,I11,I18

\begin{abstract}
$\underline{\text { ABSTRACT }}$
There is substantial waste in U.S. healthcare, but little consensus on how to identify or combat it. We identify one specific source of waste: long-term care hospitals (LTCHs). These post-acute care facilities began as a regulatory carve-out for a few dozen specialty hospitals, but have expanded into an industry with over 400 hospitals and \$5.4 billion in annual Medicare spending in 2014. We use the entry of LTCHs into local hospital markets and an event study design to estimate LTCHs' impact. We find that most LTCH patients would have counterfactually received care at Skilled Nursing Facilities -post-acute care facilities that provide medically similar care to LTCHs but are paid significantly less - and that substitution to LTCHs leaves patients unaffected or worse off on all dimensions we can objectively measure. Our results imply that Medicare could save about $\$ 4.6$ billion per year - with no harm to patients - by not allowing for discharge to LTCHs.

Liran Einav

Stanford University

Department of Economics

579 Serra Mall

Stanford, CA 94305-6072

and NBER

leinav@stanford.edu

Amy Finkelstein

Department of Economics, E52-442

MIT

50 Memorial Drive

Cambridge, MA 02142

and NBER

afink@mit.edu

Neale Mahoney

Booth School of Business

University of Chicago

5807 South Woodlawn Avenue

Chicago, IL 60637

and NBER

Neale.Mahoney@chicagobooth.edu
\end{abstract}




\section{Introduction}

Healthcare spending is one of the largest fiscal challenges facing the U.S. federal government. In 2014, the U.S. federal government spent $\$ 1.1$ trillion on public healthcare programs (BEA, 2016) and the CBO projects that spending will grow to $\$ 2$ trillion by 2026 (CBO, 2016).

An idée fixe in health policy is that there is significant "waste" in the U.S. healthcare system, with the widely repeated claim that 30\% of U.S. healthcare spending is wasteful (e.g., Orszag, 2009; McGinnis et al., 2013). ${ }^{1}$ Prominent stylized facts in support of this view are that the U.S. spends a much higher fraction of GDP on healthcare relative to other OECD countries but obtains only middling health outcomes (e.g., OECD, 2017; Anderson et al., 2005; Papanicolas, Woskie and Jha, 2018), and the Dartmouth Atlas findings of large unexplained variation in Medicare spending within the U.S. that is not correlated with better health outcomes (e.g., CBO, 2008; Skinner, 2011). While there is no universal definition, commentators typically use the term waste to refer to healthcare spending that does not improve patient health. Waste thus includes both transfers (e.g., excess payments to drug manufacturers) and deadweight loss (e.g., from use of an expensive technology that does not improve health).

The near-consensus on the existence of waste is, unfortunately, not matched by any agreement on how to reduce that waste. For example, Doyle, Graves and Gruber (2015) write: “There is widespread agreement that the United States wastes up to one-third of health care spending, yet pinpointing the source of the waste has proven difficult." In a similar spirit, Cutler (2010) notes: "Analysts from the left and right sides of the political spectrum agree that health care costs could be greatly reduced. There is, however, less agreement about the best strategy for reducing them."

Cutting healthcare spending is easy - closing down hospitals would do the trick. Cutting healthcare spending without harming patient health or well-being, however, has proved a much more elusive goal. There are, of course, oft-cited specific examples, such as robotic surgery tools or protonbeam therapy for prostate cancer (Chandra and Skinner, 2012). But we know of few empirical studies that have compellingly identified a specific and substantial source of waste.

In this paper we provide a case study where, our evidence suggests, a substantial amount of healthcare spending can be saved without harming patient outcomes. Our case study is of a specific

\footnotetext{
${ }^{1}$ For instance, McGinnis et al. (2013) was picked up by many major media outlets. See https://khn . org/morningbreakout/iom-report/for a summary.
} 
healthcare institution: long-term care hospitals (LTCHs). ${ }^{2}$ LTCHs are one of several types of healthcare institutions that provide post-acute care (PAC) - formal care provided to help patients recover from a surgery or other acute care event.

PAC is an under-studied sector, with large stakes for both federal spending and for patient health. Federal spending on PAC through the Medicare program is substantial, about $\$ 59$ billion in 2014. A recent Institute of Medicine report found that, despite accounting for only 16\% of Medicare spending, PAC contributed to a striking $73 \%$ of the unexplained geographic variation in Medicare spending (IOM, 2013), suggesting that there may be inefficiency in the sector.

Traditionally, PAC was provided at skilled nursing facilities (SNFs) or at home by home health agencies (HHAs). LTCHs were administratively created in the early 1980s to protect 40 chronic disease hospitals from the new Prospective Payment System introduced for acute care hospitals. What began as a regulatory carve-out for a few dozen specialty hospitals subsequently expanded into an industry with over 400 LTCHs and \$5.4 billion in annual Medicare spending in 2014 (MedPAC, 2016).

The institutional history of LTCHs - which we discuss in detail below - suggests that they may be primarily cost-increasing institutions. LTCHs are administrative - not medical - constructs. They are unique to the U.S. health care system, and, to the best of our knowledge, do not exist in any other country. LTCHs are reimbursed at substantially higher rates than other PAC facilities and are run primarily by large for-profit chains. They have also been the subject of several decades worth of a regulatory game of whack-a-mole; in a series of reforms, the Centers for Medicare and Medicaid Services (CMS) has made multiple attempts to eliminate the loopholes that LTCHs offer for excess reimbursement, and to limit the growth of the sector as a whole.

We analyze the impact of a patient being discharged to an LTCH (hereafter, "LTCH discharge") on various outcomes. Our empirical strategy is to instrument for LTCH discharge with an event study design based on the first entry of an LTCH into a local hospital market. We define hospitals markets based on Hospital Service Areas (HSAs), of which there are about 3,400 in the U.S. We analyze 16 years of data, from 1998-2014. During this period, 186 hospital markets experienced their first LTCH entry. Another 152 markets already had an LTCH at the start of our sample period, and over 3,000 markets still had no LTCH at the end of our sample period. Markets with LTCHs are disproportionately large, accounting for $34 \%$ of the Medicare enrollees by the end of our sample period.

We estimate that about four-fifths of discharges to LTCHs represent substitution from SNFs, while

\footnotetext{
${ }^{2}$ The acronym LTCH is typically pronounced"el-tack", presumably reflecting the fact that LTCHs are sometimes referred to as long-term acute care hospitals (LTACs), which is phonetically pronounced in this manner.
} 
the others substitute mostly from discharges home without home health care. SNFs are reimbursed by Medicare at substantially lower rates than LTCHs; on a per day basis, LTCHs in 2014 were reimbursed about $\$ 1,400$, compared to about $\$ 450$ for SNFs (authors' calculation based on Medicare data described below). We estimate that a discharge to an LTCH increases net Medicare spending by about $\$ 30,000$. Patients, however, do not benefit from this increased spending. Patients discharged to an LTCH owe more money out of pocket, and we find no evidence that they spend less time in institutional care or have better mortality outcomes as a result. Taken together, our findings indicate that Medicare could save roughly $\$ 4.6$ billion per year with no harm to patients by not allowing for discharge to LTCHs.

Our strategy allows us to look not only at aggregate effects of LTCHs but also at whether there is a subset of patients or LTCHs for whom the benefits of LTCH discharge are higher and/or the costs of LTCH discharge are lower. We explore potential heterogeneity across a number of natural patient and LTCH characteristics, and find little evidence of heterogeneous effects. Perhaps most interestingly, we examine heterogeneity across LTCH patients affected by a recent policy change that occurred shortly after the last year of our sample. To try to reduce expenditures and limit LTCH care to only the most clinically complex patients, in 2016 CMS announced a "dual payment structure" under which LTCHs are only reimbursed as LTCHs if the patients meet certain clinical criteria (MedPAC, 2017a). We find no evidence of lower spending impacts or of mortality benefits for these more complex patients who would continue to qualify for LTCH reimbursement under these new rules.

In interpreting our results, it is important to note that despite high short-term mortality rates in the affected population, the confidence intervals on our mortality results do not allow us to conclusively reject economically meaningful improvements in health; this is a common feature of nearly all research that considers mortality as an outcome. In addition, we are not able to measure non-mortality dimensions of health (such as pain, functional limitations, and other quality-of-life metrics) or nonhealth dimensions of utility (such as the quality of the room and board provided). Again, this is a common feature of nearly all health economics research on patient outcomes. Our analysis makes clear that any such unmeasured health or utility benefits would need to be valued at over $\$ 30,000$ per LTCH stay to cover the incremental Medicare spending associated with LTCH discharge. Moreover, the institutional history of the LTCH sector as a regulatory carve-out - rather than an institution created to serve a medical need - suggests that the "burden of proof" should be to show that LTCHs provide medical or non-medical benefits that justify their costs. Consistent with CMS' various attempts to limit the growth of LTCHs, we cannot reject the null that the medical care LTCHs provide is 
not better than the alternative.

Our paper relates to several distinct literatures. Most narrowly, it complements recent work suggesting that the PAC sector is a fruitful part of the healthcare system in which to look for inefficiencies in Medicare spending (IOM, 2013; Doyle, Graves and Gruber, 2017); relatedly, Curto et al. (2019) note that hospital patients enrolled in Medicare Advantage are much less likely to be discharged to PAC, and particularly institutional PAC (LTCHs, SNFs, and IRFs). Our results are consistent with this existing impression and point to a particular PAC institution - the LTCH - whose elimination could save money without any apparent harm to patients.

Our paper also contributes to a small but growing literature on the impact of providers on the healthcare sector. Much of this literature has focused on the effect of financial incentives on provider behavior (e.g., Cutler, 1995; Clemens and Gottlieb, 2014; Ho and Pakes, 2014; Eliason et al., 2018; Einav, Finkelstein and Mahoney, 2018), or more broadly on the role of the physician in affecting healthcare decisions (e.g., Barnett, Olenski and Jena, 2017; Molitor, 2018). Our study is unusual in that it studies the impact of a specific institution (or organizational form) on the efficiency of the healthcare sector. Most closely related to our analysis is Kahn et al. (2013) who look cross-sectionally at how outcomes for chronically ill, acute care hospital patients differ in markets with differential LTCH penetration. Like us, they conclude that increased probability of LTCH transfer is associated with lower use of SNFs, higher overall Medicare payments, and no improvement in survival; our empirical analysis below suggests, however, that there are likely confounds to such cross-sectional analysis.

The rest of our paper is organized as follows. Section 2 provides background on Post-Acute Care and LTCHs. Section 3 describes our data and presents summary statistics. Section 4 describes our empirical strategy and Section 5 reports the results. Section 6 concludes.

\section{Setting}

\subsection{Post-Acute Care}

LTCHs are part of the post-acute care (PAC) sector, which provides patients with rehabilitation and palliative services following an acute care hospital (hereafter, ACH or "hospital") stay. PAC includes both facility-based care - skilled nursing homes (SNF), inpatient rehabilitation facilities (IRFs), and long-term care hospitals (LTCHs) - and home-based care, provided by home health agencies (HHAs). About two-fifths of Medicare hospital patients are discharged to PAC, of which about $60 \%$ are sent to PAC facilities (70\% of PAC spending) and about $40 \%$ are sent to home health care (30\% of PAC 
spending) (MedPAC, 2015b). Because IRFs are institutionally similar to SNFs, but are much smaller in number, we lump them together with SNFs in our discussion and empirical analysis that follow. ${ }^{3}$

Spending on PAC is substantial. In 2014, Medicare spent $\$ 59$ billion on PAC. This is approximately $16 \%$ of the $\$ 376$ billion in total Traditional Medicare (hereafter, "Medicare") spending and about 20\% more than the much-studied Medicare Part D program spending on Traditional Medicare beneficiaries. ${ }^{4}$ PAC patients are high-risk, with $15 \%$ of Medicare deaths involving a PAC stay in the prior 30 days (Einav, Finkelstein and Mahoney, 2018). Medicare spending on PAC is growing at two percentage points faster per year than overall Medicare spending, and more than doubled between 2001 and 2013 (Boards of Trustees for Medicare, 2002, 2014). This spending growth has not been associated with any measurable improvements in patient health or quality of care (MedPAC, 2015a).

Within the PAC landscape, LTCHs generally provide the most intensive care, SNFs and IRFs provide intermediate levels of care, and HHAs provide the least intensive care. Patient health follows a similar ordering, with 90-day post-discharge mortality declining from $28 \%$ for patients discharged to LTCHs to 13\% for patients discharged to home health care in 2014.

Medicare reimbursement differs greatly across PAC providers. Loosely speaking, LTCHs are paid a fixed amount per admission, SNFs are reimbursed on a per diem basis, and HHAs are reimbursed per 60-day episode of care. In 2014, the average LTCH stay was 26 days and cost Medicare \$36,000; by contrast, an average SNF stay was 25 days and cost $\$ 12,000$. On a per day basis, therefore, LTCHs are the most expensive form of PAC (\$1,436 per day), followed by SNFs (\$466 per day), and then HHAs (\$73 per day). Patient cost sharing also differs across PAC providers. Cost sharing for LTCH stays is tied to the beneficiary's inpatient cost sharing; SNF stays are covered by a separate cost-sharing schedule, with daily copays that kick in after 20 days; and cost sharing is generally not required for HHA services.

Despite these very different reimbursement regimes, physicians lack precise medical guidelines or strict requirements from Medicare on which provider is most appropriate for a given patient, with discharge decisions reflecting non-clinical factors, such as geographic availability, patient or physician preferences, and familiarity between the PAC provider and the referring hospital (Buntin, 2007; Ottenbacher and Graham, 2007). This results in substantial overlap in the types of cases treated by different

\footnotetext{
${ }^{3}$ In 2014, there were approximately 205,000 IRF stays (\$3.3 billion in Medicare payments) and 2.5 million SNF stays (\$32.4 billion in Medicare payments). These and subsequent numbers in this section without an explicit citation are based on the Medicare data described in the next section.

${ }^{4}$ In particular, we estimate Part D spending for Traditional Medicare beneficiaries as the product of $\$ 78.1$ billion in total Part D spending (Boards of Trustees for Medicare, 2015) and the 62\% of Part D beneficiaries enrolled in stand-alone PDP plans (MedPAC, 2015b), which yields $\$ 48.4$ billion in Part D spending.
} 
PAC providers, and in significant variation in PAC utilization.

\subsection{Whack-a-Mole: A Brief Regulatory History of LTCHs}

Our analysis focuses on the impact of discharge to an LTCH. Unlike other medical facilities, LTCHs are a purely regulatory phenomenon and are unique to the U.S. health care system. In order to be classified as an LTCH, a hospital must have an average length of stay of 25 days or more. Because there are no specific medical requirements, LTCHs provide a diverse range of services, including those to address respiratory issues, septicemia, skin ulcers, and renal failure (MedPAC, 2018).

LTCHs account for about $4 \%$ of discharges to facility-based PAC and about $12 \%$ of facility-based PAC spending (MedPAC, 2015b). As we discuss in more detail below, LTCHs exist in some hospital markets but not in others; in 2014, in markets where they exist, LTCHs accounted for $11 \%$ of discharges to facility-based PAC and 31\% of facility-based PAC spending. About half of LTCHs are known as "hospitals within hospitals" meaning that they are physically located within the building or campus of a (typically larger) acute care hospital (Office of Inspector General, 2013).

The history of LTCHs reads like a whack-a-mole history of health care reform. In 1982, the Tax Equity and Fiscal Responsibility Act (TEFRA) established a prospective payment system (PPS) for acute care hospitals. Rather than being reimbursed on a fee-for-service ("cost-plus") basis, hospitals would be paid a predetermined, fixed amount that depended on the patient's diagnosis related group (DRG). At the time, there were about 40 hospitals - primarily former tuberculosis and chronic disease facilities - that specialized in clinically complex patients who required long hospital stays; regulators were concerned that the fixed payments under PPS would be insufficient to cover costs at these hospitals. To keep these hospitals afloat, CMS excluded hospitals with average length of stay of at least 25 days from PPS and continued to pay them based on their average per-diem cost (Liu et al., 2001). These 40 hospitals were the original LTCHs.

Figure 1 plots the number of LTCHs over time. Since 1982, there has been a rapid growth in the LTCH sector, with the number of facilities rising from 40 to over 400 . Because new entrants did not have prior cost data, payments for new entrants were determined by costs in their initial years of operation. This encouraged new entrants to be inefficient when they first opened and to earn profits by increasing their efficiency over time. ${ }^{5}$

The majority (72\% in 2014) of LTCHs are for-profit (21\% are non-profit and $7 \%$ are government-

\footnotetext{
${ }^{5}$ Liu et al. (2001) describes the early history and institutional features of LTCHs in greater detail.
} 
run). ${ }^{6}$ According to recent financial statements of the two largest LTCH operators, Kindred Health Systems and Select Medical, LTCHs generate profits margins between 16\% and 29\%. ${ }^{7}$

Since their creation in 1982, a series of policies have been enacted to try to curb rising LTCH expenditures. The 1997 Balanced Budget Act (BBA) and 1999 Balanced Budget Refinement Act (BBRA) established a prospective payment system for LTCHs. From 2002 to 2007, LTCHs were transitioned to a payment system in which, like the PPS for acute care hospitals, they were paid a fixed amount per patient-DRG. However, much like LTCHs were originally created as a necessary "carve out" to PPS, the LTCH PPS in turn featured what was seen as a necessary carve out: in designing LTCH-PPS, there was concern that LTCHs might discharge patients after a small number of days but still receive the large, lump-sum payments that were intended for longer stays. To address this potential perverse incentive to cycle patients briefly into an LTCH, stays in an LTCH below a certain number of days (the "threshold day") were continued to be paid on the pre-PPS per-diem reimbursement schedule. This created a substantial (approximately \$13,000) jump in Medicare payments at the threshold day, and LTCHs responded by discharging large numbers of patients right after reaching the threshold (Kim et al., 2015; Weaver, Mathews and McGinty, 2015; MedPAC, 2016; Eliason et al., 2018; Einav, Finkelstein and Mahoney, 2018). In Einav, Finkelstein and Mahoney (2018) we explored alternative payment schedules that remove this jump in payments and generate significant savings for Medicare.

In more recent years, CMS has taken at least four distinct measures to try to reduce LTCH spending. In 2007, and again in 2014, CMS established a 3-year moratorium on the certification of new LTCHs or increases in LTCH beds (CMS, 2008, 2014). In 2005, CMS established a policy known as the "25-percent rule" that penalizes LTCHs for admitting more than $25 \%$ of patients in an LTCH from a single hospital, although Congress has delayed the full implementation of the law (42 CFR § 412.534, 2014). In 2011, in order to address incentives for hospitals-within-hospitals to "ping pong" patients between the $\mathrm{ACH}$ and the $\mathrm{LTCH}$, a regulation known as the " 5 percent rule" went into effect, which established that if more than $5 \%$ of patients discharged from an LTCH to a given hospital are later re-admitted to the LTCH, the LTCH will be compensated as if the patient had a single LTCH stay (42 CFR $\S 412.532,2011)$. In 2016, to reduce expenditures and incentivize LTCHs to better target the clini-

\footnotetext{
${ }^{6}$ Calculated from the American Hospital Association data described in the next section.

${ }^{7}$ Profits are defined as EBITA (earnings before interest, taxes, and amortization). Kindred's profits have hovered between $22 \%$ and $29 \%$ of revenue based on 2009 to 2015 company reports. Prior to 2009, Kindred did not separate out their reporting of LTCH profits from the much larger SNF category. Select's profits have ranged between $16 \%$ to $22 \%$ of revenue based on company reports from 2004 to 2015. Kindred's annual reports are available at http://www. annualreports. com/ Company/kindred-healthcare-inc and Select's are available at https: / www. selectmedical.com/investorrelations/for-investors/
} 
cally complex patients they were initially designed to serve, CMS phased in a dual payment structure for LTCHs. Under this new payment structure, LTCHs will be reimbursed under the LTCH PPS only if the patient had an immediately preceding $\mathrm{ACH}$ stay with either (i) 3 or more days in an intensive care unit (ICU) or coronary care unit (CCU), or (ii) mechanical ventilation for at least 96 hours at the LTCH. All other LTCH cases are reimbursed at the lower of the inpatient PPS comparable per diem rate and the total estimated cost incurred by the LTCH to treat the patient (MedPAC, 2017b). Irace (In Progress) studies this reform. Most recently, beginning in 2018, a payment reform went into effect that eliminated the jump in payments at the threshold (80 FR 37990,2017$)$. While it is too soon to be sure, if history is to guide us, the most recent round of reforms will generate new, unintended opportunities for LTCHs to earn profits, and the game of whack-a-mole will continue.

We have dwelled at some length on the institutional and regulatory history of LTCHs because, we believe, it is important for setting our priors and the appropriate null hypothesis: namely, that LTCHs are cost-increasing institutions with no clear benefits to patients. While suggestive, this qualitative history is of course by no means definitive. As noted, existing empirical evidence is limited to crosssectional comparisons of patient outcomes in markets with differential LTCH penetration. We turn now to our data and empirical strategy that will allow us to estimate the impact of LTCHs on average, as well as on particular subsets of patients.

\section{Data and Summary Statistics}

\subsection{Data, Sample, and Variable Definitions}

Our primary data source is the 100\% Medicare Provider and Analysis Review (MedPAR) data from 1998-2014. These data contain claim-level information on all Medicare patient stays at acute care hospitals, LTCHs, SNFs and IRFs. For each stay, the data contain admission and discharge dates, and information on procedures, diagnoses (DRGs), and Medicare payments.

We merge the MedPAR data with three supplementary datasets. The Medicare Annual Beneficiary Summary File provides us with basic patient demographic information, including age, sex, race, and ZIP Code of residence, as well as date of death (if any) through 2014. The beneficiary summary file also includes eligibility and enrollment information, which we use to determine whether a patient is dually eligible for Medicare and Medicaid or enrolled in Medicare Advantage. We exclude all beneficiary-years that have at least one month of enrollment in Medicare Advantage. The Provider of Service (POS) dataset contains annual characteristics for all Medicare-approved providers, which 
allows us to identify each provider's ZIP Code as early as 1984. Finally, we use the American Hospital Association's (AHA) annual survey from 1998-2014 to classify providers as for-profit, non-profit, or government-run, and to obtain provider latitude and longitude, which allow us to calculate distances between facilities.

Our baseline analysis focuses on the entry of the first LTCH into a Hospital Service Area (HSA). HSAs are a standard geographic measure of a health care market. HSAs were originally defined by the National Center for Health Statistics as a collection of contiguous ZIP Codes with at least one hospital where the majority of residents are hospitalized. Since the geographic unit's creation in the early 1990s, HSA boundaries have remained constant regardless of changes to the hospital systems in those regions. There are 3,436 HSAs in the United States, which is similar to the number of counties and roughly ten times the number of Hospital Referral Regions (HRRs), another common geographic unit of analysis. ${ }^{8}$

We use the claim-level MedPAR data to identify whether an LTCH is present in an HSA in each quarter of each year. We define entry as the earliest quarter with a patient admission to an LTCH in that HSA. Appendix A provides more detail on this measure of entry, showing that LTCHs quickly reach steady-state volume after entry; it also shows that our claims-based definition of entry is highly correlated with a measure of entry based on the year of an LTCH's first appearance in the POS file.

In our baseline analysis, our unit of observation is a patient "spell" which we define (following Einav, Finkelstein and Mahoney, 2018) as starting on the date of a patient's admission to an acute care hospital $(\mathrm{ACH})$ and consisting of the set of almost-continuous days with a Medicare payment to an acute care hospital, LTCH, SNF, or IRF. We start the spell with an ACH stay because the vast majority (84\%) of LTCH patients are admitted to an LTCH following their discharge from an ACH. ${ }^{9}$ We end the spell if there are two consecutive days without any Medicare payments to any of these institutions. Note that by this definition, a patient may be readmitted to an $\mathrm{ACH}$ following a stay at a different facility without initiating a new spell. We show in Appendix $C$ that our core results are robust to defining the analysis window as a set amount of time following admission to the ACH.

We analyze a variety of outcomes over the course of a spell. All monetary outcomes are converted to 2014 dollars using the CPI-U. The first set of outcomes is the discharge destination from the ACH. The (mutually exclusive and exhaustive) discharge destinations are to death, to another $\mathrm{ACH}$, to an

\footnotetext{
${ }^{8}$ See www.dartmouthatlas.org/downloads/geography/ziphsahrr98.xls and http://www. dartmouthatlas.org/downloads/methods/geogappdx.pdf for more details on defining HSAs and HRRs.

${ }^{9}$ Most others are admitted directly from the community via a physician referral, although a small number are admitted from other facility-based PAC.
} 
$\mathrm{LTCH}$, to a SNF, or to home/other (where other includes home health care and hospice); Appendix A provides more detail on how we code discharge destinations. We analyze total Medicare payments to and days at various post-discharge facility destinations throughout the spell, as well as total Medicare payments for the spell. ${ }^{10}$ We also analyze total out-of-pocket payments owed for the spell, using the term out-of-pocket payments to refer to payments not covered by Medicare; these payments may be covered by the patient's supplemental insurance plan. Finally, we define indicators for whether the patient has died in the 90-days since the initiating admission to the acute care hospital, and whether the patient has ever been at home in the 90-days since the initiating admission to the acute care hospital. Again, Appendix A provides details.

A potential limitation of our analysis is that the MedPAR data do not include payments to home health or hospice. We have separate data on such payments from 2002-2014. We show in Appendix C that these destinations account for a relatively low share of spell spending (about $10 \%$ combined) and incorporating them into the analysis does not meaningfully impact our findings.

\subsection{LTCH Entry}

Figure 2 shows the distribution of LTCHs across HSAs in the first year that data are available (1984), the first year of our study period (1998), and the last year of our study period (2014). Prior to 1998, 152 HSAs had an LTCH. Over our study period, (1998-2014) an additional 186 HSAs experienced their first entry. The figure also shows that LTCHs tend to be geographically concentrated.

Figure 3 reports the timing of LTCH entry into new HSAs over our study period. First entries occur fairly consistently over the first 12 years of our sample period but drop off in the last few years, presumably due to the moratorium on new facilities. ${ }^{11}$

Table 1 explores characteristics of the hospital markets with LTCHs, separately examining markets that had an LTCH before 1998, experienced their first LTCH entry between 1998 and 2014, and never had an entry. The final column shows the bivariate correlation between an indicator for whether the HSA ever had an LTCH and these characteristics.

Table 1 indicates that LTCHs are more likely to locate in urban and more populated markets, presumably because these markets have enough demand to recover fixed entry costs. In 2014, although only about $10 \%$ of hospital markets had an LTCH, these markets covered $34 \%$ of Medicare beneficia-

\footnotetext{
${ }^{10}$ Our baseline measure includes all Medicare reimbursements except for outlier payments. In Appendix C, we show that including outlier payments makes the point estimates stronger but, because outlier payments are noisy, reduces the precision of our results.

${ }^{11}$ As Figure 3 illustrates, CMS made some exceptions; these are described in more detail in CMS (2008, 2014).
} 
ries. LTCHs tend to be located in markets with a higher rate of $\mathrm{ACH}$ beds per capita, a larger share of for-profit ACHs, and a higher rate of ACH patients discharged to SNF or any PAC (which includes home health care). LTCHs are more likely to enter states that had one of the original LTCHs (defined by presence of an LTCH in 1984) and less likely to enter states with Certificate of Need (CON) laws, which regulate entry. The correlation between entry and these characteristics motivate our event study research design as a complement to prior work that has examined the cross-sectional correlation between outcomes and market-level LTCH penetration (Kahn et al., 2013).

\subsection{Predicted Probability of LTCH Discharge}

While the LTCH setting is high stakes both in terms of Medicare spending and patient health in a given year, many patients are simply not "at risk" of an LTCH discharge and mainly add noise to the estimates. For instance, in 2014, only about 1\% of all hospital patients were discharged to an LTCH. Even in HSAs with LTCHs, only about $2 \%$ of hospital patients were discharged to an LTCH. In order to improve our statistical power, we generate a stay-level measure of the predicted probability of LTCH discharge, and we allow our first stage estimate of the impact of LTCH market entry on LTCH discharge to vary with this ex ante stay-level probability of LTCH discharge. Intuitively, the heterogeneous first stage places more "weight" on patients with a higher ex ante probability of LTCH discharge. We describe our IV approach in more detail in Section 4 below.

Identifying a hospital stay's probability of LTCH discharge (hereafter, $\hat{p}$ ) from the high dimensional set of covariates available in the claims data is a prediction problem well suited to machinelearning methods. We use a regression tree as our prediction algorithm because its emphasis on interactions closely parallels the clinical complexity of LTCH patients, who often have multiple chronic illnesses or comorbidities (Liu et al., 2001; MedPAC, 2016).

We include as predictors demographics and pre-determined health conditions that are plausibly exogenous to the discharge decision. The demographics are the calendar year of the patient admission, patient's age, sex, race, and an indicator for dual enrollment in Medicaid. The health predictors are the ICD-9 diagnoses from the patient's initiating hospital admission. Specifically, we cluster the diagnoses associated with the initiating stay (each stay can have up to 9 distinct diagnoses) into 285 mutually exclusive Clinical Classification Software (CCS) codes (HCUP, 2017). CCS codes have been shown in other settings to be good predictors of health status in Medicare data (Ash et al., 2003; Radley et al., 2008). ${ }^{12}$

\footnotetext{
${ }^{12}$ We exclude procedures in the initial hospital stay from our set of predictors as the propensity to perform certain pro-
} 
As our event study results will confirm, geographic proximity plays a central role in the probability of LTCH discharge. To determine the likelihood of LTCH discharge without geographic constraints, we predict probabilities conditional on having an LTCH in close proximity. To do so, we create a training set consisting of all ACH stays within 5 kilometers of the nearest LTCH, with distance measured as spherical distance based on the provider's latitude and longitude coordinates reported in the AHA provider survey. We train the regression tree on a $10 \%$ sample of these stays using five-fold cross-validation. We then use the estimated prediction model to generate $\hat{p}^{\prime}$ s for all initiating hospital stays (including those further than 5 kilometers away from an LTCH). Thus $\hat{p}$ measures the predicted probability of LTCH discharge if an LTCH were within 5 kilometers of the patient's hospital.

Appendix B provides more detail on both the construction of the prediction algorithm and its output. Because the predictions are generated under the (counterfactual) assumption that all hospital patients are within 5 kilometers of an LTCH, the mean probability of discharge to an LTCH is $2 \%$, rather than $1 \%$ as in the general population. The distribution of $\hat{p}$ is highly right-skewed. This reflects the fact that LTCHs are designed to serve a specific type of clinically complex patients; the vast majority of hospital patients have a very low probability of LTCH discharge, even conditional on having an LTCH in the patient's HSA.

To reduce noise, we construct a "baseline" sample that focuses on all patients with a non-trivial probability of LTCH discharge. Specifically, we drop the 73 million hospital stays (45\%) with a $\hat{p} \leq$ 0.004, which is the "leaf" with the lowest value in the regression tree. This restriction excludes only

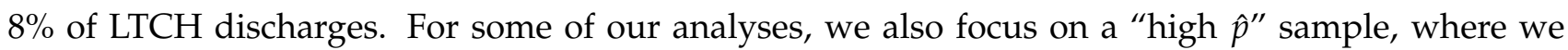
restrict to stays with $\hat{p}>0.15$. This sample keeps $16 \%$ of LTCH discharges.

Table 2 presents summary statistics for the full sample stays, the baseline sample, and the high $\hat{p}$ sub-sample of the baseline sample. Specifically, we report means of patient demographics and our model's "most important" selected health status features, where variable importance is measured by ranking the variables by the additional $R^{2}$ provided at each leaf of the tree. We find that patients with a high probability of LTCH discharge are nearly 10 times as likely to have experienced some sort of respiratory failure and over 10 times as likely to be diagnosed with septicemia (blood poisoning) than the overall acute care population. This is consistent with previous work that finds a high prevalence of patients with sepsis or respiratory failure in LTCHs (MedPAC, 2016; Chen, Vanness and Golestanian, 2011; Koenig et al., 2015). To further assess our model and square our predictions with the existing cedures could be affected by the presence of an LTCH. And, indeed, we provide suggestive evidence of this in Appendix B. 
literature on LTCH patients, the bottom panel of Table 2 reports rates of ICU stays and mechanical ventilation in the initial $\mathrm{ACH}$ stay, two common features of $\mathrm{LTCH}$ patients that have consistently been reported in the literature (Kahn and Iwashyna, 2010; Koenig et al., 2015) but that we excluded from our prediction algorithm due to concerns about potential endogeneity. Encouragingly, we find that over $50 \%$ of high $\hat{p}$ stays spent time in an ICU and over $45 \%$ were on a mechanical ventilator.

\subsection{Summary Statistics}

Table 3 presents means and standard deviations for our primary outcomes for our three event study samples. Column 1 reports results for all acute care admissions. Column 2 shows the baseline sample, which excludes all observations with a $\hat{p} \leq 0.004$, and also restricts attention to the 186 first-entry HSAs and drops quarters following subsequent LTCH entries or exits; this mimics the sample restrictions we use in the baseline event study analyses below. As a result, the event study samples are roughly one seventh the size of the "baseline" sample sizes reported in Table 2, which included the universe of hospitals stays with a $\hat{p} \leq 0.004$. Finally, column 3 shows the high $\hat{p}$ sub-sample of the baseline sample.

A comparison of outcomes in column 2 and column 3 provides a characterization of how patients likely to be discharged from an LTCH differ from other patients. Patients in the high $\hat{p}$ sample require far more intensive, lengthy, and expensive care. High $\hat{p}$ patients have a $13 \%$ probability of being discharged to an LTCH (vs. 1.8\% in the baseline sample), an average spell length of 36 days (compared to 18 in the baseline sample), and average spell Medicare expenditure of over $\$ 42,000$ (vs. roughly $\$ 17,500$ in the baseline sample). ${ }^{13}$ 90-day mortality rates are high in the baseline sample (20\%) and even higher in the high $\hat{p}$ sub-sample (over $40 \%$ ).

\section{Empirical Strategy}

We estimate the effect of LTCH discharge on patient outcomes using variation in LTCH discharges caused by the entry of the first LTCH into a hospital market. Our approach allows outcomes to differ across markets (as suggested by Table 1) but assumes that, in the absence of entry, trends in outcomes would be similar across markets. We examine this assumption by examining trends in outcomes prior to entry.

In our baseline specification, we focus on the entry of the first LTCH in an HSA because this is

\footnotetext{
${ }^{13}$ Because $\hat{p}$ is the probability of LTCH discharge conditional on having one nearby, the true probability of LTCH discharge is lower than the average $\hat{p}$.
} 
where we expect to see the sharpest effects. Specifically, we restrict our sample to the 186 HSAs with a first entry during our 1998-2014 sample period. We exclude the 152 HSAs that, based on the POS annual 1984-1998 files, had an LTCH prior to 1998, and we exclude the over 3,000 HSAs which had no LTCH entry as of 2014. The markets we study are disproportionately large, accounting for $14 \%$ of the Medicare patients and $24 \%$ of LTCH discharges at the end of our sample period. Within the 186 HSAs we study, we truncate the data just before the quarter of second LTCH entry or LTCH exit so that the post-entry results are not contaminated by further shocks to LTCH discharges. Among our 186 HSAs, 24 experience a second entry and 23 an exit. Since the restricted sample is unbalanced, the combination of heterogeneous treatment effects and changes in sample composition might generate spurious time trends in our estimates. We conduct robustness analysis where we restrict the sample to a balanced panel and show that these types of effects are not driving our results.

In order to qualify as an $\mathrm{LTCH}$, a facility must first document that it meets the minimum average length of stay requirement of 25 days for a six-month period (42 CFR § 412.23, 2011). ${ }^{14}$ Most LTCHs therefore begin as an $\mathrm{ACH}$ and are subsequently reclassified as an LTCH. These facilities are neither an LTCH nor an ACH; they are operationally an LTCH but are not reimbursed as such. To address this, we classify a facility that initially opens as an $\mathrm{ACH}$ for a brief period before being deemed an LTCH as an "LTCH in training." Appendix A describes in more detail how we identify them. Our methodology is conservative; as we discuss below, there are likely some LTCHs-in-training that we do not categorize as such.

We define time relative to the quarter of LTCH entry as relative time $(r)$. We consider three distinct periods in relative time: a pre-period $(r<-5)$, a post-period $(r>0)$, and a transition period $(r \in$ $[-5,0])$, in which an LTCH-in-training may have entered prior to the "true" LTCH entry at $r=0$. We draw these distinctions based on patterns in the raw data. In Appendix C, we show the results are robust to alternative plausible time windows for this transition period. The patterns in the raw data also motivate us to allow for separate trends in the outcomes pre and post entry, and to drop from our event study estimates all observations that are associated with the transition period.

Let $i$ index spells, $j$ index HSAs, and $t$ index calendar time (in quarters). Our reduced form speci-

\footnotetext{
${ }^{14}$ In order to retain its LTCH reimbursement rate, a hospital must continue to report a 25-day average length of stay in each cost reporting period.
} 
fication for outcome $y_{i j t}$ takes the form:

$$
y_{i j t}=\alpha \cdot 1\left(r \in P_{\text {post }}\right)+1\left(r \in P_{\text {pre }}\right) f(r)+1\left(r \in P_{\text {post }}\right) g(r)+\gamma_{j}+\tau_{t}+\epsilon_{i j t}
$$

where $\gamma_{j}$ are HSA fixed effects, $\tau_{j}$ are calendar quarter fixed effects, and $f(r)$ and $g(r)$ are linear functions in $r$, normalized such that $f(0)=g(0)=0 .{ }^{15}$ Our parameter of interest $\alpha$ captures the average impact of LTCH entry on patient outcomes. We calculate heteroskedasticity-robust standard errors clustered at the HSA level.

Our identifying assumption is that in the absence of LTCH entry, any trends in the outcome across markets would have been similar. While we cannot test this assumption directly, we present graphical evidence of the time pattern of outcomes prior to LTCH entry that is consistent with the identifying assumption

The parameter $\alpha$ in equation (1) measures the impact of LTCH entry into the market on the outcome. To study the impact of a patient's discharge to an LTCH on outcomes, we estimate instrumental variable (IV) specifications where we use LTCH entry as an instrument for LTCH discharge. Specifically, we estimate the equations

$$
\begin{array}{r}
\text { LTCH }_{i j t}=\alpha \cdot 1\left(r \in P_{\text {post }}\right)+1\left(r \in P_{\text {pre }}\right) f^{L}(r)+1\left(r \in P_{\text {post }}\right) g^{L}(r)+\gamma_{j}^{L}+\tau_{t}^{L}+\epsilon_{i j t}^{L} \\
y_{i j t}=\beta^{y} \cdot \operatorname{LTCH}_{i j t}+1\left(r \in P_{\text {pre }}\right) f^{y}(r)+1\left(r \in P_{\text {post }}\right) g^{y}(r)+\gamma_{j}^{y}+\tau_{t}^{y}+\epsilon_{i j t}^{y}
\end{array}
$$

where the first line shows the "first stage" equation that relates LTCH entry to LTCH discharge, and the second line shows the "second stage" equation that relates LTCH discharge to patient outcome $y_{i j t}$. Both equations include the same controls as the reduced form specification (equation 1), with the parameters allowed to vary across equations. The parameter of interest $\beta^{y}$ can be interpreted as the impact of being discharged to an LTCH on outcome $y_{i j t}$. We calculate heteroskedasticity-robust standard errors clustered at the HSA level.

In the LTCH setting, an additional challenge is that, as discussed in Section 3, the probability of discharge to an LTCH is highly heterogeneous, and near zero for many patients (even if an LTCH

\footnotetext{
${ }^{15}$ Outside of the four-year window around entry, we model $f(r)$ and $g(r)$ as constant in relative time. Specifically, we define

$$
f(r)=\left\{\begin{array}{ll}
a & \text { if } r<16 \\
-b r & \text { if } r \geq-16
\end{array} \text { and } \quad g(r)= \begin{cases}c r & \text { if } r \leq 16 \\
-d & \text { if } r>16\end{cases}\right.
$$

We define these functions in this way because it allows us to focus on LTCH entry inside a four-year window while still preserving observations outside the window to pin down HSA and calendar-time fixed effects.
} 
exists nearby). To improve statistical power, we therefore estimate specifications where we allow the first stage coefficient $(\alpha)$ to vary with $\hat{p}$, the predicted probability of LTCH discharge. Technically, $\hat{p}$ can be interpreted as a compliance propensity score in the spirit of Follmann (2000), which we use to determine heterogeneity in first stage effects.

To allow for a heterogeneous first stage within our event study framework, we divide our baseline sample into five groups indexed by $k=\{1,2,3,4,5\}$. Groups 1 to 3 are quartiles 1 to 3 of the $\hat{p}$ distribution, and groups 4 and 5 are based on splitting the top quartile into two groups $(\hat{p}<0.15$ and $\hat{p}>0.15)$. Appendix Table A1 summarizes these five $\hat{p}$ groups. To account for heterogeneity, we estimate a modified version of our IV specification

$$
\begin{array}{r}
L T C H_{i j t}=\alpha_{k} \cdot 1\left(r \in P_{\text {post }}\right)+1\left(r \in P_{\text {pre }}\right) f_{k}^{L}(r)+1\left(r \in P_{\text {post }}\right) g_{k}^{L}(r)+\gamma_{k r}^{L}+\tau_{k t}^{L}+\epsilon_{i j k t}^{L} \\
y_{i j t}=\beta^{y} \cdot L T C H_{i j t}+1\left(r \in P_{\text {pre }}\right) f_{k}^{y}(r)+1\left(r \in P_{\text {post }}\right) g_{k}^{y}(r)+\gamma_{k j}^{y}+\tau_{k t}^{y}+\epsilon_{i j k t}^{y}
\end{array}
$$

which is identical to equations (2) and (3), except that the first stage coefficient and all of the controls are allowed to vary flexibly by $k$.

We continue to assume that the coefficient of interest $\beta^{y}$ is homogenous across groups, and calculate heteroskedasticity-robust standard errors clustered at the HSA- $\hat{p}$ group level. In the results that follow, we show that, consistent with our homogeneity assumption, our point estimates are very similar, but less precisely estimated, when we restrict the sample to patients with the highest ex ante probability of LTCH discharge $(\hat{p}>0.15)$. In Appendix C, we also show results separately for the other $\hat{p}$ groups, and find that the results are consistent with our homogeneity assumption. In Appendix C, we also show that imposing a first stage specification with a homogenous first stage coefficient $(\alpha)$ results, as expected, in substantially less precise IV estimates.

\section{Results}

\subsection{Reduced Form Graphical Results for High $\hat{p}$ Sample}

Figures 4 to 8 present graphical evidence of the reduced form effects of LTCH entry into the market. In each plot, the horizontal axis shows the relative event time $r$ in quarters and the vertical axis shows the outcome variable. The dots show quarterly averages of the outcome, net of HSA and calendar quarter fixed effects from estimating equation (1). The solid lines show linear trends, $f(r)$ and $g(r)$, which, as shown in equation (1), are separately estimated on the pre- and post-entry periods. For visual effect, 
the dashed line extends the pre-period trend into the transition period. The reduced form effect of LTCH entry on a given outcome, $\alpha$, captures the gap between the linear trends at $r=0$.

We start by examining the effect of LTCH entry into a market on $\hat{p}$, our predicted probably of LTCH discharge. Recall that $\hat{p}$ is constructed using demographics and pre-determined health conditions of patients with ACH stays. If there was an effect of LTCH entry on $\hat{p}$, it would indicate that hospitals are responding endogenously to LTCH entry, for example by changing what patients they admit, which would raise concerns for the interpretation of our empirical results. Reassuringly, Figure 4 shows no evidence of an effect of LTCH entry on $\hat{p}$ in the baseline sample. The estimated reduced form effect of LTCH entry on $\hat{p}$ ( $\alpha$ in equation 1 ) is 0.00050 (standard error $=0.00027$ ), relative to a base of 0.033 pre-entry.

In Figures 5 to 8 we show the reduced form effects of LTCH entry into a market, limited to the high $\hat{p}$ sub-sample of our baseline sample. The first column of Table 4 summarizes the point estimate (and standard error) of the impact of LTCH entry into a market on the outcome ( $\alpha$ in equation 1 ). Figure 5 shows the impact of LTCH entry into a market on the fraction of patients discharged to an LTCH. This will be the first stage in our IV specification. The figure shows that LTCH entry has a sharp impact, raising the probability of discharge to LTCH by 9.2 percentage points (standard error of 0.9), a tripling of the pre-entry probability. The figure also shows evidences of a slight linear trend in LTCH discharges both pre- and post-LTCH entry, which is consistent with LTCHs choosing to enter more rapidly growing markets.

Figure 5 also provides support for our functional form assumptions. The sharp jump at $r=0$ supports our decision to model LTCH entry with a discontinuous jump in the outcome rather than a gradual increase over time. The linear trend fits the data extremely well in the pre-period $(r<-5)$, supporting our identifying assumption that, conditional on controls, the timing of entry is uncorrelated with deviations in the outcome from a linear trend. The linear trends fit well, but with somewhat less precision, in the post period $(r>0)$, perhaps reflecting heterogeneous treatment responses. The decline in discharges to LTCH during the transition period $(r \in[-5,0])$ is consistent with the entry of LTCHs-in-training, which admit patients that would otherwise have gone to an LTCH in the quarters leading up to entry. We see this more directly in Figure 6 discussed below.

Figure 6 shows the effect of LTCH entry into a market on discharges to a set of mutually exclusive and exhaustive non-LTCH discharge destinations. Panel (A) indicates that LTCH entry causes a substantial decline in the fraction of patients discharged to a SNF, suggesting that substitution away 
from SNFs is the primary margin of adjustment. Panel (B) shows a smaller, but non-negligible, decline in discharges to home/other, suggesting more modest substitution on this margin. Panel (C) shows a sharp increase in discharges to LTCHs-in-training during the transition period only, which is what we would expect given the institutional requirements to qualify as an LTCH. Panel (D) also shows some evidence of an increase in discharges to ACHs during the transition period only, which may reflect discharges to LTCHs-in-training that we did not classify using our algorithm. Panel (E) shows no evidence of a change in the probability of discharge to death (i.e. in-hospital death) following the entry of an LTCH.

Figure 7 shows the effect of LTCH entry into a market on total spell days and total Medicare spending during the spell. Recall that the main effect of LTCH entry was substitution from SNFs to LTCHs. Panel (A) shows little effect on total spell days, suggesting that the marginal patients have similar lengths of stay at SNFs and LTCHs. Panel (B), on the other hand, shows that LTCH entry into a market leads to a fairly large increase in total Medicare spending, which is consistent with LTCHs receiving larger daily reimbursements than SNFs. ${ }^{16}$

Finally, Figure 8 shows the impact LTCH entry into a market on three measures of patient well being: total out-of-pocket spell spending, the probability the patient is ever back home within 90 days after the initial hospital admission, and 90-day mortality (also measured from the date of the initial hospital admission). The graphical results suggest a clear increase in out-of-pocket spending. There is some suggestive evidence of a slight decrease in the probability of being at home at any point within 90 days. Despite the high 90 -day mortality rate (44\% in the high $\hat{p}$ sample), the 90 -day mortality plot shows no evidence of any obvious pattern, and is quite noisy.

\subsection{Estimates}

Columns 2 and 3 of Table 4 show the IV estimates of the effect of discharge to an LTCH. Column 2 shows point estimates and standard errors in the high $\hat{p}$ sample, and column 3 shows the average impact of discharge to LTCH on patient outcomes for the whole baseline sample, allowing for a heterogeneous first stage to improve power. In the baseline sample, the share of patients discharged to LTCH increases from $0.5 \%$ in $r=-6$ (just before the transition period) to $2.4 \%$ in $r=2$ (just after the transition period). This implies that our effects are identified off the $80 \%$ of patients in the baseline sample who are marginal to LTCH entry, but are silent on the effects for the $20 \%$ of patients who

\footnotetext{
${ }^{16}$ Appendix Figure A1 provides a more detailed perspective, showing the effect of LTCH entry on days and spending separately by type of facility (LTCH, SNF, initiating ACH).
} 
would have counterfactually been discharged to LTCHs in the absence of LTCH entry. Consistent with our assumption that the impact of discharge to LTCH on patient outcomes is constant across patients with different $\hat{p}$ 's, the IV estimates in the high $\hat{p}$ sub-sample and the baseline sample are usually quantitatively very similar, and are never statistically distinguishable. We therefore focus our discussion below on the IV results for the full baseline sample (column 3). ${ }^{17}$

The top panel of Table 4 show IV estimates of the effect of LTCH discharge on non-LTCH discharge locations. The results indicate that about four-fifths of patients discharged to an LTCH would have otherwise been discharged to a SNF; the remaining one-fifth would have otherwise been discharged to home without home care or other (which includes home with home health care, hospice, and other facility care). More specifically, we estimate that each patient discharged to an LTCH reduced the probability of discharge to a SNF by 0.791 (standard error of 0.075 ) and to home/other by 0.236 (standard error of 0.073 ).

A limitation to our baseline data is that we cannot see any finer granularity on the discharge destination of "home/other." However, for a subset of our study period (2002-2014), additional data allow us to further decompose this discharge destination; Appendix $\mathrm{C}$ shows the results. We find that about half of the impact on the discharge destination "home/other' reflects a decline in discharges home without home health care, and the rest stems from a decline in discharges to a residual "other" category; there is no evidence of any decline in discharges to home with home health care or in discharges to hospice.

The next two panels of Table 4 show results for spell days and spell spending. Focusing again on the IV estimates for the baseline sample in column 3, our results indicate that discharge to an LTCH increases total spell days by a statistically insignificant 6.6 days (standard error of 5.1); days in both SNF and the initiating ACH decrease, while days in LTCH increase. The 8.6 day average decline in length of stay at the initiating ACH is consistent with the claim that LTCHs in some cases provide care to patients that they could not receive at other forms of institutional PAC (NALTH, 2018); when the patient is not discharged to an LTCH she spends, on average, considerably longer in the ACH. Given that ACHs are paid a lump sum per patient that is (largely) independent of length of stay, the decline in length of ACH stay associated with LTCH discharge suggests that not only LTCHs, but also ACHs,

\footnotetext{
${ }^{17}$ For completeness, Appendix Table A2 presents first stage and IV estimates for the five $\hat{p}$ groups. Consistent with the interpretation of $\hat{p}$ as an estimated compliance propensity score, the first stage increases monotonically with $\hat{p}$ group. Although the results are, as expected, less precise in the lower $\hat{p}$ bins, the broad similarity in estimates across groups is consistent with our assumption that the impact of discharge to an LTCH on patient outcomes is the same across patients in different groups.
} 
may benefit financially from discharge to LTCH. We return to this point in the conclusion.

Discharge to an LTCH increases total spell spending by $\$ 29,583$ (standard error of $\$ 4,810$ ). This represents about $169 \%$ increase in total spell spending relative to the average spell spending of $\$ 17,519$ (see Table 3). The increase in spending reflects a $\$ 34,569$ increase in LTCH spending, that is only slightly offset by a decline in SNF spending. ${ }^{18}$

The final panel of Table 4 shows results for three measures of patient welfare. There is no evidence of discharge to LTCH improving patient welfare on any of these measures. Discharge to LTCH is associated with increased amounts owed out of pocket of \$2,420 (standard error \$640). ${ }^{19}$ There is no evidence that LTCHs increase the probability of being at home at any point in the 90 days post admission to the initial acute hospital admission; indeed the point estimates suggests a statistically insignificant decline (consistent with the statistically insignificant increase in institutional days and in mortality). The final measure of patient welfare we look at is 90-day mortality; this is quite high in our baseline sample (20\%; see Table 3). However, we find no evidence that discharge to LTCH reduces mortality. Indeed, the point estimate suggests that discharge to LTCH is associated with a statistically insignificant increase in 90-day mortality of 10.1 percentage points; the $95 \%$ confidence interval allows us to rule out mortality declines greater than 2.6 percentage points.

Appendix Table A3 explores these mortality results in more detail, examining results over different horizons from 30 days to a year; at all these horizons we are unable to reject the null hypothesis of no impact of discharge to LTCH on mortality. We are unable to measure other potential non-mortality health benefits or non-health utility benefits from an LTCH stay. Our estimates in Table 4, however, indicate that any such unmeasured benefits would need to be valued at over $\$ 32,003$ per LTCH stay (increased Medicare spending of $\$ 29,583$ plus increased out-of-pocket spending of $\$ 2,420$ ) in order to cover the incremental healthcare spending associated with LTCH discharge. With an increase in length of stay of 28.9 days on average, this would require LTCHs to provide an incremental \$1,107 in daily value.

\footnotetext{
${ }^{18}$ We omit from the table two other possible sources of institutional days and spending: days and spending at an LTCHin-training and data at another ACH. The effects are substantively small and statistically insignificant: LTCH in training utilization increases by 0.16 days (standard error of 0.09 ) and $\$ 113$ in spending (standard error of 50), and ACH utilization increases by 0.71 days (standard error of 1.29) and $\$ 629$ (standard error of $\$ 1,930$ ).

${ }^{19}$ LTCH stays are covered under inpatient cost-sharing. In practice, 95\% of LTCH stays involve no deductible. However, patients are exposed to per-day coinsurance that applies starting on day 61 of the benefit period. In 2014, LTCH stays resulted in an average $\$ 2,250$ in coinsurance owed out-of-pocket. By contrast, the first 20 days in a SNF have no patient cost-sharing.
} 


\subsection{Heterogeneous impacts and robustness}

We examine potential heterogeneity in the impact of LTCHs on a number of dimensions. Table 5 summarizes these results; Appendix Tables A4 and A5 provide additional details on the heterogeneity results while Appendix Figures A2 and A3 show the first stage figures for the high $\hat{p}$ samples for each cut of the data.

Panel A of Table 5 explores whether the impact of LTCHs differs for patients who - under the 2016 payment reform described in Section 2 - will still be reimbursed under LTCH reimbursement rules. As discussed, this requires that the patient's immediately preceding $\mathrm{ACH}$ stay have either 3 or more days in an intensive care unit (ICU) or coronary care unit (CCU), as analyzed in Panel A, or mechanical ventilation for at least 96 hours at the LTCH, as proxied in Panel B by an indicator for whether or not the patient was ventilated at the initiating $\mathrm{ACH}$. Consistent with the idea that these reforms were designed to exclude patients for whom other forms of PAC are a reasonable substitute, patients whose reimbursement at LTCH rates was subsequently excluded under the 2016 reform show more substitution away from SNF in response to LTCH discharge. However, there is no evidence that those patients who would still qualify for LTCH reimbursement under the new policy experience lower spending effects or greater patient welfare from LTCH discharge; indeed, if anything the point estimates are suggestive of the opposite, although we are unable to reject the null hypothesis that effects are the same across groups. Appendix Table A4 considers a more stringent regulation, originally proposed by CMS but weakened when enacted into law by Congress: that patients must stay more than 8 days (rather than 3) in an ICU or CCU in order to be reimbursed using the LTCH rates (MedPAC, 2014). The results for this split of the data are again quite similar.

Our analysis of LTCH entries does not capture the effects for infra-marginal patients who would have traveled outside of their MSA to receive care in an LTCH if there had not be an LTCH entry in their MSA. To shed some light on the effects for these type of patients, Panel C of Table 5 compares impacts of LTCHs across markets that had a higher or lower pre-entry LTCH discharge share. Specifically, we compare results for those markets below and above the median share of patients discharged to LTCHs in relative quarter $r=-6$; where the average share was $0.27 \%$ and $0.89 \%$ respectively. The results seem mostly similar between the two groups, with some evidence that LTCHs that enter higher pre-entry LTCH discharge markets may have worse impacts on patients (who are less likely to go home and more likely to die within 90 days). In Panel D, we also compared results across for-profit and non-profit LTCHs but see no evidence of differential impacts. 
We also explore the robustness of our findings to a number of alternative specifications. Appendix C presents the results, which are reassuring. Our baseline analysis allowed for a transition period from relative year -5 to 0 , in which an LTCH-in-training may have entered prior to the "true" LTCH entry at $r=0$. We explore alternative transition periods, both shorter (-2 to 0$)$ and longer ( -5 to 5$)$. Our baseline analysis is limited to the 186 markets where LTCHs entered for the first time during our study period (1998 to 2014). We show the results are robust to including the 152 markets with pre-existing LTCHs as controls. We also show that our results are robust to alternative geographic definitions of healthcare markets (specifically, ZIP Code or county rather than HSA), and to including entries of additional LTCHs after the first in the market. We also show the results are robust to a balanced panel, to excluding Medicaid dual eligibles from the analysis, to defining a spell as one-year post admission to the $\mathrm{ACH}$, and to including additional data on home health and hospice payments.

\subsection{Implications}

We briefly explored the implications of our estimates for aggregate Medicare spending and for the much-studied geographic variation in Medicare spending. In 2014, Medicare spending on LTCHs was $\$ 5.4$ billion (MedPAC, 2016). Our estimates in Table 4 indicate that about $85 \%$ of LTCH spending (i.e. $\$ 29,583 / \$ 34,569)$ represents incremental spending. This suggests that the elimination of LTCHs would reduce Medicare spending by about $\$ 4.6$ billion per year, with no measurable adverse impact on patient welfare.

Relatedly, we can use our estimates to ask what share of the large, and much-discussed, geographic variation in Medicare spending would be eliminated if Medicare patients were no longer sent to LTCHs. The finding of substantial differences across areas in Medicare spending per enrollee - without correspondingly better health outcomes - has been widely-touted as suggestive of waste and inefficiency in the U.S. healthcare system (e.g., CBO, 2008; Gawande, 2009; Skinner, 2011). As noted earlier, an influential report by the Institute of Medicine estimated that almost three-quarters of the unexplained geographic variation in Medicare spending could be explained by spending on PAC (IOM, 2013). This analysis, however, assumed that there would be no behavioral response to the removal of PAC. We can use our estimates of the behavioral response to LTCHs - i.e., how much of LTCH spending is incremental as opposed to substitution from SNFs - to ask how much geographic variation in spending would be reduced if LTCHs were removed. We closely replicate the IOM (2013) finding - specifically we find that eliminating PAC would reduce residual variance by 69\% (compared 
to their $73 \%$ reported estimate). We find that eliminating LTCHs - which are only $1 \%$ of Medicare spending - would remove $13 \%$ of the residual variance, in the absence of a behavioral response, and about $10 \%$ given the substitution to SNFs. Consistent with the Dartmouth Atlas interpretation of the geographic variation as evidence of waste and inefficiency, our estimates suggest that this reduction in geographic variation would come without any adverse effects on patient well-being.

\section{Conclusion}

LTCHs were originally intended as a small administrative carve-out to the new inpatient prospective payment system designed in 1982. Inadvertently, however, their designation created a regulatory loophole for post-acute care facilities to receive substantially higher reimbursements. Over the ensuing decades, CMS has endeavored, through a series of legislative and regulatory reforms, to try to close this loophole. Its continued attempts suggest that is has not yet been successful, and raise real questions about whether incremental reforms will ever achieve their goals.

Our empirical estimates suggest that by simply eliminating the administratively-created concept of LTCHs as an institution with its own reimbursement schedule - and reimbursing them instead like SNFs - Medicare could save $\$ 4.6$ billion per year with no harm to patients. Moreover, despite accounting for only about $1 \%$ of Medicare spending, we estimate that eliminating LTCHs would reduce $10 \%$ of the unexplained geographic variation in Medicare spending. As is the case with any counterfactual, one must always be careful to not go too far out of sample. From this perspective, a strength of our analysis is that it provides the rare opportunity to study a non-marginal change: the entry of a new healthcare institution into a healthcare market.

Nonetheless, there are (at least) two potential caveats to keep in mind in generalizing from our estimates to the impact of changing LTCH reimbursement to that of SNFs. First, we study the impact of LTCHs at the time of their creation. It is of course possible that the longer-run impacts of LTCHs on either spending or patient well-being are different (with unknown sign) from what we estimate here, although the graphical analysis we present in Figures 5 through 8 do not suggest any obvious differences in the first four years of the LTCH's existence.

Second, our estimates of the impact of LTCHs are not based on all LTCH patients. Our baseline sample excludes the approximately $15 \%$ of LTCH patients who are not admitted from an ACH and the approximately $8 \%$ of LTCH admissions from an ACH that involve patients whose ex-ante probability of discharge to an LTCH is less than $0.4 \%$. Our estimates also do not speak to the effects for the 
$20 \%$ of patients who would have counterfactually been discharged to LTCHs in the absence of LTCH entry into their HSA. In this respect, we find it reassuring that we were unable to detect any evidence of heterogeneous impacts of LTCHs across patients, markets or outcomes; in particular, we find no evidence of differential effects across markets with different shares of patients who would have counterfactually been discharged to LTCHs in the absence of LTCH entry into their HSA, for-profit and non-profit LTCHs, and patients whose LTCH stays are or are not eligible for LTCH reimbursement rates under the 2016 dual payment reform (MedPAC, 2017b).

More broadly, our paper suggests that successfully reducing waste in the healthcare sector may involve more forensic investigation than health economists and health policy experts typically engage in. Our reading of past efforts at "reducing waste" in U.S. healthcare is that they have emphasized broad-based reforms to delivery models, often motivated by economic theory - price regulation and certificate of need laws in the 1970s (Joskow, 1981), Prospective Payment and managed care systems in the 1980s and 1990s (Newhouse, 1996), and most recently, the move to Alternative Payment Models such as Accountable Care Organizations and bundled payments (Berwick, 2011). These efforts have consistently failed to fulfill the high expectations set for them, and have often produced unintended, negative consequences. By contrast, our analysis of a specific institution that appears to be wasteful is closer to the approach Duflo (2017) advocates: "the economist as plumber ... she installs the machine in the real world, carefully watches what happens, and then tinkers as needed." Our analysis of the case of LTCHs provides an illustration of how the health economist might fruitfully transform into the health plumber.

We finish with a note of caution: while changing the reimbursement of LTCHs might be viewed as tinkering relative to the broad-based reform discussed above, there is no reason to expect that such tinkering will be politically easy. The $\$ 4.6$ billion of incremental spending generated by LTCHs every year may look like "waste" to the health economist, but to the (largely for-profit) LTCH industry it might more accurately be referred to as "rents." Acute Care Hospitals (ACHs) likely also benefit from the presence of LTCHs since, we found, discharges to LTCHs reduce length of stay at the initiating $\mathrm{ACH}$, who bear the incremental costs of additional days. This suggests a large financial incentive on the part of LTCHs as well as ACHs to preclude major regulatory changes, and may help explain their continued survival. 


\section{References}

42 CFR § 412.23. 2011. "Excluded hospitals: Classifications." Code of Federal Regulations.

42 CFR § 412.532. 2011. "Special payment provisions for patients who are transferred to onsite providers and readmitted to a long-term care hospital." Code of Federal Regulations.

42 CFR § 412.534. 2014. "Special payment provisions for long-term care hospitals-within-hospitals and satellites of long-term care hospitals, effective for discharges occurring in cost reporting periods beginning on or before September 30, 2016." Code of Federal Regulations.

80 FR 37990 . 2017. “A Rule by the Centers for Medicare \& Medicaid Services." Federal Regester.

AHPA. 2003-2011. “National Directory of Health Planning, Policy and Regulatory Agencies." American Health Planning Association.

Anderson, Gerard F, Peter S Hussey, Bianca K Frogner, and Hugh R Waters. 2005. "Health spending in the United States and the rest of the industrialized world." Health Affairs, 24(4): 903-914.

Ash, Arlene S, Michael A Posner, Jeanne Speckman, Shakira Franco, Andrew C Yacht, and Lindsey Bramwell. 2003. "Using claims data to examine mortality trends following hospitalization for heart attack in Medicare." Health Services Research, 38(5): 1253-1262.

Barnett, Michael L, Andrew R Olenski, and Anupam B Jena. 2017. "Opioid-prescribing patterns of emergency physicians and risk of long-term use." New England Journal of Medicine, 376(7): 663-673.

BEA. 2016. "Download NIPA Tables: Government Current Receipts and Expenditures (Section 3)."

Berwick, Donald M. 2011. "Launching accountable care organizations-the proposed rule for the Medicare Shared Savings Program." New England Journal of Medicine, 364(16): e32.

Boards of Trustees for Medicare. 2002. "The 2002 Annual Report of the Boards of Trustees of the Federal Hospital Insurance Trust Fund and the Federal Supplementary Medical Insurance Trust Fund."

Boards of Trustees for Medicare. 2014. "The 2014 Annual Report of the Boards of Trustees of the Federal Hospital Insurance Trust Fund and the Federal Supplementary Medical Insurance Trust Fund."

Boards of Trustees for Medicare. 2015. "The 2015 Annual Report of the Boards of Trustees of the Federal Hospital Insurance Trust Fund and the Federal Supplementary Medical Insurance Trust Fund."

Buntin, Melinda Beeuwkes. 2007. "Access to postacute rehabilitation." Archives of physical medicine and rehabilitation, 88(11): 1488-1493.

CBO. 2008. "Geographic Variation in Health Care Spending." Congressional Budget Office.

CBO. 2016. “Updated Budget Projections: 2016 to 2026." Congressional Budget Office.

Chandra, Amitabh, and Jonathan Skinner. 2012. "Technology growth and expenditure growth in health care." Journal of Economic Literature, 50(3): 645-80.

Chen, Han-Yang, David J Vanness, and Ellie Golestanian. 2011. "A simplified score for transfer of patients requiring mechanical ventilation to a long-term care hospital." American Journal of Critical Care, 20(6): e122-e130. 
Clemens, Jeffrey, and Joshua D Gottlieb. 2014. "Do physicians' financial incentives affect medical treatment and patient health?" American Economic Review, 104(4): 1320-49.

CMS. 2008. "Moratorium on Classification of Long-Term Care Hospitals (LTCH) or Satellites/Increase in Certified LTCH Beds." Centers for Medicare \& Medicaid Services.

CMS. 2014. “Long-term Care Hospital (LTCH) Moratorium - Preliminary Instructions." Centers for Medicare \& Medicaid Services.

Curto, Vilsa, Liran Einav, Amy Finkelstein, Jonathan D Levin, and Jay Bhattacharya. 2019. "Healthcare spending and utilization in public and private Medicare." American Economic Journal: Applied Economics, 11(2): 302-32.

Cutler, David. 2010. "How Health Care Reform Must Bend The Cost Curve." Health Affairs, 29(6): 1131-1135.

Cutler, David M. 1995. "The Incidence of Adverse Medical Outcomes Under Prospective Payment." Econometrica, 63(1): 29-50.

Doyle, Joseph J, John A Graves, and Jonathan Gruber. 2017. “Uncovering waste in US healthcare: Evidence from ambulance referral patterns." Journal of health economics, 54: 25-39.

Doyle, Joseph, John Graves, and Jonathan Gruber. 2015. “In hospital care saves money - and lives." Boston Globe.

Duflo, Esther. 2017. "Richard T. Ely Lecture: The Economist as Plumber." American Economic Review, 107(5): 1-26.

Einav, Liran, Amy Finkelstein, and Neale Mahoney. 2018. "Provider incentives and healthcare costs: Evidence from long-term care hospitals." Econometrica, 86(6): 2161-2219.

Eliason, Paul J, Paul LE Grieco, Ryan C McDevitt, and James W Roberts. 2018. "Strategic Patient Discharge: The Case of Long-Term Care Hospitals." American Economic Review, 108(11): 3232-65.

Follmann, Dean A. 2000. “On the effect of treatment among would-be treatment compliers: An analysis of the multiple risk factor intervention trial." Journal of the American Statistical Association, 95(452): 1101-1109.

Gawande, Atul. 2009. "The Cost Conundrum: What a Texas Town Can Teach Us about Health Care." New Yorker.

HCUP. 2017. "Clinical Classifications Software (CCS) for ICD-9-CM.” Healthcare Cost and Utilization Project.

Ho, Kate, and Ariel Pakes. 2014. "Hospital choices, hospital prices, and financial incentives to physicians." American Economic Review, 104(12): 3841-84.

IOM. 2013. Variation in health care spending: target decision making, not geography. National Academies Press.

Irace, Mariano. In Progress. “The Impact of Payment Reform on the Behavior of Long-Term Care Hospitals."

Joskow, Paul L. 1981. Controlling hospital costs: The role of government regulation. 
Kahn, Jeremy M, and Theodore J Iwashyna. 2010. "Accuracy of the discharge destination field in administrative data for identifying transfer to a long-term acute care hospital." BMC research notes, 3(1): 205.

Kahn, Jeremy M, Rachel M Werner, Guy David, Thomas $R$ Ten Have, Nicole $M$ Benson, and David A Asch. 2013. "Effectiveness of long-term acute care hospitalization in elderly patients with chronic critical illness." Medical care, 51(1): 4.

Kim, Yan S, Eric C Kleerup, Patricia A Ganz, Ninez A Ponce, Karl A Lorenz, and Jack Needleman. 2015. "Medicare payment policy creates incentives for long-term care hospitals to time discharges for maximum reimbursement." Health Affairs, 34(6): 907-915.

Koenig, Lane, Berna Demiralp, Josh Saavoss, and Qian Zhang. 2015. “The Role of Long-term Acute Care Hospitals in Treating the Critically Ill and Medically Complex: An Analysis of Nonventilator Patients." Medical care, 53(7): 582.

Liu, Korbin, Cristina Baseggio, Douglas Wissoker, Stephanie Maxwell, Jennifer Haley, and Sharon Long. 2001. "Long-term care hospitals under Medicare: facility-level characteristics." Health care financing review, 23(2): 1.

McGinnis, J Michael, Leigh Stuckhardt, Robert Saunders, Mark Smith, et al. 2013. Best care at lower cost: the path to continuously learning health care in America. National Academies Press.

MedPAC. 2014. “Chapter 11: Long-term Care Hospital Services.” Medicare Payment Advisory Commission.

MedPAC. 2015a. "A Data Book: Health Care Spending and the Medicare Program." Medicare Payment Advisory Commission.

MedPAC. 2015b. “Report to the Congress.” Medicare Payment Advisory Commission.

MedPAC. 2016. "Chapter 10: Long-term Care Hospital Services." Medicare Payment Advisory Commission.

MedPAC. 2017a. "Long-Term Care Hospitals Payment System." Medicare Payment Advisory Commission.

MedPAC. 2017b. "Report to the Congress: Medicare Payment Policy, Chapter 11: Long-term care hospital services." Medicare Payment Advisory Commission.

MedPAC. 2018. "Report to the Congress: Medicare Payment Policy, Chapter 11: Long-term care hospital services." Medicare Payment Advisory Commission.

Molitor, David. 2018. "The evolution of physician practice styles: evidence from cardiologist migration." American Economic Journal: Economic Policy, 10(1): 326-56.

NALTH. 2018. "A Response to the National Bureau of Economics Research Working Paper, "LongTerm Care Hospitals: A Case Study in Waste" A Response to National Bureau of Economics Research Working Paper, "Long-Term Care Hospitals: A Case Study in Waste"." National Association of Long Term Hospitals.

Newhouse, Joseph P. 1996. "Reimbursing health plans and health providers: efficiency in production versus selection." Journal of economic literature, 34(3): 1236-1263.

OECD. 2017. "Health at a Glance 2017: OECD Indicators." OECD. 
Office of Inspector General. 2013. "Early Alert Memorandum Report: Co-Located Long-Term Care Hospitals Remain Unidentified, Resulting in Potential Overpayments." Department of Health and Human Services.

Orszag, Peter R. 2009. "Heath Costs are the Real Deficit Threat." The Wall Street Journal.

Ottenbacher, Kenneth J, and James E Graham. 2007. "The state-of-the-science: access to postacute care rehabilitation services. A review." Archives of Physical Medicine and Rehabilitation, 88(11): 15131521.

Papanicolas, Irene, Liana R Woskie, and Ashish K Jha. 2018. "Health care spending in the United States and other high-income countries." Journal of the American Medical Association, 319(10): 10241039.

Radley, David C, Daniel J Gottlieb, Elliot S Fisher, and Anna NA Tosteson. 2008. "Comorbidity risk-adjustment strategies are comparable among persons with hip fracture." Journal of clinical epidemiology, 61(6): 580-587.

Skinner, Jonathan. 2011. "Causes and consequences of regional variations in health care." In Handbook of health economics. Vol. 2, 45-93. Elsevier.

Weaver, Christopher, AW Mathews, and Tom McGinty. 2015. "Hospital discharges rise at lucrative times." Wall Street Journal. 
Figure 1: LTCH Facilities over Time

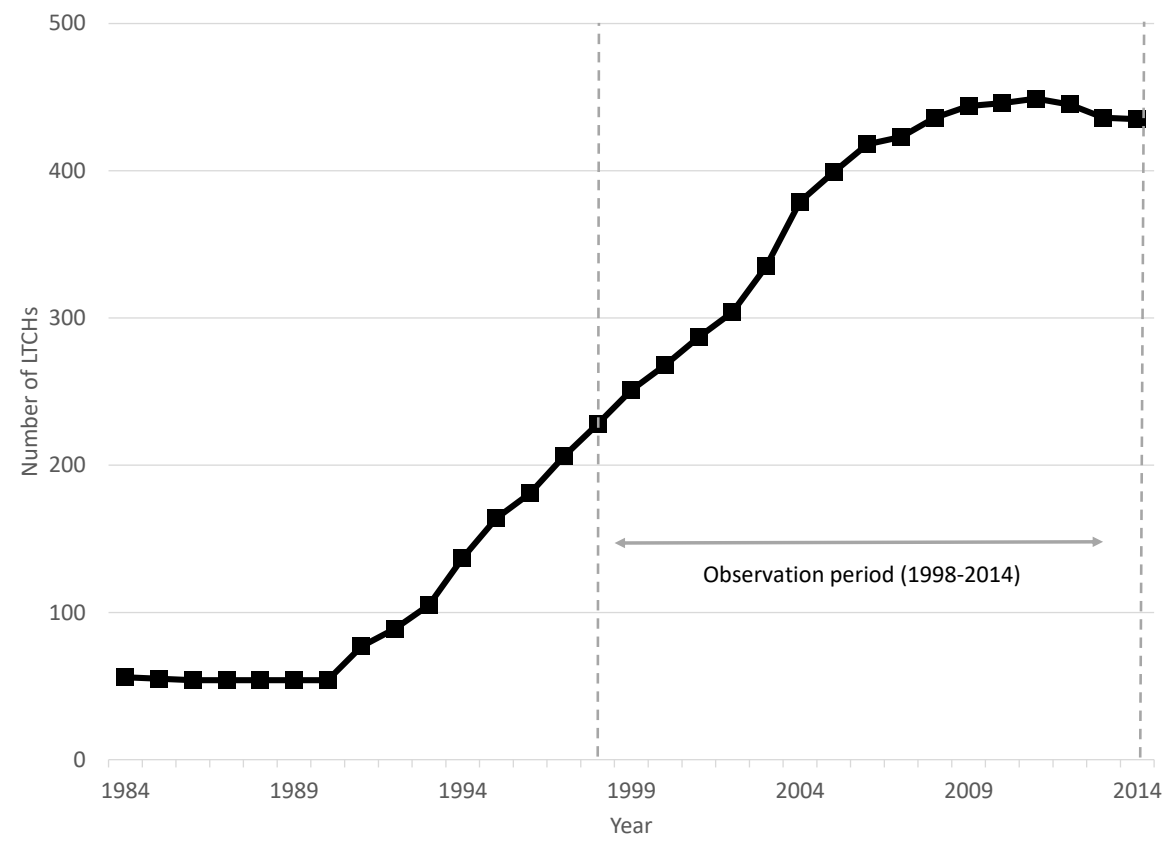

Note: Data from the Provider of Service (POS) File from 1984-1998 and the MedPAR data from 1998-2014. Figure only includes LTCHs that appear in the MedPAR data. 
Figure 2: LTCH Concentration over Time

(A) 1984

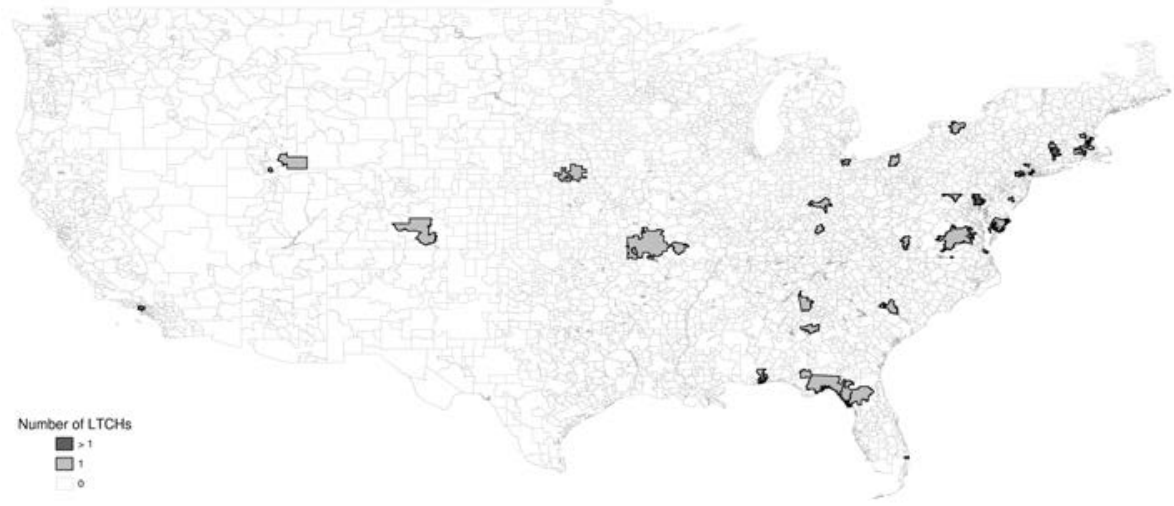

(B) 1998

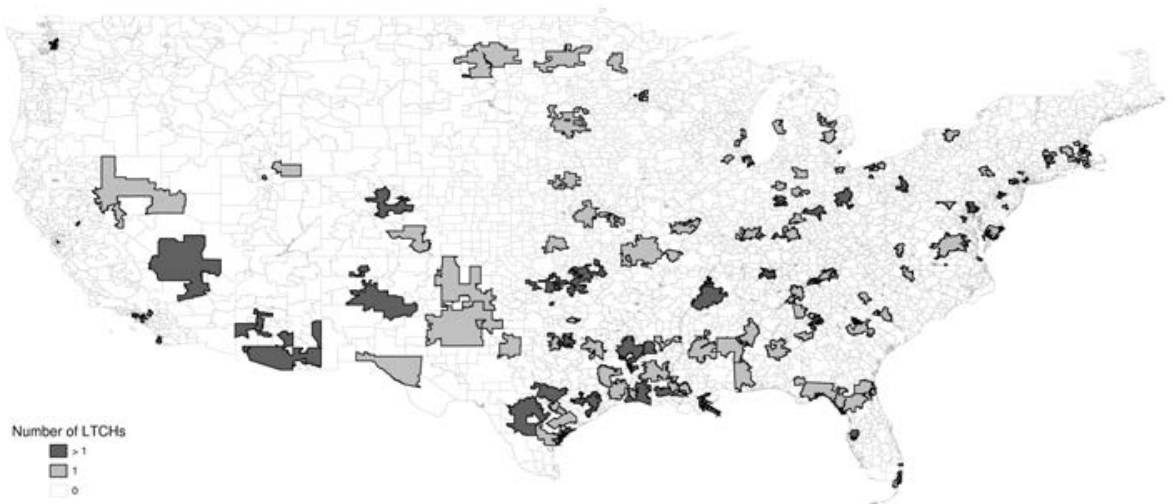

(C) 2014

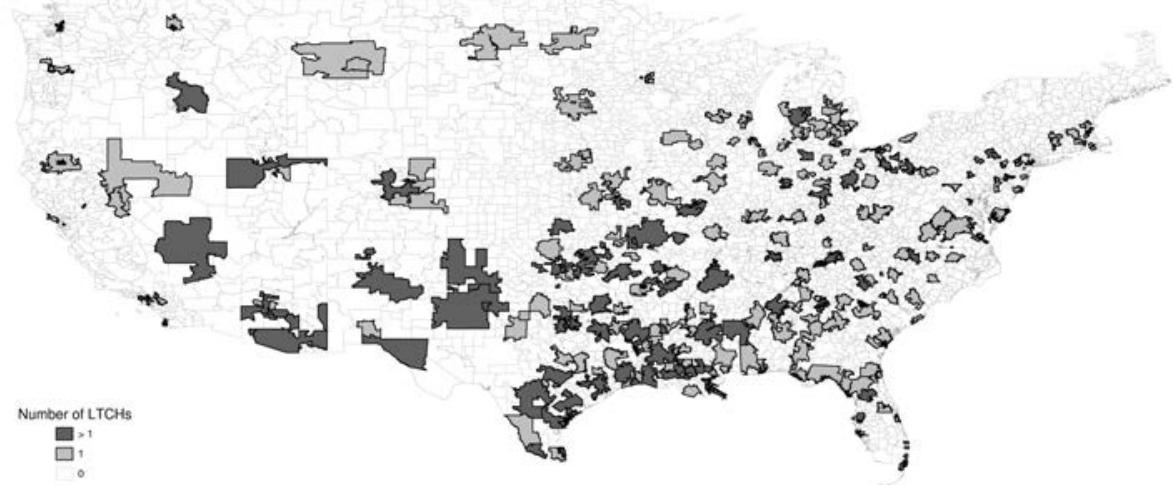

Note: Maps illustrate the number of LTCHs present in each HSA in 1984, 1998, and 2014, according to the 1984-2016 POS File and the 1998-2014 MedPAR data. White space indicates HSAs with no LTCHs. 
Figure 3: LTCH Entry Quarters

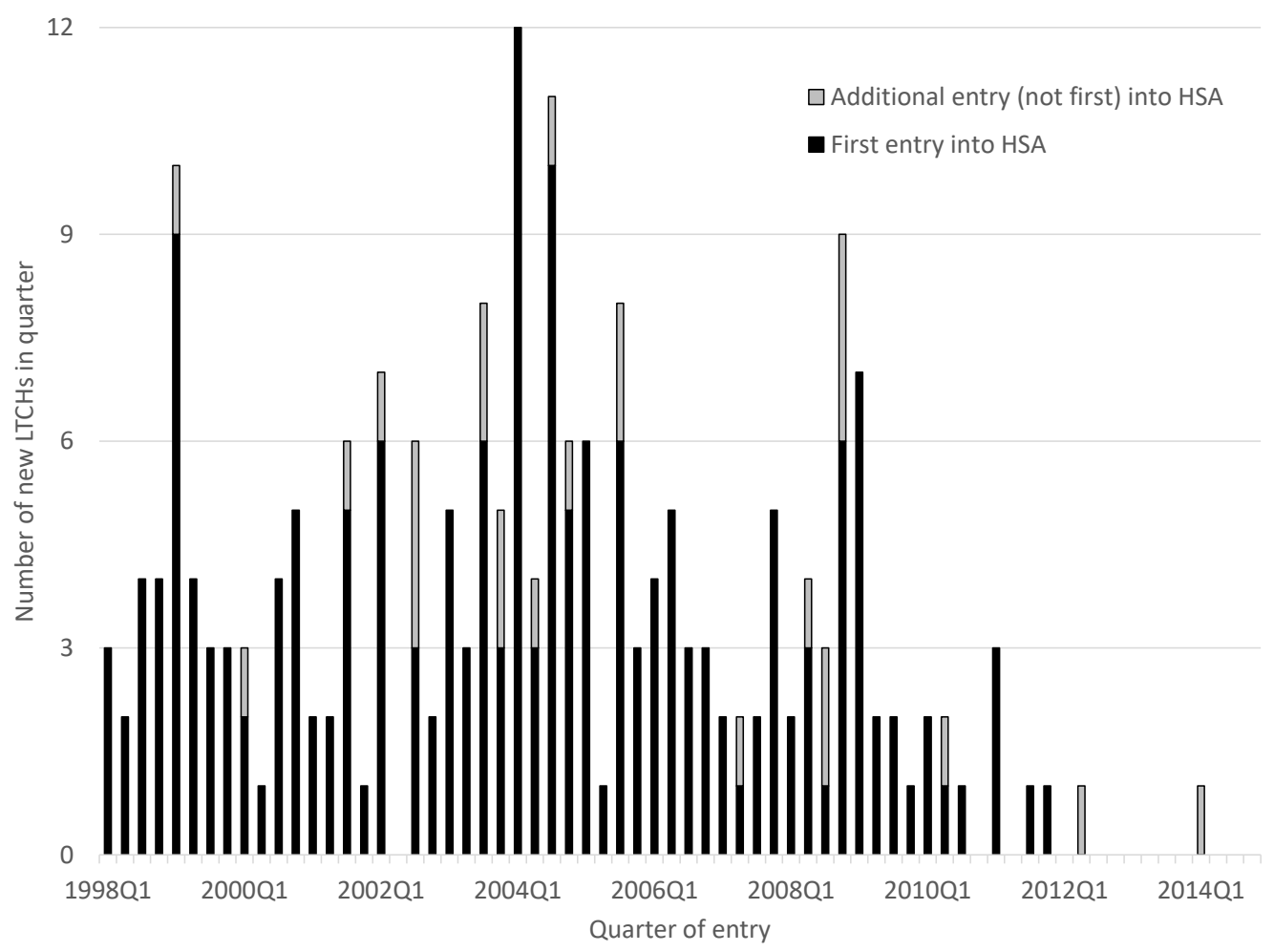

Note: Histogram of LTCH entry to an HSA. First entries are defined as the first entry observed in our data (1998-2014), and thus excludes HSAs that had an LTCH at the start of our data period. 
Figure 4: Predicted Probability of LTCH Discharge

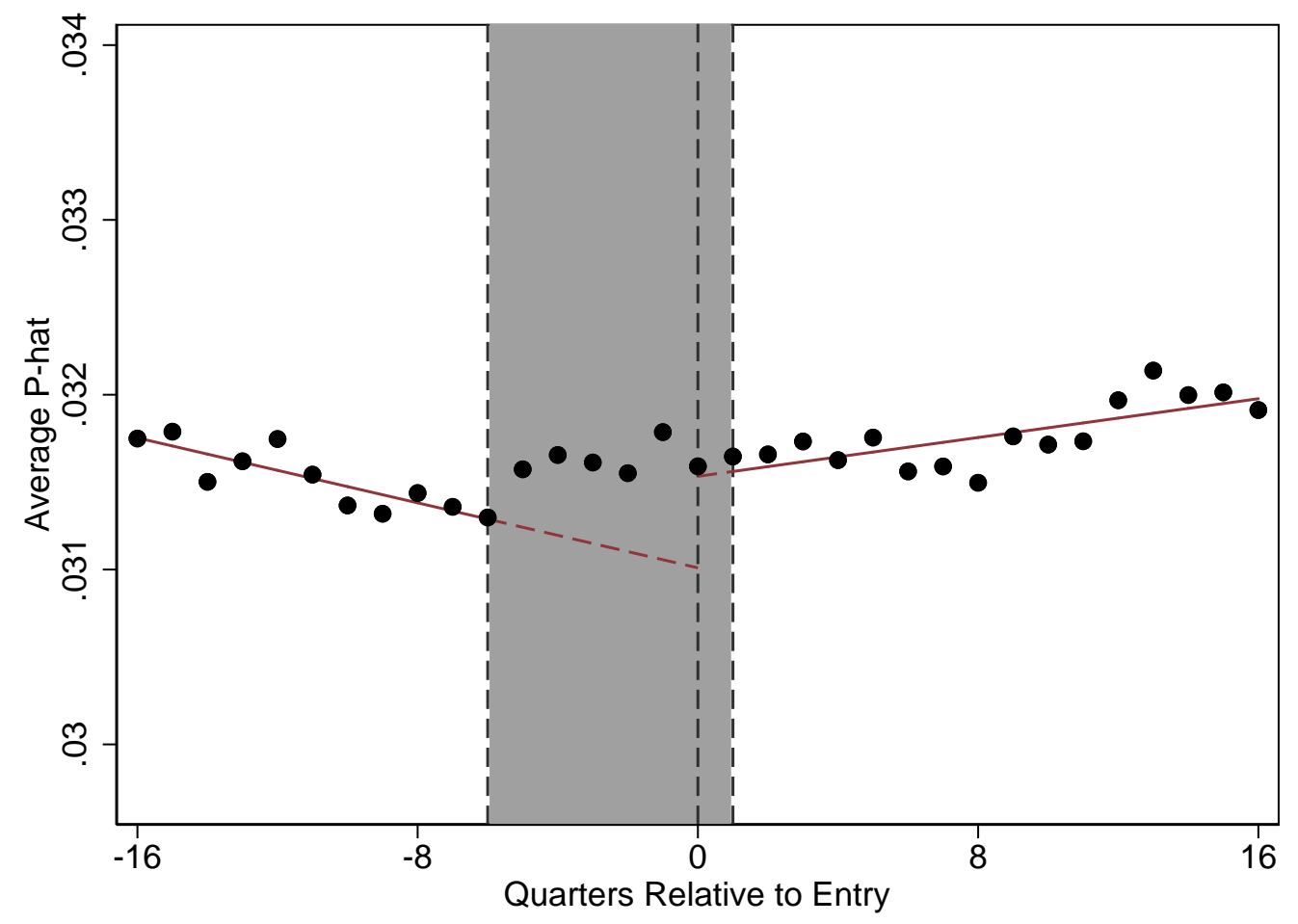

Note: Figure reports estimates of equation (1), estimated on the baseline event study sample. The figure displays our estimated function of relative quarter, $r$, and a scatter plot of the average residualized values of $\hat{p}$. Quarters $-6<r<1$ are greyed out because we drop all observations in these quarters. The y-axis reports the average $\hat{p}$ and is scaled so that the mean at $r=-1$ is equal to the mean at $r=-1$ among HSAs. 
Figure 5: Discharge to LTCH

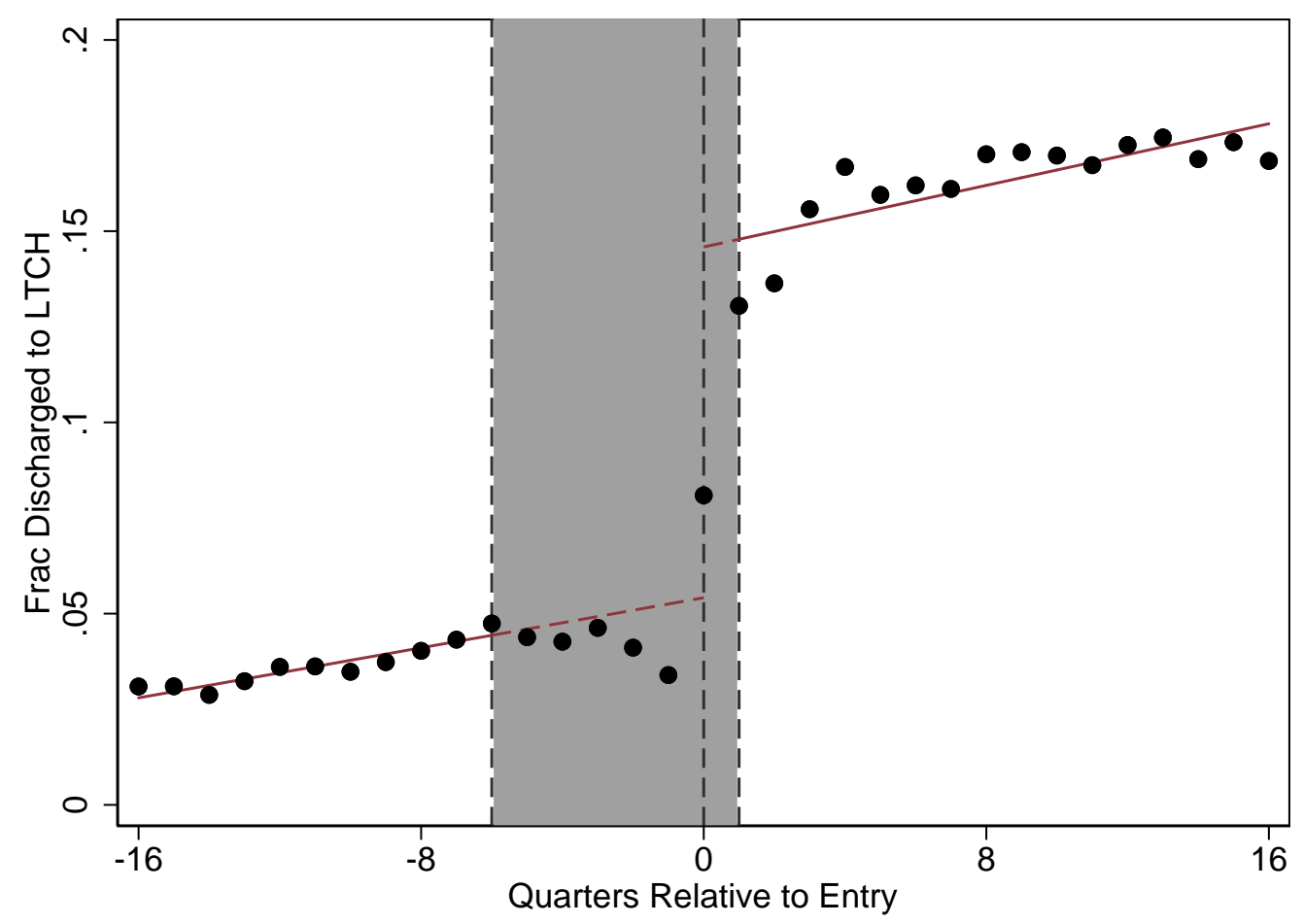

Note: Figure reports estimates of equation (1), estimated on the high $\hat{p}$ sub-sample of the baseline event study sample. The figure displays our estimated function of relative quarter, $r$, and a scatter plot of the average residualized values of the discharged to LTCH indicator. Quarters $-6<r<1$ are greyed out because we drop all observations in these quarters. The $\mathrm{y}$-axis reports the share discharged to LTCH and is scaled so that the mean at $r=-1$ is equal to the mean at $r=-1$ among HSAs. 
Figure 6: Alternative Discharge Destinations

(A) SNF

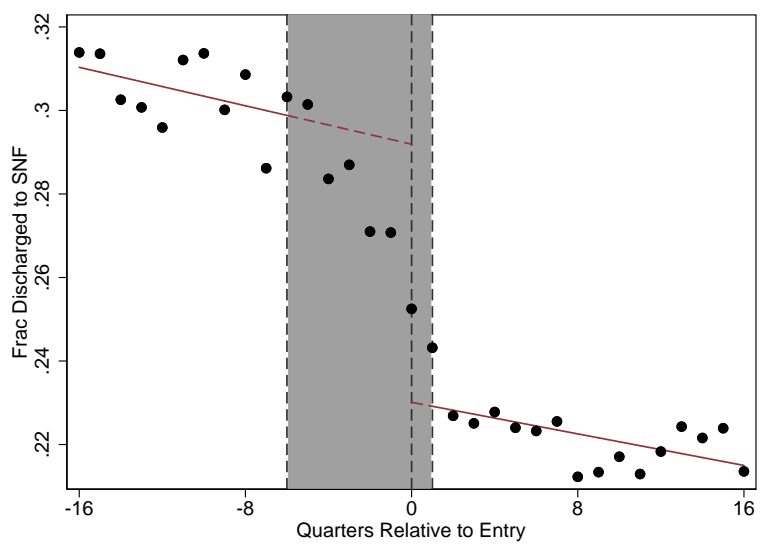

(C) LTCH-in-Training

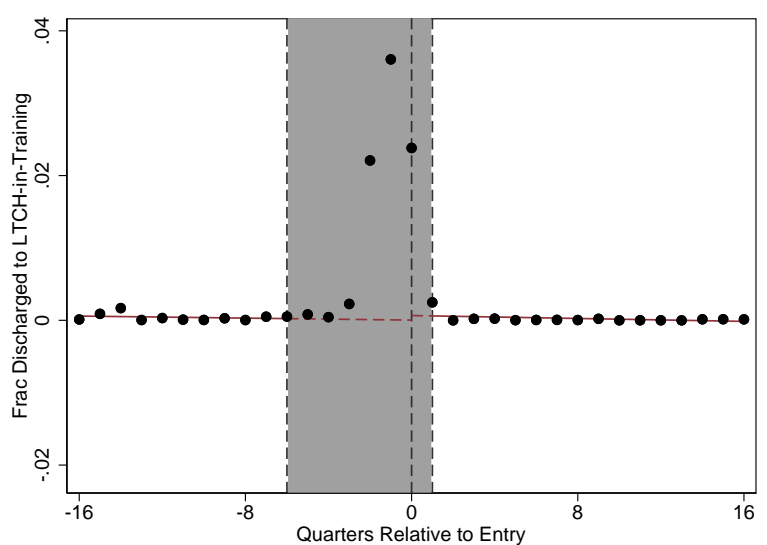

(B) Home/Other

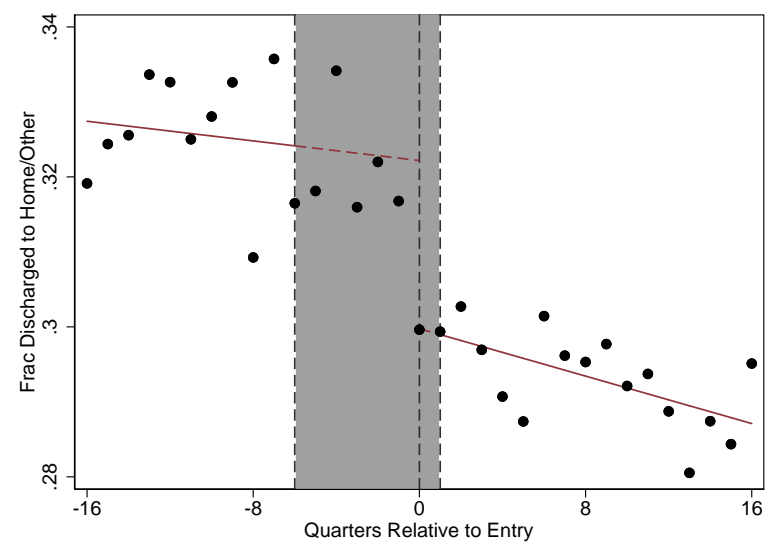

(D) $\mathrm{ACH}$

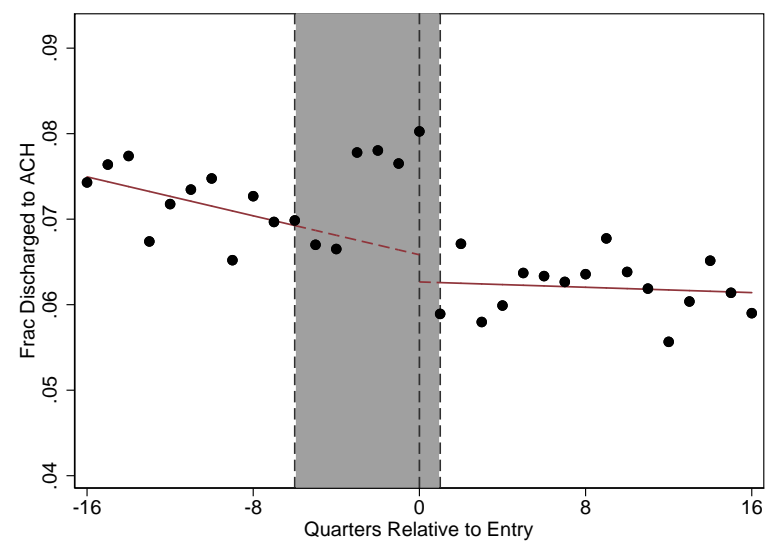

(E) Death

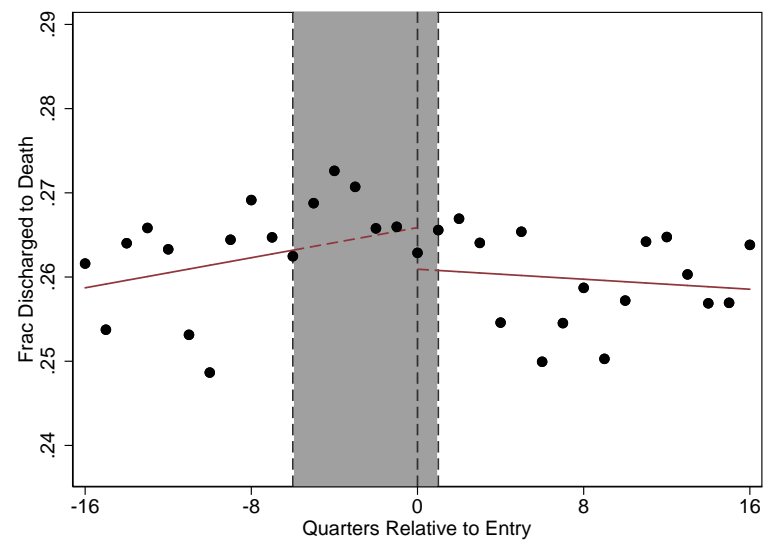

Note: Figure reports estimates of equation (1), estimated on the high $\hat{p}$ sub-sample of the baseline event study sample. The figure displays our estimated function of relative quarter, $r$, and a scatter plot of the average residualized values of each of the discharge destination indicators. Quarters $-6<r<1$ are greyed out because we drop all observations in these quarters. The y-axis reports the share discharged to the location indicated and is scaled so that the mean at $r=-1$ is equal to the outcome mean at $r=-1$ among HSAs. 
Figure 7: Total Spell Utilization

(A) Total Spell Days

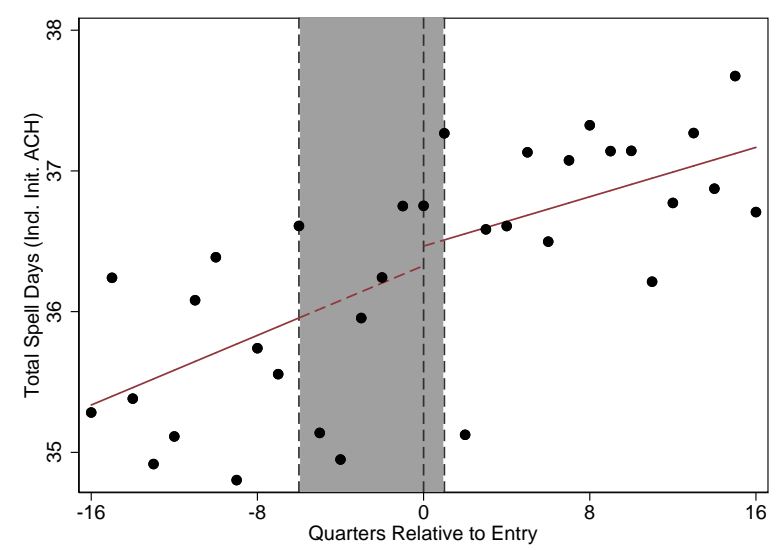

(B) Total Spell Spending

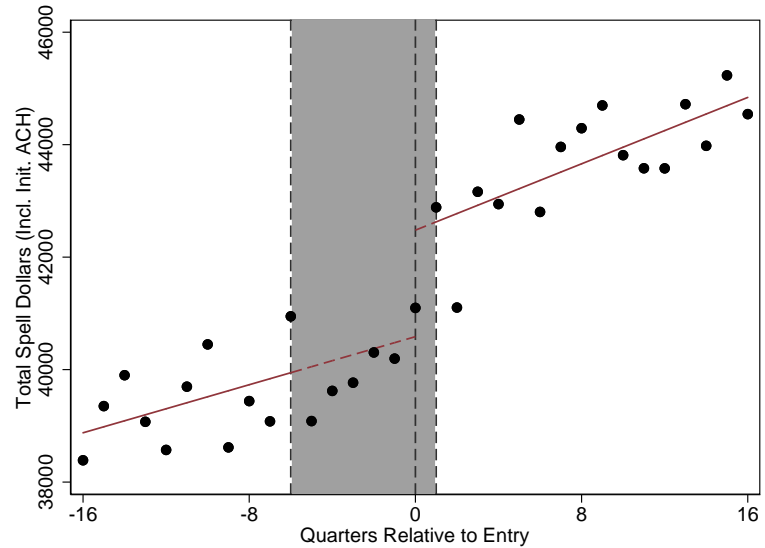

Note: Figure reports estimates of equation (1), estimated on the high $\hat{p}$ sub-sample of the baseline event study sample. The figure displays our estimated function of relative quarter, $r$, and a scatter plot of the average residualized values of spell days and spell spending. Quarters $-6<r<1$ are greyed out because we drop all observations in these quarters. The $\mathrm{y}$-axis reports the utilization measure indicated and is scaled so that the mean at $r=-1$ is equal to the outcome mean at $r=-1$ among HSAs. 
Figure 8: Patient Welfare Outcomes

(A) Total Spell Out of Pocket Spending

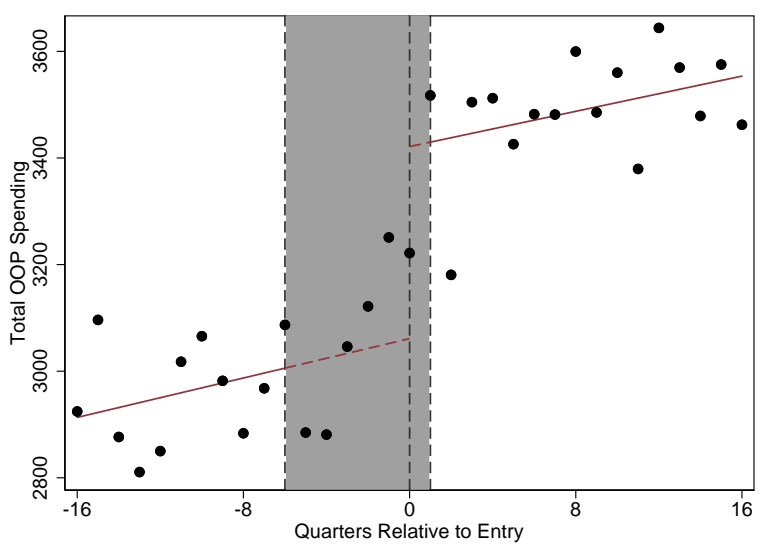

(B) Home in 90 Days

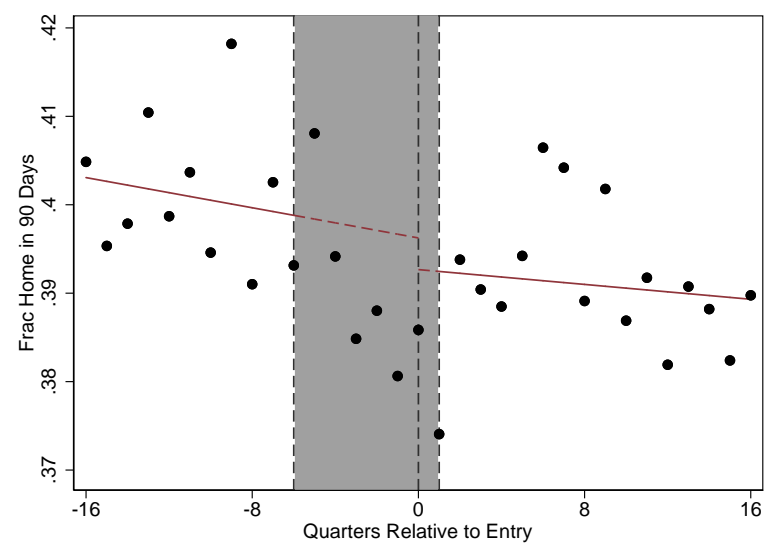

(C) 90-Day Mortality

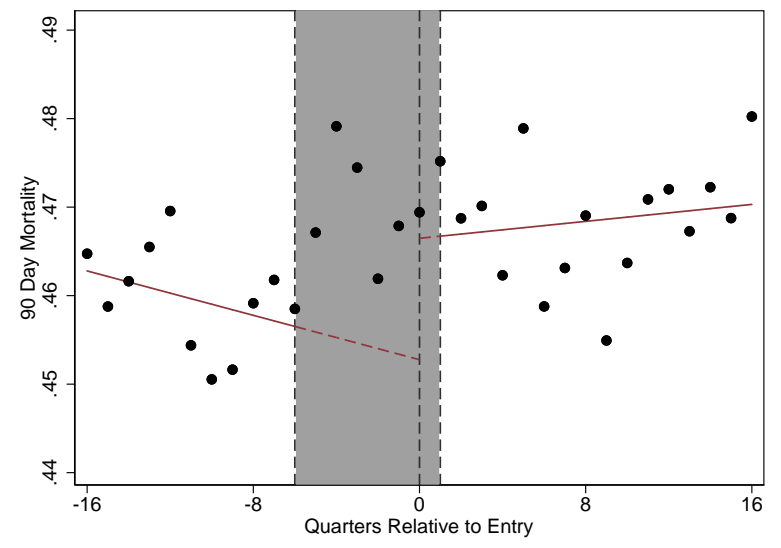

Note: Figure reports estimates of equation (1), estimated on the high $\hat{p}$ sub-sample of the baseline event study sample. The figure displays our estimated function of relative quarter, $r$, and a scatter plot of the average residualized values of each of the patient welfare outcomes. Quarters $-6<r<1$ are greyed out because we drop all observations in these quarters. The y-axis reports the utilization measure indicated and is scaled so that the mean at $r=-1$ is equal to the outcome mean at $r=-1$ among HSAs. 
Table 1: Correlates of LTCH Location

\begin{tabular}{|c|c|c|c|c|}
\hline & $\begin{array}{c}\text { HSAs w/ pre-1998 } \\
\text { LTCH } \\
\text { (1) }\end{array}$ & $\begin{array}{c}\text { HSAs w/ 1998- } \\
2014 \text { entry } \\
\text { (2) }\end{array}$ & $\begin{array}{c}\text { HSAs w/ no LTCH } \\
\text { by } 2014 \\
\text { (3) }\end{array}$ & $\begin{array}{c}\text { Correlation }^{a} \\
\text { (4) }\end{array}$ \\
\hline Number of HSAs & 152 & 186 & 3,098 & \\
\hline \multicolumn{5}{|l|}{ HSA demographics: } \\
\hline Population (000s) & 540.7 & 233.5 & 59.1 & 0.47 \\
\hline \% Urban & 0.84 & 0.76 & 0.50 & 0.28 \\
\hline$\%$ Black & 0.16 & 0.15 & 0.07 & 0.18 \\
\hline Median age & 39.6 & 40.9 & 43.2 & -0.16 \\
\hline Median income (US\$) (000s) & 56.0 & 49.9 & 49.9 & 0.05 \\
\hline$\%<\mathrm{FPL}$ & 0.16 & 0.17 & 0.16 & 0.02 \\
\hline$\%$ Uninsured & 0.14 & 0.15 & 0.14 & 0.03 \\
\hline \% Dual & 0.20 & 0.19 & 0.18 & 0.04 \\
\hline \multicolumn{5}{|l|}{ HSA hospital system: } \\
\hline ACH beds per 1,000 people & 4.2 & 4.5 & 3.6 & 0.05 \\
\hline$\%$ For profit & 0.25 & 0.25 & 0.13 & 0.11 \\
\hline$\%$ SNF discharges & 0.20 & 0.19 & 0.12 & 0.21 \\
\hline$\%$ PAC discharges & 0.41 & 0.39 & 0.21 & 0.27 \\
\hline Medicare spend per beneficiary (\$US) (000s) & 10.17 & 9.92 & 9.59 & 0.09 \\
\hline \multicolumn{5}{|l|}{ HSA region: } \\
\hline \% HSAs with LTCH in census division ${ }^{\mathrm{b}}$ & 0.12 & 0.13 & 0.10 & 0.13 \\
\hline$\%$ HSAs with LTCH in state in $1984^{c}$ & 0.50 & 0.34 & 0.39 & 0.02 \\
\hline$\%$ in states with CON law ${ }^{d}$ & 0.40 & 0.41 & 0.49 & -0.05 \\
\hline
\end{tabular}

Note: "Pre-1998 Entry" is the set of HSAs that ever had an LTCH from 1984-1997. "1998-2014 Entry" refers to HSAs where a first LTCH entered from 1998 to 2014. "Never Enter" is the set of HSAs that never had any LTCHs from 1984-2014. Population, share urban, and share black are determined from the 2010 census. Median age, income, the share below the poverty line, and the share uninsured are from the 2010-2014 ACS. ACH beds per capita, share for-profit, share discharged to SNF/IRF, share discharged to any PAC (including LTCH, SNF, IRF, HHA or hospice), and Medicare spending were calculated for 2014, the final year of observation in our event study.

${ }^{a}$ The bivariate correlation column lists the bivariate correlation between the outcome variable and an indicator for whether an HSA ever had an LTCH from 1984-2014 (i.e. the union of the HSAs in column 1 and column 2).

${ }^{b}$ Census Division's share of HSAs with an LTCH calculates the share of HSAs in the same Census division as that reference HSA that have an LTCH in 2014 (leaving out the reference HSA).

cShare with an LTCH in state in 1984 calculate the share of HSAs in each group (pre-1998 entry, 1998-2014 entry, and never entry) that had an LTCH in the state in 1984.

${ }^{d}$ Share in a state that ever had a Certificate of Need (CON) law: This is the share of HSAs in each group that is in a state that had a CON law at any point from 2002-2010. State CON laws for 2002-2010 are reported in AHPA (2003-2011). 
Table 2: Summary Statistics: Predictors of LTCH Discharge

\begin{tabular}{|c|c|c|c|}
\hline & $\begin{array}{l}\text { All } \mathrm{ACH} \text { admissions } \\
\text { (1) }\end{array}$ & $\begin{array}{c}\text { Baseline sample } \\
\text { (2) }\end{array}$ & $\begin{array}{c}\text { High } p \text {-hat sample } \\
\text { (3) }\end{array}$ \\
\hline P-hat & 0.020 & 0.033 & 0.189 \\
\hline Number of obs. (000s) & 163,649 & 90,384 & 2,338 \\
\hline \multicolumn{4}{|l|}{ Demographics } \\
\hline Age & 73.9 & 75.6 & 71.1 \\
\hline Female & 0.57 & 0.57 & 0.49 \\
\hline White & 0.83 & 0.83 & 0.77 \\
\hline Black & 0.12 & 0.12 & 0.17 \\
\hline Hispanic & 0.02 & 0.02 & 0.02 \\
\hline Asian & 0.01 & 0.01 & 0.01 \\
\hline Dual-Eligible for Medicaid & 0.26 & 0.28 & 0.34 \\
\hline \multicolumn{4}{|l|}{ Selected Features } \\
\hline Respiratory failure; insufficiency; arrest & 0.07 & 0.14 & 0.71 \\
\hline Septicemia & 0.06 & 0.11 & 0.59 \\
\hline Chronic skin ulcer & 0.04 & 0.08 & 0.31 \\
\hline Nutritional deficiencies & 0.05 & 0.09 & 0.44 \\
\hline Infective arthritis and osteomyelitis & 0.01 & 0.02 & 0.15 \\
\hline Complications of surgical procedures or medical care & 0.06 & 0.10 & 0.27 \\
\hline Shock & 0.01 & 0.02 & 0.17 \\
\hline Pneumonia & 0.11 & 0.20 & 0.44 \\
\hline Acute cerebrovascular disease & 0.03 & 0.06 & 0.10 \\
\hline Aspiration pneumonitis; food/vomitus & 0.02 & 0.04 & 0.15 \\
\hline Other aftercare & 0.06 & 0.05 & 0.01 \\
\hline Skin and subcutaneous tissue infections & 0.04 & 0.07 & 0.09 \\
\hline Bacterial Infection & 0.05 & 0.10 & 0.08 \\
\hline Intracranial injury & 0.01 & 0.01 & 0.02 \\
\hline Gangrene & 0.01 & 0.02 & 0.05 \\
\hline Paralysis & 0.02 & 0.03 & 0.07 \\
\hline Pleurisy; pneumothorax; pulmonary collapse & 0.05 & 0.06 & 0.18 \\
\hline \multicolumn{4}{|l|}{ Excluded Features } \\
\hline ICU & 0.14 & 0.17 & 0.53 \\
\hline Ventilation & 0.04 & 0.08 & 0.45 \\
\hline Over 3 days in ICU/CCU & 0.20 & 0.26 & 0.64 \\
\hline Over 8 days in ICU/CCU & 0.10 & 0.14 & 0.45 \\
\hline
\end{tabular}

Note: Each observation is a unique ACH stay. The baseline sample excludes all observations with $\hat{p} \leq 0.004$. High $\hat{p}$ stays refer to ACH stays with a predicted probability of LTCH discharge $(\hat{p})$ greater than 0.15 . The CCS (Clinical Classification Software) health predictors are those that are among the 18 most important selected features used as predictors in the LTCH discharge model, where variable importance is measured by ranking the additional $R^{2}$ each of the variables adds, summed across all the leaves of the tree. Note that 17 CCS categories are chosen; the 20th important variable is age. ICU is determined using the MedPAR ICU indicator code, and mechanical ventilation is defined using the CCS procedure code for respiratory intubation and mechanical ventilation. Age is enters the regression tree continuously. Race categories not listed include "other," "unknown," and "Native American." 
Table 3: Summary Statistics: Outcomes

\begin{tabular}{|c|c|c|c|c|c|c|}
\hline \multirow{2}{*}{ Discharge destination } & \multicolumn{2}{|c|}{$\begin{array}{l}\text { All ACH admissions } \\
\text { (1) }\end{array}$} & \multicolumn{2}{|c|}{$\begin{array}{l}\text { Baseline sample } \\
\text { (2) }\end{array}$} & \multicolumn{2}{|c|}{$\begin{array}{l}\text { High } p \text {-hat sample } \\
\text { (3) }\end{array}$} \\
\hline & & & & & & \\
\hline $\mathrm{LTCH}$ & 0.010 & $(0.101)$ & 0.018 & $(0.133)$ & 0.126 & $(0.332)$ \\
\hline SNF & 0.166 & $(0.372)$ & 0.220 & $(0.414)$ & 0.252 & $(0.434)$ \\
\hline Home/Other & 0.736 & $(0.441)$ & 0.646 & $(0.478)$ & 0.334 & $(0.472)$ \\
\hline LTCH-in-training & 0.000 & $(0.011)$ & 0.000 & $(0.014)$ & 0.001 & $(0.036)$ \\
\hline (other) $\mathrm{ACH}$ & 0.052 & $(0.221)$ & 0.056 & $(0.230)$ & 0.063 & $(0.243)$ \\
\hline Death & 0.036 & $(0.186)$ & 0.059 & $(0.236)$ & 0.224 & $(0.417)$ \\
\hline \multicolumn{7}{|l|}{ Spell days } \\
\hline $\mathrm{LTCH}$ & 0.4 & $(4.0)$ & 0.7 & $(5.1)$ & 4.3 & $(13.1)$ \\
\hline SNF & 7.0 & $(19.1)$ & 9.0 & $(21.5)$ & 12.4 & $(25.9)$ \\
\hline Initiating $\mathrm{ACH}$ & 5.4 & $(5.6)$ & 6.9 & $(6.7)$ & 16.2 & $(14.0)$ \\
\hline Total & 14.1 & $(23.3)$ & 18.0 & $(26.5)$ & 35.8 & $(38.3)$ \\
\hline \multicolumn{7}{|l|}{ Spell spending (\$) } \\
\hline $\mathrm{LTCH}$ & 530 & $(5,254)$ & 843 & $(6,657)$ & 5,869 & $(18,115)$ \\
\hline SNF & 2,692 & $(7,222)$ & 3,404 & $(8,078)$ & 4,782 & $(10,189)$ \\
\hline Initiating $\mathrm{ACH}$ & 10,079 & $(10,466)$ & 11,201 & $(12,342)$ & 27,583 & $(30,116)$ \\
\hline Total & 14,992 & $(17,665)$ & 17,519 & $(20,713)$ & 42,202 & $(44,769)$ \\
\hline \multicolumn{7}{|l|}{ Patient outcomes } \\
\hline Out-of-pocket spending (\$) & 1,507 & $(2,602)$ & 1,716 & $(3,111)$ & 3,334 & $(6,431)$ \\
\hline Home within 90 days & 0.82 & $(0.39)$ & 0.73 & $(0.44)$ & 0.42 & $(0.49)$ \\
\hline Died within 90 days & 0.14 & $(0.34)$ & 0.20 & $(0.40)$ & 0.44 & $(0.50)$ \\
\hline Mean p-hat & 0.020 & $(0.032)$ & 0.034 & $(0.039)$ & 0.189 & $(0.034)$ \\
\hline Number of Obs. (000s) & \multicolumn{2}{|c|}{24,251} & \multicolumn{2}{|c|}{13,093} & \multicolumn{2}{|c|}{373} \\
\hline
\end{tabular}

Note: Each observation is a unique ACH stay. All ACH admissions (Column 1) includes all HSAs that experience a first entry from 1998-2014, dropping observations at and after the quarter of subsequent entry or LTCH exit. Baseline sample (column 2) further excludes all observations with $\hat{p} \leq 0.004$. High $\hat{p}$ sample (Column 3 ) refers to ACH stays in the baseline sample with $\hat{p}>0.15$. 
Table 4: Event Study Estimates

\begin{tabular}{|c|c|c|c|c|c|c|}
\hline \multirow{2}{*}{ Discharge destination } & \multicolumn{2}{|c|}{$\begin{array}{l}\text { High p-hat sample } \\
\text { Reduced form } \\
\text { (1) }\end{array}$} & \multicolumn{2}{|c|}{$\begin{array}{l}\text { High p-hat sample } \\
\text { I.V. } \\
\text { (2) }\end{array}$} & \multicolumn{2}{|c|}{$\begin{array}{l}\text { Baseline sample } \\
\text { I.V. } \\
\text { (3) }\end{array}$} \\
\hline & & & & & & \\
\hline $\mathrm{LTCH}$ & 0.092 & $(0.009)$ & & & & \\
\hline SNF & -0.062 & $(0.010)$ & -0.674 & $(0.103)$ & -0.791 & $(0.075)$ \\
\hline Home/Other & -0.022 & $(0.010)$ & -0.244 & $(0.105)$ & -0.236 & $(0.073)$ \\
\hline LTCH-in-training & 0.001 & $(0.001)$ & 0.007 & $(0.006)$ & 0.007 & $(0.003)$ \\
\hline (other) ACH & -0.003 & $(0.006)$ & -0.035 & $(0.064)$ & 0.044 & $(0.040)$ \\
\hline Death & -0.005 & $(0.009)$ & -0.054 & $(0.101)$ & -0.024 & $(0.051)$ \\
\hline \multicolumn{7}{|l|}{ Spell days } \\
\hline LTCH & 2.8 & (0.4) & 30.0 & (1.9) & 28.9 & (1.0) \\
\hline SNF & -1.5 & $(0.5)$ & -16.2 & (6.3) & -14.3 & (3.9) \\
\hline Initiating $\mathrm{ACH}$ & -1.4 & $(0.4)$ & -15.5 & (4.8) & -8.6 & (2.1) \\
\hline Total & 0.1 & (0.9) & 1.5 & (9.4) & 6.6 & (5.1) \\
\hline \multicolumn{7}{|l|}{ Spell spending (\$) } \\
\hline LTCH & 3,138 & (592) & 34,210 & $(4,079)$ & 34,569 & $(1,708)$ \\
\hline SNF & -513 & (246) & $-5,593$ & $(2,714)$ & $-3,024$ & $(1,572)$ \\
\hline Initiating $\mathrm{ACH}$ & $-1,124$ & (844) & $-12,256$ & $(9,340)$ & $-2,016$ & $(3,800)$ \\
\hline Total & 1,894 & $(1,088)$ & 20,649 & $(11,065)$ & 29,583 & $(4,810)$ \\
\hline \multicolumn{7}{|l|}{ Patient outcomes } \\
\hline Out-of-pocket spending (\$) & 360 & (133) & 3,928 & $(1,381)$ & 2,420 & (640) \\
\hline Home within 90 days & -0.004 & $(0.010)$ & -0.039 & $(0.110)$ & -0.172 & $(0.088)$ \\
\hline Died within 90 days & 0.014 & $(0.010)$ & 0.150 & $(0.109)$ & 0.101 & $(0.065)$ \\
\hline Number of Obs. (000s) & \multicolumn{2}{|c|}{343} & \multicolumn{2}{|c|}{343} & \multicolumn{2}{|c|}{11,824} \\
\hline
\end{tabular}

Note: Column 1 reports estimates and standard errors of $\alpha$ in equation (1) and column 2 reports the IV estimate and standard errors of $\beta^{y}$ from equations (2) and (3), both estimated on the high $\hat{p}$ sub-sample of the baseline event study sample. Column 3 reports IV estimates and standard errors of $\beta^{y}$ from equations (4) and (5), estimated on the baseline event study sample. Standard errors are clustered at the HSA level (186 clusters) for the high $\hat{p}$ sample (columns 1 and 2), and at the HSA-bin level (930 clusters) for the baseline specification (column 3). 
Table 5: Event Study Estimates: Heterogeneity

\begin{tabular}{|c|c|c|c|c|c|c|c|c|}
\hline \multirow{2}{*}{ Panel A: Days in ICU / CCL } & \multirow{2}{*}{\multicolumn{2}{|c|}{$\begin{array}{l}\text { High p-hat sample } \\
\text { First stage } \\
\text { Discharge to LTCH } \\
\text { (1) }\end{array}$}} & \multicolumn{6}{|c|}{$\begin{array}{c}\text { Baseline sample } \\
\text { I.V. }\end{array}$} \\
\hline & & & \multicolumn{2}{|c|}{$\begin{array}{l}\text { Discharge to SNF } \\
\text { (2) }\end{array}$} & \multicolumn{2}{|c|}{$\begin{array}{c}\text { Total spell spending (\$) } \\
\text { (3) }\end{array}$} & \multicolumn{2}{|c|}{$\begin{array}{c}\text { Died within } 90 \text { days } \\
\text { (4) }\end{array}$} \\
\hline Over 3 days & 0.105 & $(0.011)$ & -0.689 & $(0.069)$ & 31,935 & $(6,472)$ & 0.176 & $(0.070)$ \\
\hline Under 3 days & 0.067 & $(0.008)$ & -1.015 & $(0.137)$ & 25,818 & $(5,345)$ & -0.015 & $(0.120)$ \\
\hline \multicolumn{9}{|c|}{ Panel B: Mechanical ventilator } \\
\hline On a ventilator & 0.094 & $(0.012)$ & -0.673 & $(0.074)$ & 40,788 & $(2,593)$ & 0.221 & (0.097) \\
\hline Not on a ventilator & 0.091 & $(0.009)$ & -0.851 & $(0.098)$ & 31,624 & $(1,531)$ & -0.003 & $(0.077)$ \\
\hline \multicolumn{9}{|c|}{ Panel C: Pre-entry LTCH discharge rate } \\
\hline Above median & 0.072 & $(0.015)$ & -0.765 & $(0.121)$ & 30,983 & $(3,167)$ & 0.276 & (0.111) \\
\hline Below median & 0.113 & $(0.011)$ & -0.713 & $(0.085)$ & 36,363 & $(1,582)$ & 0.014 & $(0.077)$ \\
\hline \multicolumn{9}{|l|}{ Panel D: For-profit status } \\
\hline For-profit LTCH & 0.091 & $(0.011)$ & -0.745 & $(0.101)$ & 34,447 & $(2,118)$ & 0.192 & $(0.080)$ \\
\hline Not-for-profit LTCH & 0.095 & $(0.015)$ & -0.824 & $(0.113)$ & 35,546 & $(3,383)$ & -0.062 & (0.109) \\
\hline
\end{tabular}

Note: Column 1 reports first stage estimates and standard errors of $\alpha$ in equation (1) in the high $\hat{p}$ sample. Columns 2-4 reports the IV estimate and standard errors of $\beta^{y}$ from equations (4) and (5), estimated on the baseline event study sample. Standard errors are clustered at the HSA level (186 clusters) for the high $\hat{p}$ sample (columns 1), and at the HSA-bin level (930 clusters) for the baseline specification (column 3). In Panel C, the pre-entry discharge rate is based on the rate in period $r=-6$. For this panel, we exclude 30 of the 186 MSAs where we do not observe outcomes in period $r=-6$. 


\title{
Long-Term Care Hospitals: A Case Study in Waste Online Appendix
}

\author{
Liran Einav Amy Finkelstein Neale Mahoney
}

\section{A Data Appendix}

\section{A.1 Defining LTCH Entry}

We define LTCH entry using both the Medicare claims in the MedPAR dataset and the Provider of Service (POS) File. We determine the precise timing of entry with the MedPAR data, defining the quarter of entry as the first quarter in which we observe a stay at a given LTCH facility. Appendix Figure A4 confirms that the first stay is a good indicator of facility entrance. There is a large jump in admissions to the entering LTCH in the week of the first stay as the LTCH fills its beds, followed by a more gradual scale up from three to four admissions per week over the course of the LTCH's first six months.

The Medicare claims data do not include facility geography. We rely on the annual Provider of Service (POS) File, which records characteristics of Medicare-approved facilities, to match each LTCH to a given geography (an HSA in our baseline specification). Because we are using two different data sources, Appendix Figure A5 cross-validates the timing of LTCH entry between our two sources. In both data sets, we define entry as the first time the facility "appears" in the data (the first stay in the MedPAR data and the first entry in the POS file). The size of the dots in Appendix Figure A5 correspond to the number of LTCHs that are defined as entering in a given (year MedPAR, $_{\text {ear }}$ POS) pair. The majority of entrances lie on the 45-degree line, indicating that our two data sets generally agree. Nearly all entries that do not align have a lagged date of entry in the POS file, potentially indicating some administrative lag between when an LTCH is established and when it makes its way into the POS File. In these cases, we backfill the geography of the facility from the first instance it appears in the POS data to the quarter we identify the first stay associated with a given LTCH facility.

\section{A.2 Variable Definitions}

Discharge Destinations We define a patient as being discharged to a given facility if they are admitted to that facility within one day of discharge from the initiating ACH stay. Although each stay-level record in the MedPAR data contains an indicator for the discharge destination of each patient (e.g., $\mathrm{ACH}, \mathrm{SNF}$, home, death), we construct our own definition of discharge destination within a healthcare spell. We do so primarily because the "discharge to LTCH" code was first introduced in 2002; prior to 2002, discharges to LTCHs were grouped into an "other" category. In addition, the destination codes are often unreliable; in many instances following $\mathrm{ACH}$ discharge, a patient is immediately admitted at a facility type inconsistent with the recorded discharge destination (Kahn and Iwashyna, 2010). We code the discharge destination for a patient that died at the $\mathrm{ACH}$ as "discharged to death." We code the discharge destination for a patient that is still alive following $\mathrm{ACH}$ discharge but not making any Medicare payments to an ACH, LTCH, SNF, or IRF as discharged to home without care or to some other care, such as a hospice or home health care. 
Spell Definition, Days, and Spending As in Einav, Finkelstein and Mahoney (2018), we define spells as the set of almost-continuous days with a Medicare payment to an acute care hospital, LTCH, SNF, or IRF following an acute care hospital stay. The spell starts on the first day of the ACH stay and ends if there are two consecutive days without any Medicare payments to any of these institutions. Spell days count the number of days in each facility over the course of the spell, including the initiating acute care stay. Spell spending sums Medicare reimbursements, excluding outlier payments, at each facility over the spell, including the initiating acute care stay. Out-of-pocket spending sums the beneficiary coinsurance, inpatient deductible, and blood deductible at each facility over the spell, including the initiating $\mathrm{ACH}$ stay.

Patient Outcomes We have three main patient outcome measures. The first is whether the patient is home within 90 days of admission to the initiating acute care hospital stay. If the discharge destination code in the final facility within a spell of continuous days is to home (with either self-care or in the care of a home health association) within 90 days of the date of admission at the initiating acute care stay, the patient is coded as going home within 90 days. The second is an indicator for whether the beneficiary dies within 90 days of the date of admission of the index ACH stay. The third is the amount of out-of-pocket spending owed for the spell - i.e., payments not covered by Medicare; these payments may be covered by the patient's supplemental insurance plan.

LTCHs-in-Training Before a facility can be classified as an LTCH, it must demonstrate a 25-day average length of stay for six months ( 42 CFR $\S 412.23,2011)$. Because of this regulatory requirement, an LTCH must begin its life as another facility (which we call an "LTCH-in-training"), most commonly acute care hospitals. When an LTCH-in-training is reclassified as an LTCH, it remains the same facility in all operational senses (the same building, the same services, etc.), but its reimbursement schedule changes. As we will show, the vast majority of LTCHs-in-training exist for only the six months required to fulfill the regulatory requirement. Because these facilities are neither true ACHs (since they are operationally an LTCH) nor true LTCHs (since they are not reimbursed as such), we classify them as a separate entity.

The facility's provider identification number changes upon reclassification, leaving no clear link between an LTCH and its associated LTCH-in-training in the data. To address this challenge, we rely on addresses and facility names in the POS data. The quality of the POS addresses is variable; addresses and facility names will often change slightly year over year. Moreover, it is common for multiple facilities to be located at the same address; about half of LTCHs are co-located with an ACH in "Hospitals within Hospitals," rendering a simple match on addresses insufficient. To address these data challenges, we searched for LTCHs-in-training by hand using addresses and facility names in the POS data.

We first analyze the address and facility name data by hand, defining a potential LTCH-in-training as any facility that meets the following two criteria: (1) exists at the same address as an entering LTCH in the year of entry or prior to entry, and (2) vanishes from the data in the year following entry. Using this method, we were able to identify 192 total potential LTCHs-in-training. Encouragingly, these 192 potential LTCHs-in-training have an average length of stay of just over 25 days in the six months prior 
to entry, as required by CMS regulation. Among the potential LTCHs-in-training we identified by hand, we found that the 158 (82\%) are ACHs (the remainder are IRFs). For simplicity, we only consider ACHs that precede LTCHs as LTCHs-in-training. Of these 158 LTCHs-in-training, all facilities had at least one quarter with an average length of stay longer than 20 days, and 90\% had at least one quarter with an average length of stay longer than 25 days. We are able to identify the LTCHs-in-training associated with 112 of the 186 first entries we study in our main specification. Appendix Figure A6 presents a histogram of the number of quarters a facility exists for the 158 LTCHs-in-training we were able to identify. Over $90 \%$ of the LTCHs-in-training we identified exist for one year or less, implying that the vast majority of LTCHs-in-training are simply a regulatory object that allow a true LTCH to enter a market. Some LTCHs-in-training have longer facility lives, which indicates that a few facilities may have reclassified as LTCHs as their average length of stay increased organically over time.

Our method of identifying LTCHs-in-training is imperfect; we fail to identify the LTCH-in-training associated with 74 of the LTCH entries we study in our baseline analysis. Because the majority of LTCHs-in-training existed as ACHs, this means that there may be up to 74 facilities in our data that we are mischaracterizing as ACHs, when they would be more accurately described as LTCHs-in-training. In our event study analysis, Figure 6 Panel (D) shows some evidence of an increase in discharges to ACHs during the transition period; this may reflect discharges to LTCHs-in-training that we did not classify using our algorithm. To assess this, Appendix Figure A7 presents discharges to ACH restricted to the $112 \mathrm{HSAs}$ in which we were able to identify a credible LTCH-in-training associated with the entering LTCH. This restriction eliminates the increase in discharges to an ACH during the transition period, indicating that the slight increase in Figure 6 Panel (D) may be due to unclassified LTCHs-in-training.

Patient Classifications In Appendix Table 5 we analyze the impact of LTCHs across patients based on the number of days they spent in an ICU or CCU during the initial ACH stay, and whether or not they were ventilated during the initial ACH stay. We identify ventilator use in the MedPAR data using the ICD-9 codes associated with the initial ACH stays. A patient is coded as "ventilated" if the stay has an ICD-9 code included in the "Respiratory intubation and mechanical ventilation" category of the Clinical Classification Software (CCS) procedure codes. Note that there is no way in the data to tell how long a patient was on a mechanical ventilator, so the "ventilated" classification is a rough approximation of the category that will be included in LTCH reimbursement under the new rules (ventilated for over 96 hours), and will include many patients who in fact do not qualify for the LTCH rate.

The number of days in an ICU (intensive care unit) and CCU (coronary care unit) for each stay are reported in the MedPAR data. A spell is included in the "Over 3 (8) days in an ICU/CCU" if the patient spent over 3 (8) days in an ICU or over 3 (8) days in a CCU during the initial ACH stay.

\section{B Constructing LTCH Discharge Probabilities ( $\hat{p} \mathbf{s})$}

We use a regression tree to generate predicted probabilities of LTCH discharge $(\hat{p})$ conditional on having an LTCH nearby. Each observation in the training and prediction data is an ACH stay from 1998-2014 with an indicator for whether a patient was discharged to an LTCH as the dependent vari- 
able. We include as predictors the calendar year of the patient's admission, the patient's age, sex, race, and an indicator for dual enrollment in Medicaid. We also use the ICD-9 diagnoses from the patient's initiating hospital admission as health status indicators. Each $\mathrm{ACH}$ stay can have up to 9 distinct diagnoses, and we cluster each diagnosis into 285 mutually exclusive Clinical Classification Software (CCS) codes (HCUP, 2017).

We construct a training set consisting of ACH stays that are "close" to an LTCH. We define close as all ACHs with an LTCH less than five kilometers away, where distance is measured using the latitudelongitude coordinates for each facility reported in the AHA Annual Survey. From 1998-2014, there were 32,724,774 "close" ACH stays. Our final training set for the model uses a 10\% random sample of these close stays, resulting in a training set of 3,272,477 ACH stays. We use 5-fold cross validation to choose a complexity parameter to maximize out of sample $R^{2}$. The complexity parameter determines how much to "prune" the regression tree; specifically, the complexity parameter is the minimum $R^{2}$ each additional leaf of the tree must add to be included in the decision tree. Appendix Figure A8 plots the cross-validated (out of sample) $R^{2}$ for different values of the complexity parameter. As the magnitude of the complexity parameters decreases, the size of the tree increases (as more new leaves cross the minimum $R^{2}$ threshold). Appendix Figure A8 shows a maximum $R^{2}$ is achieved at $3 \times 10^{-5}$. We build the optimally pruned tree (complexity parameter $=3 \times 10^{-5}$ ). As anticipated with a small complexity parameter, this creates a very large tree. For ease of visualization, we present a list of selected features in Appendix Table A6 instead of the very complex decision tree.

Appendix Table A7 presents the distribution of $\hat{p}$ (predicted from our optimally pruned tree) among all ACH stays and among ACH stays with a high $\hat{p}(\hat{p}>0.15)$, and among all "close" $\mathrm{ACH}$ stays, the set of ACH stays with an LTCH within five kilometers. Note that the mean $\hat{p}$ among all stays is higher than the share discharged to LTCHs. This is because $\hat{p}$ is the probability of LTCH discharge conditional on having an LTCH nearby; among all close ACH stays, the mean $\hat{p}$ and the share discharged to an LTCH are equivalent.

As discussed in the main text, we carefully chose predictors that are plausibly exogenous to the LTCH discharge decision. We excluded any procedures from the set of predictors because certain procedures may become more or less common when there is an LTCH available in the HSA. Panels A and B of Appendix Figure A9 present the probability of receiving a tracheostomy (a procedure to insert a breathing tube into a windpipe for a patient on a mechanical ventilator) and the probability of being on a mechanical ventilator upon LTCH entry estimated among all high $\hat{p}$ stays. Neither tracheostomy nor mechanical ventilation appear to increase upon LTCH entry, but we cannot rule out non-negligible effects.

\section{Additional Results and Robustness}

Alternative transition periods: Our baseline specification drops the $r \in[-5,0]$ transition period. Appendix Figure A10 shows first stage plots of the effect of LTCH entry on LTCH stays for alternative transition periods. Specifically, we show results for a narrow transition period which drops quarters $r \in[-2,0]$ and a wide transition period which drops quarters $r=[-5,5]$. Appendix Table A8 reports our baseline IV estimates for these narrow and wide periods. Both the plots and tables confirm that our results are robust to alternative transition periods. 
Alternative specifications: In Appendix Figure A11 and Appendix Table A9, we show first stage plots and IV estimates that probe the sensitivity of our results to a number of different alternative specifications. Panel A and column 1 show our baseline specification for reference. In Panel B and column 2, we show our results are robust to including all HSAs that already had at least one LTCH as of 1998 as controls. In Panel C and column 3, we restrict the sample to a balanced panel of HSAs where we can observe outcomes +/- 3 years around entry. Restricting to a balanced panel allows us to assess whether changes in sample composition (in combination with heterogeneous treatment effects) generate time-trends in our results. A comparison of the balanced panel plot with the baseline specification plot indicates little reason to be concerned about this issue.

In Panel D and column 4, we show our results are robust to including subsequent entries, rather than just the first entry into an HSA, in our event study sample. We construct the data so that HSAs with two entries "appear" twice, with the event-time variable $r$ defined in relation to the entry of interest. We additionally control for the non-focal entry in this specification. In Panel E and column 5, we show that our results are robust to dropping individuals who are dually eligible for Medicaid. Since dual eligibles have their out-of-pocket costs covered by Medicaid, it is interesting that there is a similar increase in the out of pocket costs when these patients are excluded.

The remaining panels and columns show that our results are robust to defining health care markets at alternative geographic levels. In Panel F and column 5, we show results where we redefine our event study to analyze the first entry of an LTCH into a ZIP code, and replace the HSA fixed effects with fixed effects at the ZIP code level. There are 230 first entries at the ZIP code level in comparison to 196 first entries in our baseline HSA level analysis. In Panel G and column 6, we show analogous results where were redefine our event study to analyze the first entry at the county level. There are 148 first LTCH entries at the county level in our data.

Heterogeneous first stage and homogenous second stage: Our baseline specification allowed the first stage of LTCH entry on LTCH discharge to be heterogeneous based on the five $\hat{p}$ groups, but imposed a homogeneous second stage of LTCH discharge on outcomes. Appendix Table A2 shows IV estimates separately for each of the five $\hat{p}$ groups. The IV estimates are similar across the $\hat{p}$ groups, consistent with our homogeneity assumption. Appendix Table A10 shows IV estimates where we impose a homogenous first stage (and maintain the homogenous second stage from our baseline specification). As expected, imposing a homogeneous first stage results in substantially less precise IV estimates.

Alternative spell definitions: In our baseline sample, we defined as spell as starting on the first day of the ACH stay and ending if there are two consecutive days without Medicare payments to any institution. As a robustness check, we explore an alternative spell definition, where we define a spell as the 365 days post admission to the ACH stay. Appendix Table A11 shows summary statistics with our baseline and this alternative spell definition. With the 365-day spell definition, average LTCH and SNF days and spending are roughly twice as large as under our baseline definition. Appendix Table A12 shows IV estimates on spell days and spending in both samples. The estimates with the 365-day spell sample are twice as large as those in the baseline sample, which is consistent with the 
twice-as-large averages for this sample.

Decomposing discharges to "home/other": A limitation to our baseline data is that we cannot see any finer granularity on the discharge destination of "home/other." However, for a subset of our study period (2002-2014), we have separate data on claims for home health and hospice. Using these data, we define two new destinations within the discharge to "home/other" category based upon our methodology defined in Appendix A: a patient is defined as discharged to home health or hospice if the patient has a claim for home health or hospice beginning within one day of discharge from the initiating $\mathrm{ACH}$ stay. To further decompose the remaining unclassified patients, we also use the discharge destination codes on each $\mathrm{ACH}$ claim to categorize patients as discharged "home with no care." All remaining unclassified patients are categorized as discharged to "other." Column 3 of Appendix Table A12 shows the results. When restricting to stays from 2002-2014, each LTCH discharge reduces discharges to home/other by 32.7 percentage points. We can decompose this into a decrease in discharges home with no care (which decreases by 11.4 percentage points), home with home health care (which increases by 1.5 percentage points), hospice (decreases by 7.0 percentage points) and other (which decreases by 15.8 percentage points).

Including payments to home health and hospice: A potential limitation of our baseline analysis is that the MedPAR data do not include payments to home health or hospice. We have separate data on such payments from 2002-2014. Appendix Table A11 shows these destinations account for a relatively low share of spell spending (about 10\% combined). Column 3 of Appendix Table A12 shows that incorporating home health spending into the analysis does not meaningfully impact our findings.

Including outlier payments: As discussed in Appendix Section A, our baseline measure of Medicare spending does not include outlier payments. In Appendix Table A13 and Appendix Figure A12 we show, in addition to the baseline effects for reference, effects on outlier payments and combined baseline and outlier payments. The point estimates indicate that including outlier payments strengthen our finding that LTCHs leads to excess spending. However, because the effects on outlier spending are very noisy (outlier payments are naturally rare) including them substantially decreases the precision of our results. 


\section{Figure A1: Spell Utilization by Destination}

(A) LTCH Spell Days

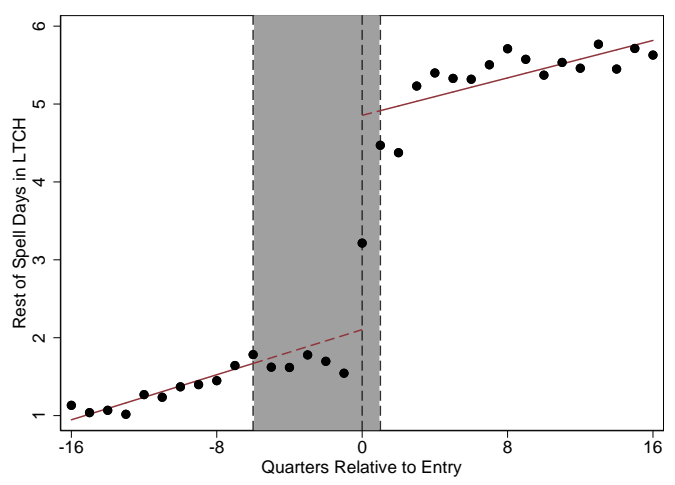

(C) SNF Spell Days

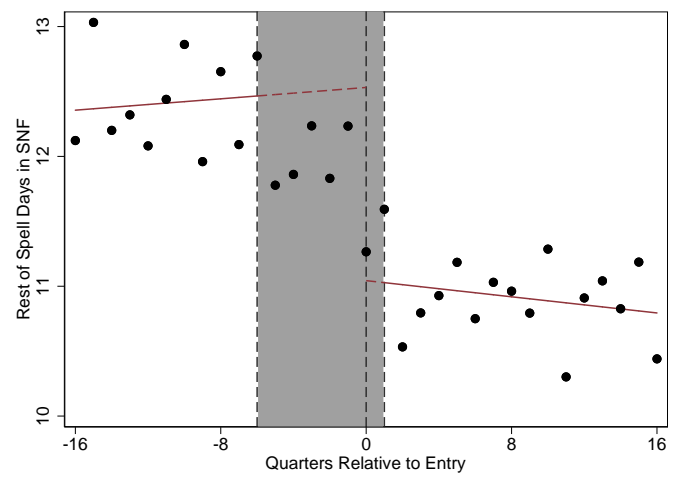

(E) Initiating ACH Days

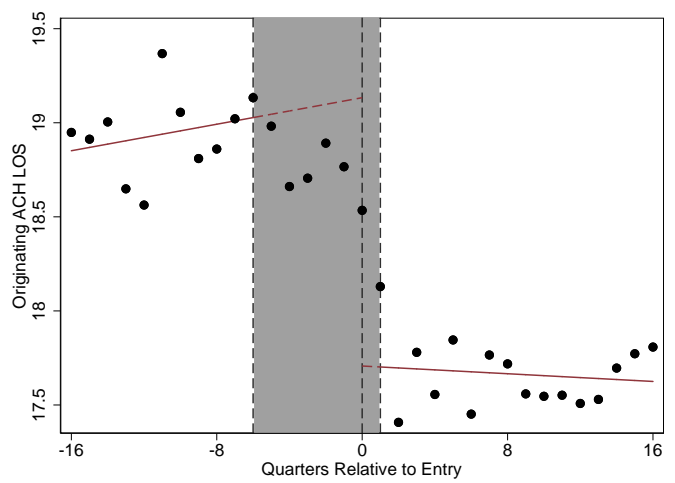

(B) LTCH Spell Spending

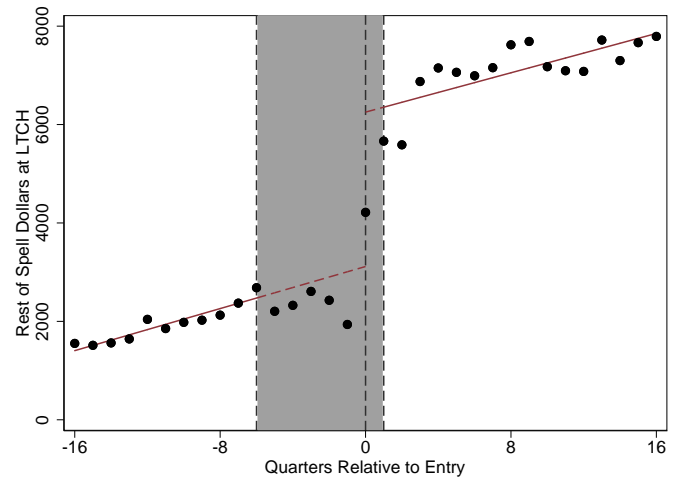

(D) SNF Spell Spending

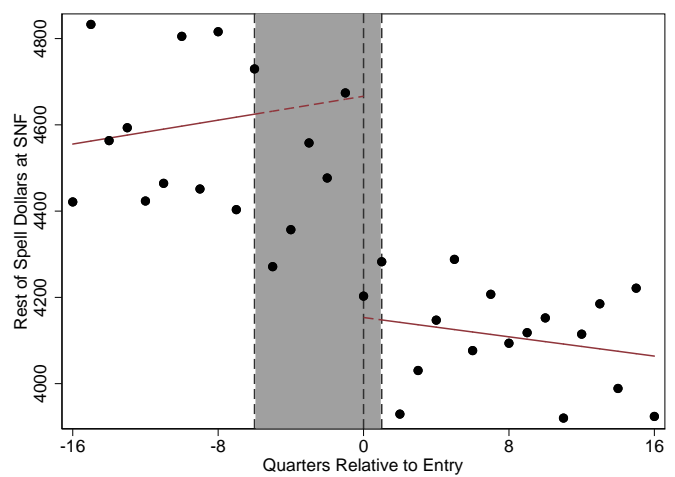

(F) Initiating ACH Spending

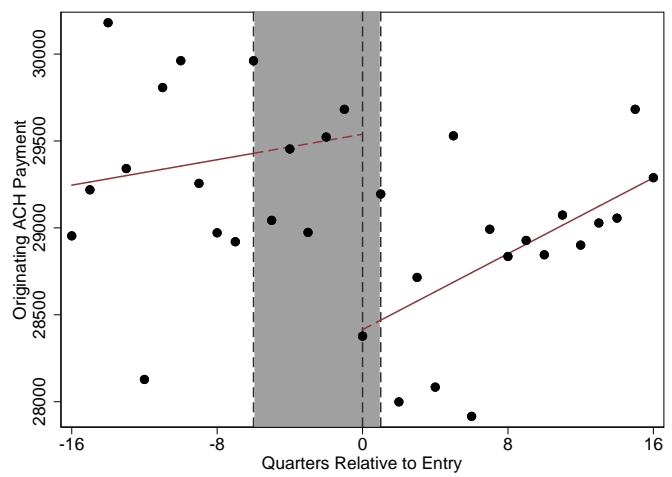

Note: Figure reports estimates of equation (1), estimated on the high $\hat{p}$ sub-sample of the baseline event study sample. The figure displays our estimated function of relative quarter, $r$, and a scatter plot of the average residualized values of the spell utilization by destination. Quarters $-6<r<1$ are greyed out because we drop all observations in these quarters. The $\mathrm{y}$-axis reports the spell utilization indicated and is scaled so that the mean at $r=-1$ is equal to the outcome mean at $r=-1$ among HSAs. 
Figure A2: First Stage: Heterogeneity by ICU /CCU and Mechanical Ventilator

(A) 3 or More Days in ICU/CCU

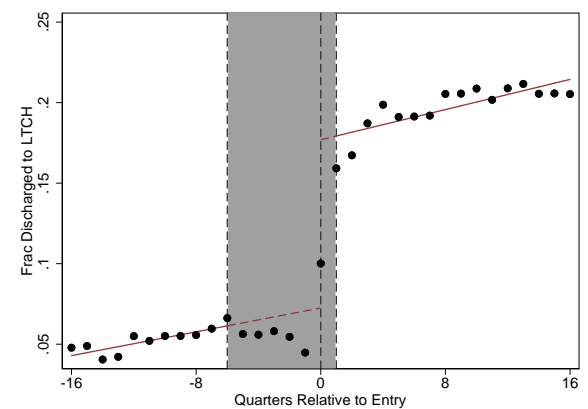

(C) 8 or More Days in ICU/CCU

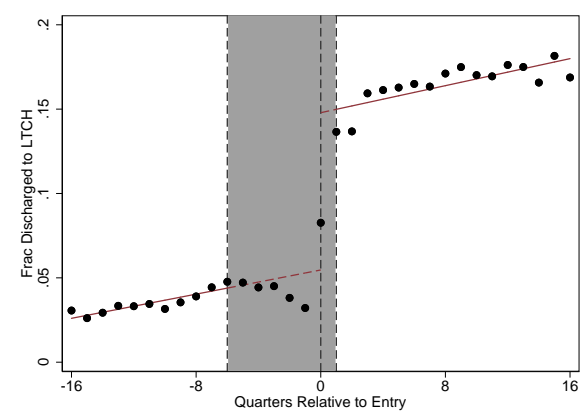

(E) Patient on Ventilator

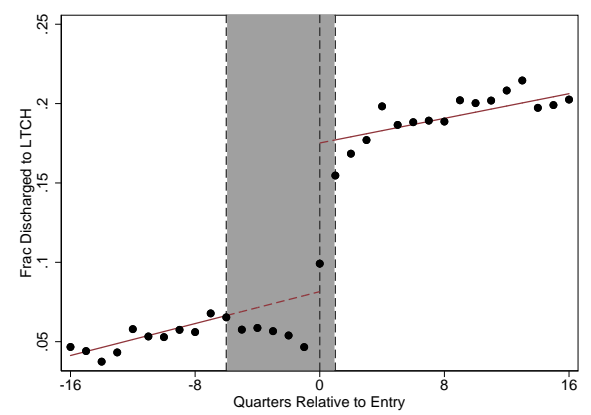

(B) Under 3 Days in ICU/CCU

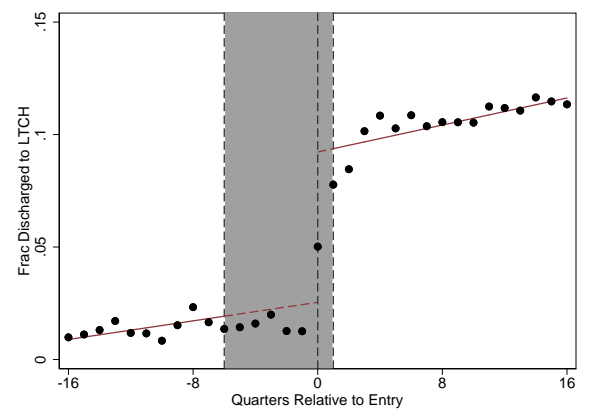

(D) Under 8 Days in ICU/CCU

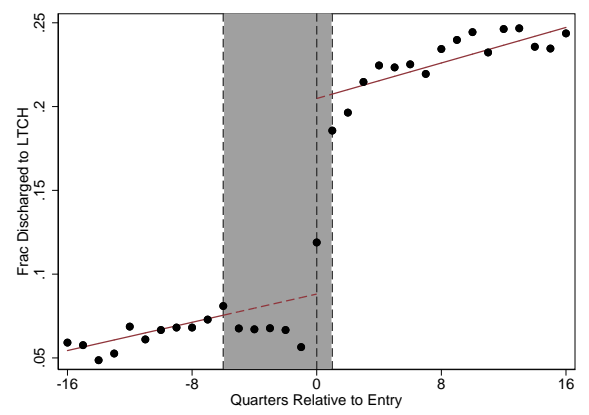

(F) Patient not on Ventilator

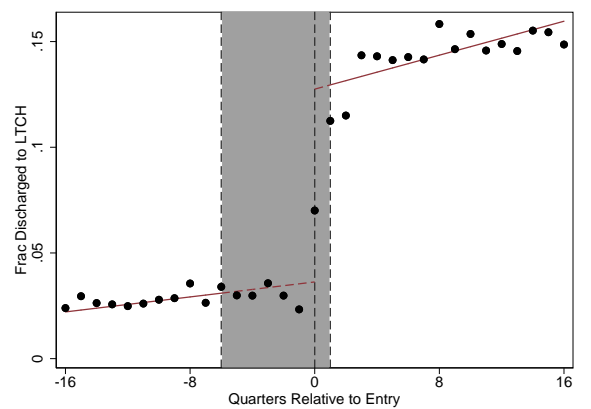

Note: Figure reports estimates of equation (1), estimated on the high $\hat{p}$ sub-sample of various robustness event study samples. Figure displays our estimated function of relative quarter, $r$, and a scatter plot of the average residualized values of the discharge to LTCH indicator. Quarters $-6<r<1$ are greyed out because we drop all observations in these quarters. Panels A and B show plots separately by whether the patient spent 3 or more days in an intensive care unit (ICU) or coronary care unit (CCU) prior to LTCH discharge. Panels C and D show plots separately by whether the patient spent 8 or more days in a ICU / CCU. Panels E and F split the analysis by whether was placed on a mechanical ventilator at the initiating ACH. The y-axis reports the share discharged to LTCH and is scaled so that the mean at $r=-1$ is equal to the outcome mean at $r=-1$ among the geographies in the sample. 


\section{Figure A3: First Stage: Heterogeneity by Pre-Entry Discharge and For-Profit Status}

(A) Below Median Pre-Entry Discharge Rate (B) Above Median Pre-Entry Discharge Rate
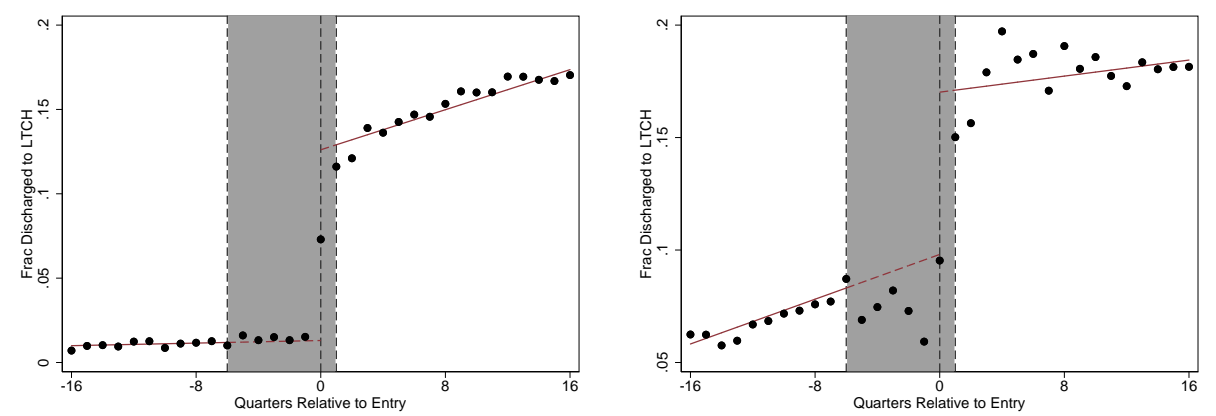

(C) Bottom Quartile Pre-Entry Discharge Rate

(D) Top Quartile Pre-Entry Discharge Rate
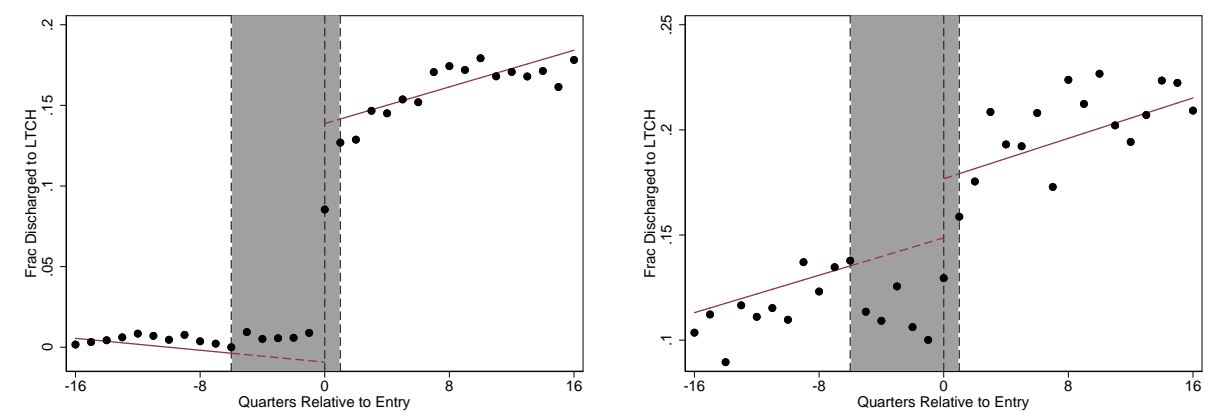

(E) For Profit

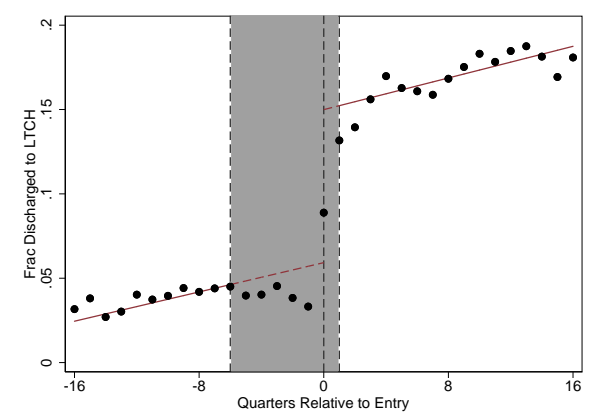

(F) Not For Profit

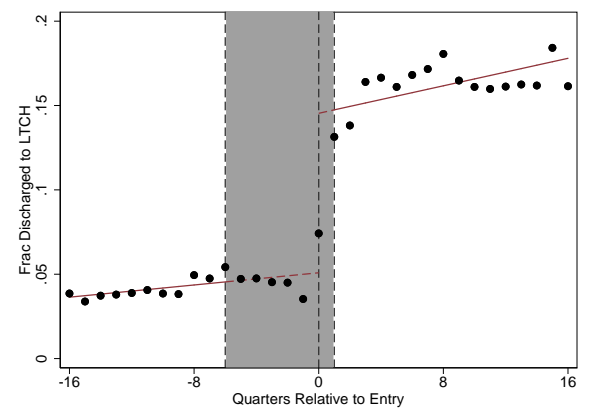

Note: Figure reports estimates of equation (1), estimated on the high $\hat{p}$ sub-sample of various robustness event study samples. Figure displays our estimated function of relative quarter, $r$, and a scatter plot of the average residualized values of the discharge to LTCH indicator. Quarters $-6<r<1$ are greyed out because we drop all observations in these quarters. Panels A and B split the sample by whether the MSA had below or above the median LTCH discharge share. Panels C and D show results for the bottom and top quartiles of LTCH discharge share. Panels E and F split the analysis by whether the LTCH is a for-profit or non-for-profit organization. In Panels A-D, the pre-entry discharge rate is based on the rate in period $r=-6$. For these panels, we exclude 30 of the 186 MSAs where we do not observe outcomes in period $r=-6$. The $\mathrm{y}$-axis reports the share discharged to LTCH and is scaled so that the mean at $r=-1$ is equal to the outcome mean at $r=-1$ among the geographies in the sample. 
Figure A4: Average Weekly Admissions Following LTCH Entry

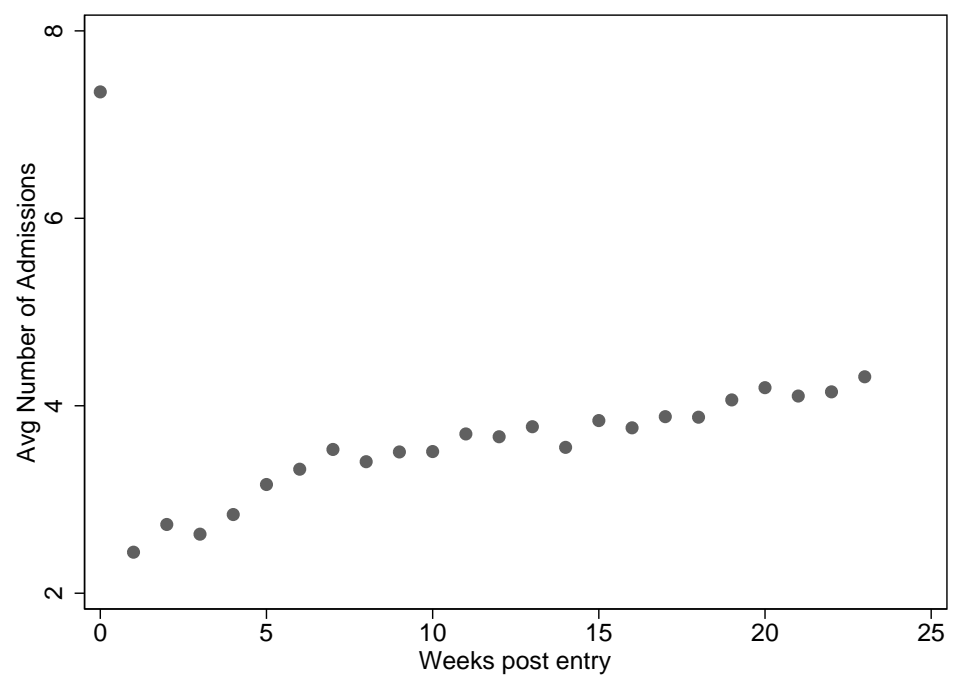

Note: Figure shows the average number of LTCH admissions per week relative to LTCH entry for all LTCH entries from 1998-2014. Admission frequencies were calculated by taking the average number of admissions across all active LTCHs for a given week relative to LTCH entry.

\section{Figure A5: Dates of LTCH Entry Into HSA, As Coded in Different Data Sets}

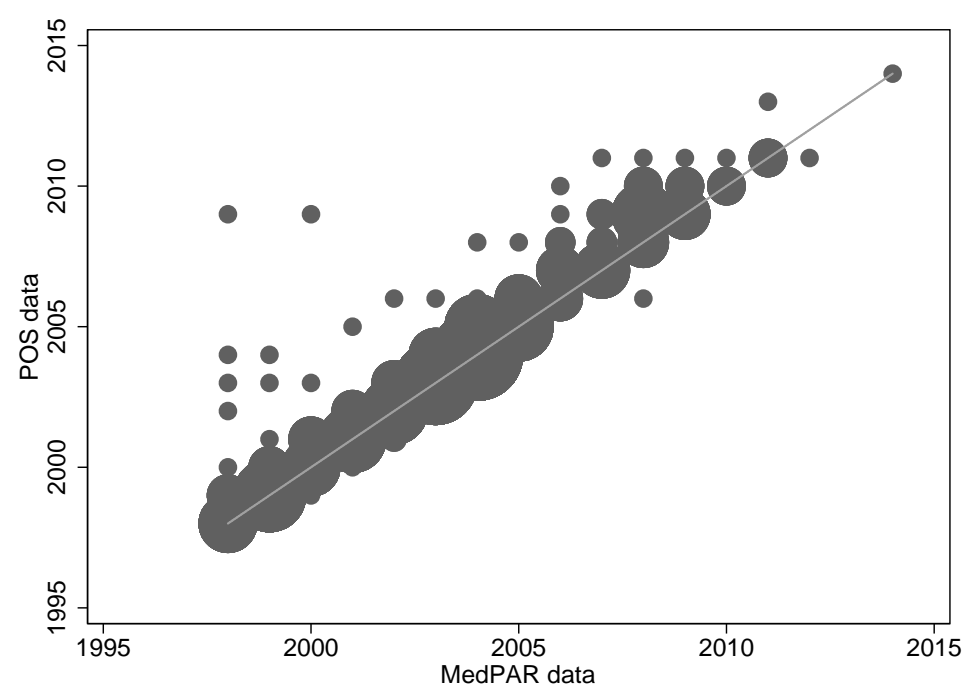

Note: Figure plots entry years using the Provider of Service (POS) file against entry years using MedPAR data from 19982014. The size of each point on the scatter plot is determined by the number of observations for each unique (MedPAR data entry year, POS data entry year) point in order to view the density of matched observations. A 45 degree line is included for reference. 
Figure A6: LTCHs-in-Training: Facility Life

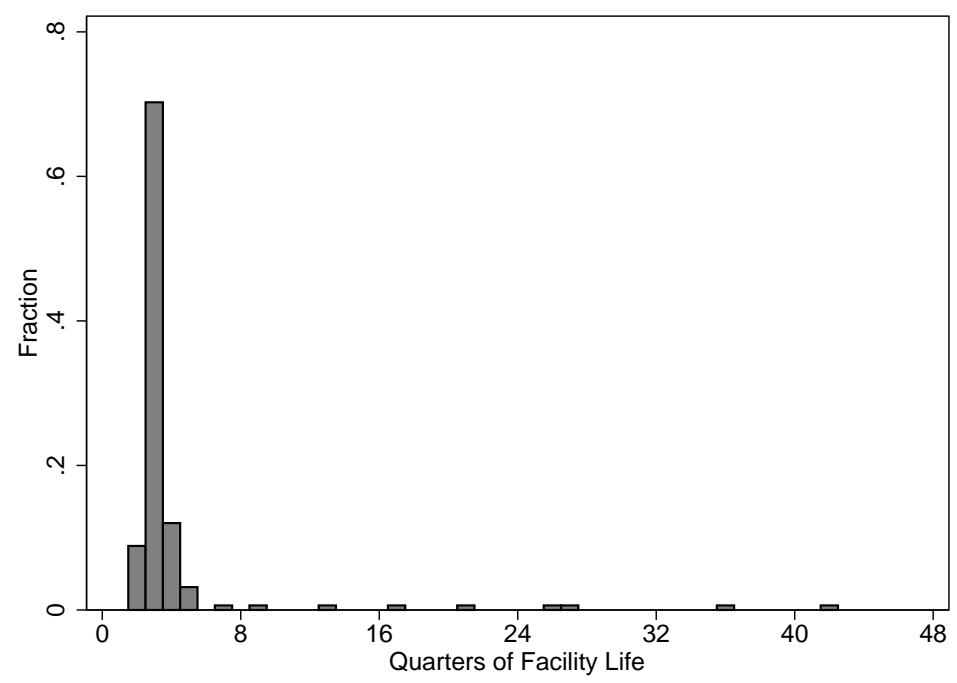

Note: Figure plots a histogram of the number of quarters the LTCHs-in-Training admit patients. Quarters of Facility Life denotes the number of quarters from the first observed admission date to the last observed admission date.

Figure A7: Discharged to ACH

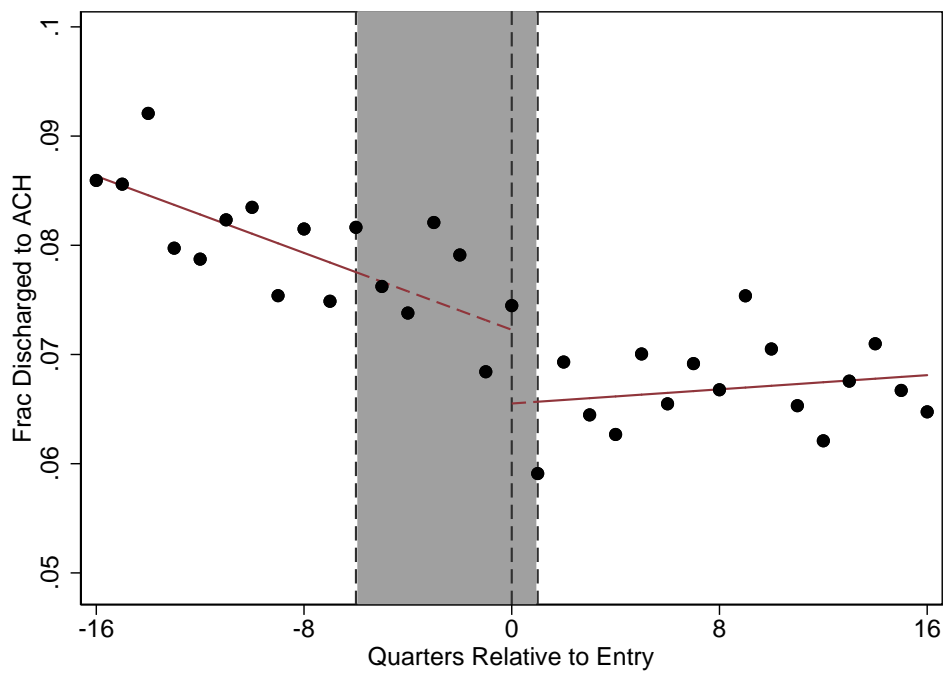

Note: Figure reports estimates of equation (1), estimated on the high $\hat{p}$ sub-sample of the baseline event study sample restricted to the 112 HSAs where we believe we were able to identify an associated LTCH-in-training. Figure displays our estimated function of relative quarter, $r$, and a scatter plot of the average residualized values of Discharge to ACH . Quarters $-6<r<1$ are greyed out because we drop all observations in these quarters. The y-axis reports the share discharged to an $\mathrm{ACH}$ and is scaled so that the mean at $r=-1$ is equal to the mean at $r=-1$ among the 112 HSAs. 
Figure A8: Complexity Parameter vs. R-squared

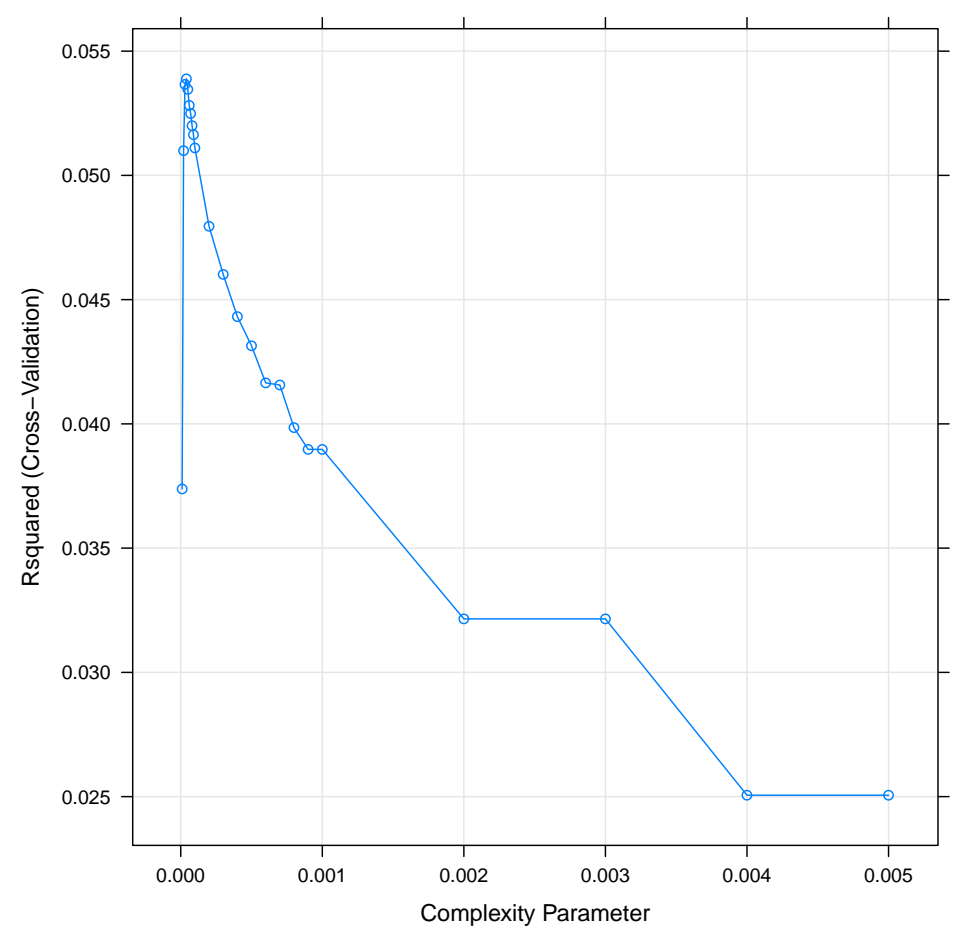

Note: This figure shows a plot of the variation explained $\left(R^{2}\right)$ versus the complexity parameter.

\section{Figure A9: Responsiveness of Procedures to LTCH Entry}

(A) Tracheostomy

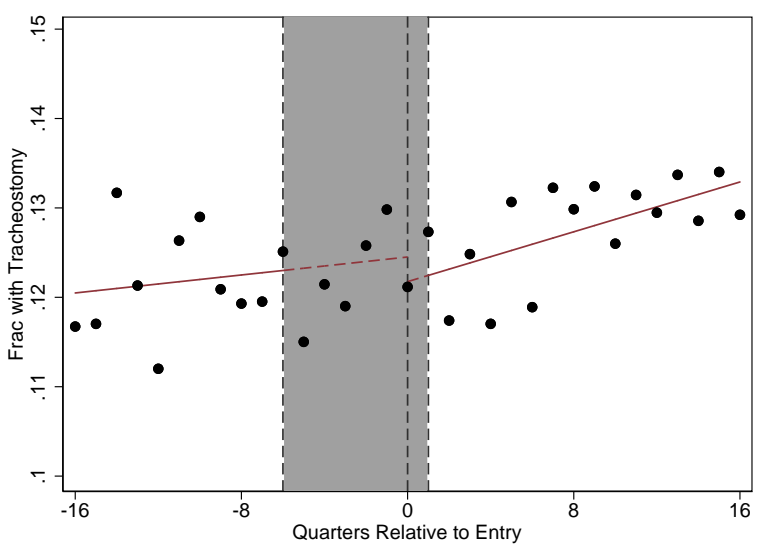

(B) Mechanical Ventilation

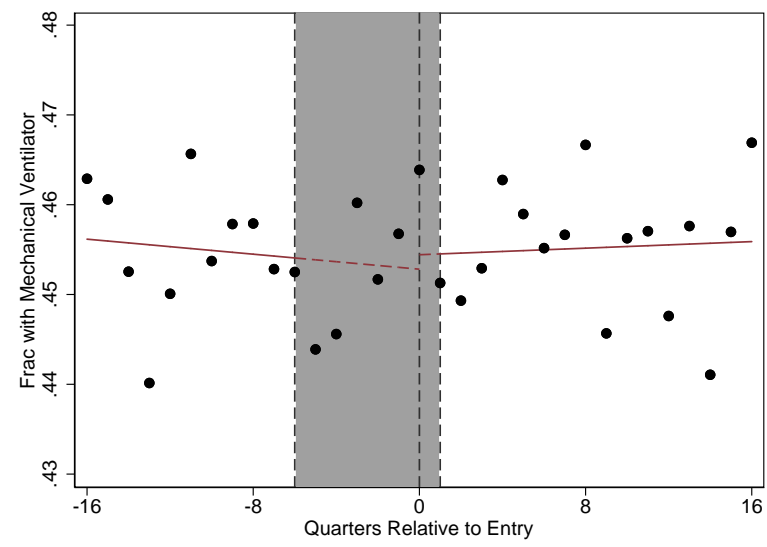

Note: Figure reports estimates of equation (1), estimated on the high $\hat{p}$ sub-sample of the baseline event study sample. Figure displays our estimated function of relative quarter, $r$, and a scatter plot of the average residualized values of tracheostomy, and mechanical ventilation. Quarters $-6<r<1$ are greyed out because we drop all observations in these quarters. The $\mathrm{y}$-axis reports the frequency of the procedure indicated and is scaled so that the mean at $r=-1$ is equal to the outcome mean at $r=-1$ among HSAs. 
Figure A10: Alternative Transition Periods: First Stage

(A) Narrow Transition Period

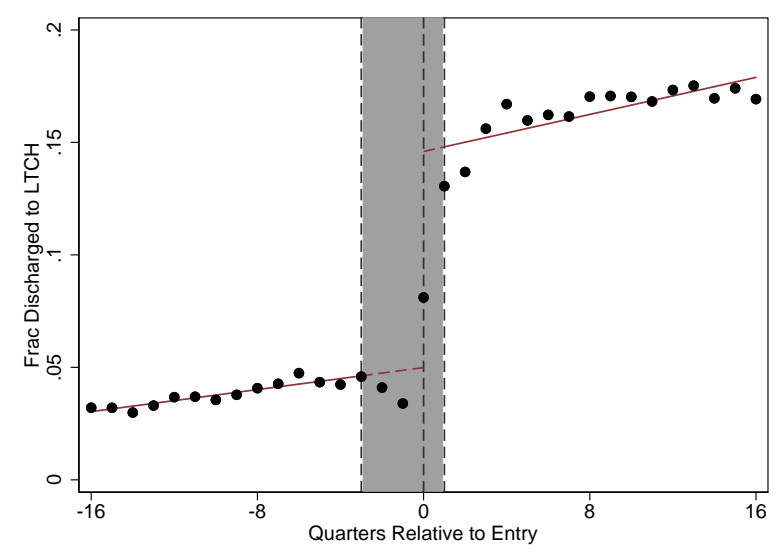

(B) Wide Transition Period

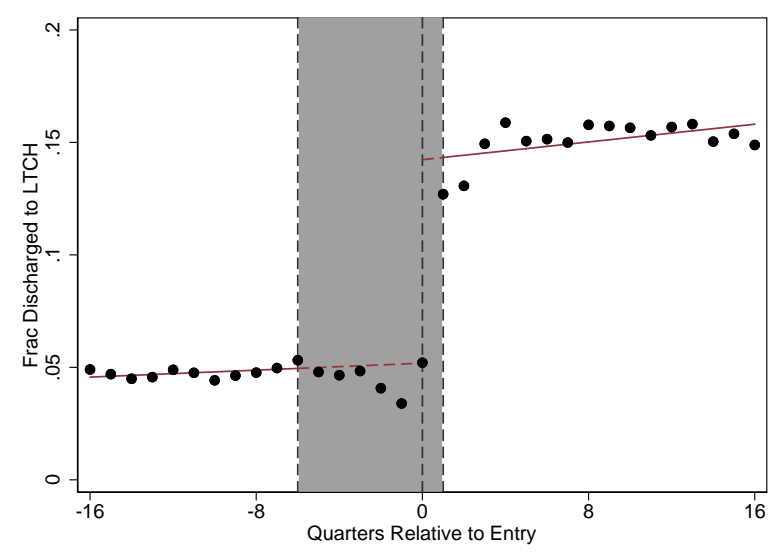

Note: Figure reports estimates of equation (1), estimated on the high $\hat{p}$ sub-sample of the baseline event study sample. Figure displays our estimated function of relative quarter, $r$, and a scatter plot of the average residualized values of the discharge to LTCH indicator. The greyed out quarters are the alternative dropped transition periods, with the narrow transition period defined as $r \in[-2,0]$ and the wide transition period defined as $r=[-5,5]$. The y-axis reports the share discharged to LTCH and is scaled so that the mean at $r=-1$ is equal to the mean at $r=-1$ among HSAs. 


\section{Figure A11: First Stage: Robustness}

(A) Baseline

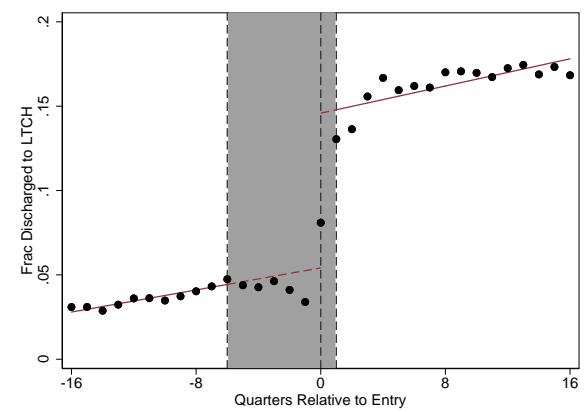

(C) 3 Year Balanced Panel

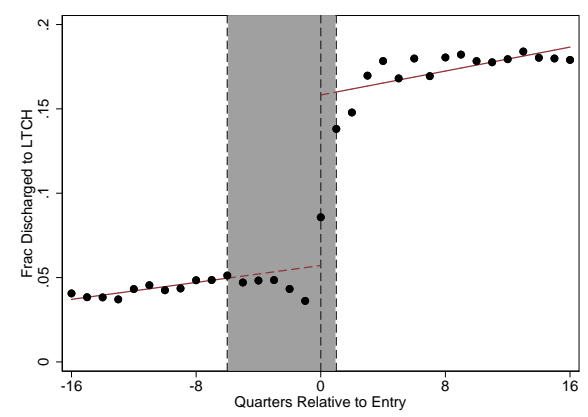

(E) Exclude Dual Eligibles

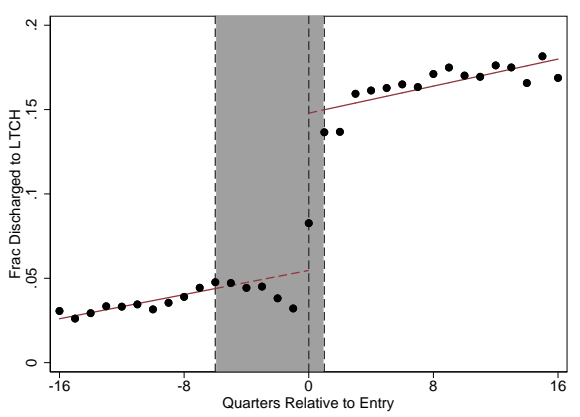

(B) Include HSAs with Prior LTCHs

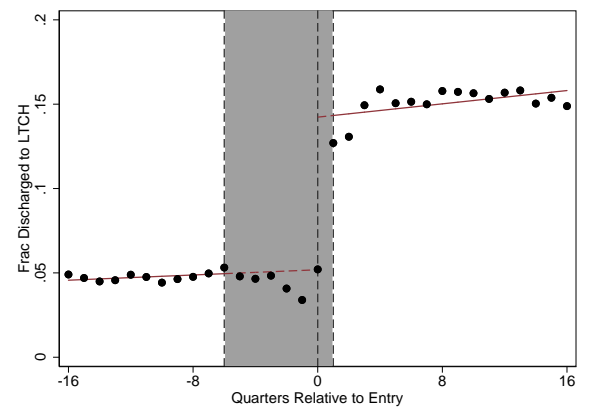

(D) Include Subsequent Entries

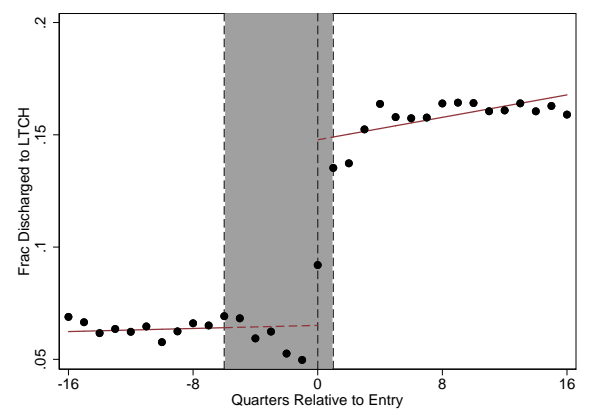

(F) Zip code

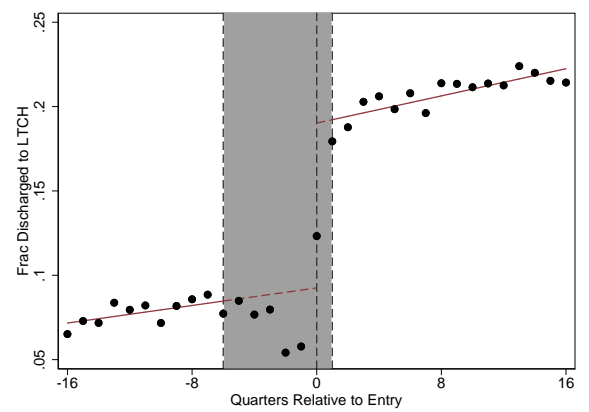

(G) County

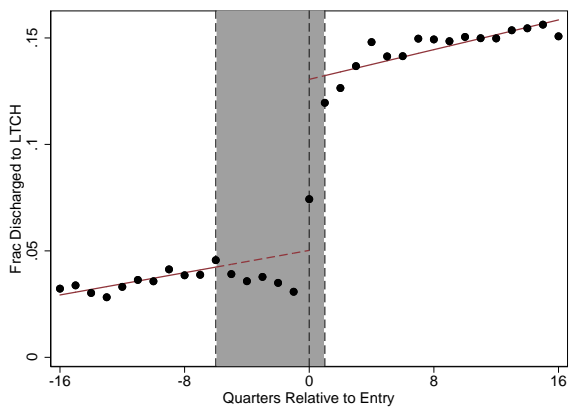

Note: Figure reports estimates of equation (1), estimated on the high $\hat{p}$ sub-sample of various robustness event study samples. Figure displays our estimated function of relative quarter, $r$, and a scatter plot of the average residualized values of the discharge to LTCH indicator. Quarters $-6<r<1$ are greyed out because we drop all observations in these quarters. In panel (A) we present the result from our baseline event study sample. In panel (B) we include all HSAs that already had at least one LTCH as of 1998 as controls. In panel (C) we restrict to all observations with a balanced 3-year panel around entry (i.e. first entries that occurred between 2001 and 2011 and was not followed by an entry or exit within three years). In panel (D) we do not drop subsequent entries or exits, and in panel (E) we restrict to all beneficiary-years in which beneficiaries are not dually eligible for Medicaid. In panels (F) and (G) we use zip code and county as the geographic units. The y-axis repors the share discharged to LTCH and is scaled so that the mean at $r=-1$ is equal to the outcome mean at $r=-1$ among the geographies in each robustness sample. 
Figure A12: Outlier Payments

(A) Baseline Spending

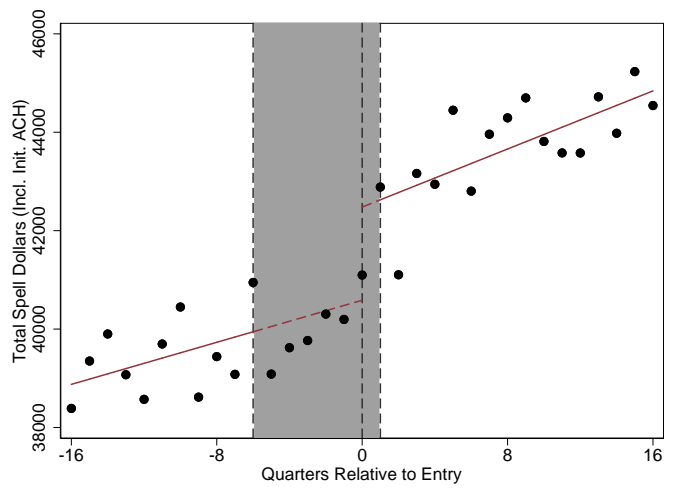

(B) Outlier Spending

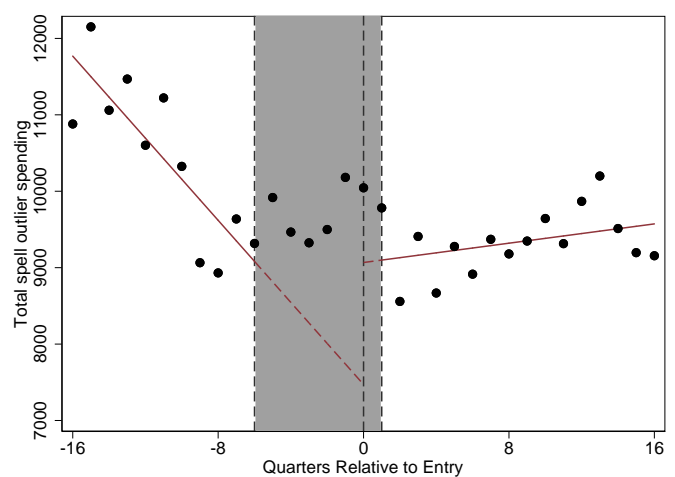

(C) Baseline + Outlier Spending

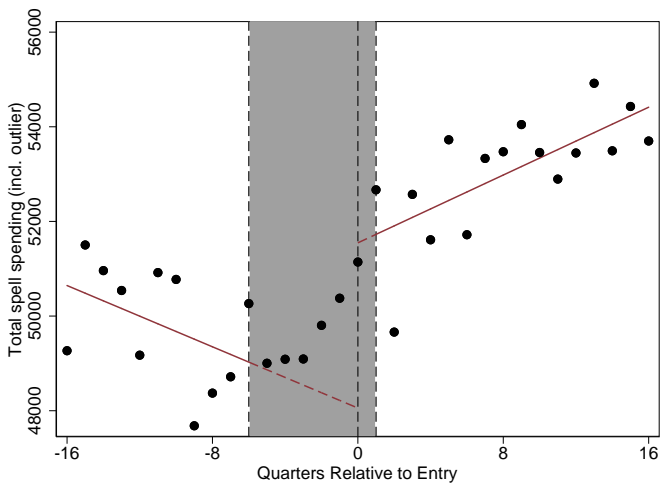

Note: Figure reports estimates of equation (1), estimated on the high $\hat{p}$ sub-sample of the baseline event study sample. The figure displays our estimated function of relative quarter, $r$, and a scatter plot of the average residualized values of the spell utilization by destination. Quarters $-6<r<1$ are greyed out because we drop all observations in these quarters. Panel (A) shows effects on the baseline spending measures, which is the sum of Medicare reimbursements excluding outlier payments, panel (B) shows effects on outlier payments, and panel (C) shows effects on combined baseline and outlier payments. The y-axis reports the spell utilization indicated and is scaled so that the mean at $r=-1$ is equal to the outcome mean at $r=-1$ among HSAs. 
Table A1: Summary Statistics by $\hat{p}$ Bin

\begin{tabular}{|c|c|c|c|c|c|c|c|c|c|c|}
\hline \multirow{2}{*}{ Discharge destination } & \multicolumn{2}{|c|}{$\begin{array}{c}\text { Bin } 1 \\
(1)\end{array}$} & \multicolumn{2}{|c|}{$\begin{array}{c}\operatorname{Bin} 2 \\
(2)\end{array}$} & \multicolumn{2}{|c|}{$\begin{array}{c}\operatorname{Bin} 3 \\
(3)\end{array}$} & \multicolumn{2}{|c|}{$\begin{array}{c}\operatorname{Bin} 4 \\
(4)\end{array}$} & \multicolumn{2}{|c|}{$\begin{array}{c}\operatorname{Bin} 5 \\
(5)\end{array}$} \\
\hline & & & & & & & & & & \\
\hline $\mathrm{LTCH}$ & 0.003 & $(0.056)$ & 0.006 & $(0.080)$ & 0.013 & $(0.115)$ & 0.044 & $(0.205)$ & 0.126 & $(0.332)$ \\
\hline SNF & 0.163 & $(0.370)$ & 0.242 & $(0.428)$ & 0.234 & $(0.424)$ & 0.257 & $(0.437)$ & 0.252 & $(0.434)$ \\
\hline Home/Other & 0.771 & $(0.420)$ & 0.656 & $(0.475)$ & 0.631 & $(0.483)$ & 0.513 & $(0.500)$ & 0.334 & $(0.472)$ \\
\hline LTCH-in-training & 0.000 & $(0.006)$ & 0.000 & $(0.009)$ & 0.000 & $(0.013)$ & 0.001 & $(0.022)$ & 0.001 & $(0.036)$ \\
\hline (other) $\mathrm{ACH}$ & 0.042 & $(0.201)$ & 0.069 & $(0.254)$ & 0.063 & $(0.242)$ & 0.053 & $(0.223)$ & 0.063 & $(0.243)$ \\
\hline Death & 0.021 & $(0.142)$ & 0.027 & $(0.162)$ & 0.059 & $(0.235)$ & 0.134 & $(0.340)$ & 0.224 & $(0.417)$ \\
\hline \multicolumn{11}{|l|}{ Spell days } \\
\hline $\mathrm{LTCH}$ & 0.2 & $(2.5)$ & 0.3 & $(3.2)$ & 0.5 & $(4.3)$ & 1.5 & $(7.6)$ & 4.3 & (13.1) \\
\hline SNF & 6.4 & (18.0) & 9.8 & $(22.1)$ & 9.6 & $(22.5)$ & 10.4 & $(23.3)$ & 12.4 & $(25.9)$ \\
\hline Initiating $\mathrm{ACH}$ & 5.0 & $(4.1)$ & 5.9 & $(4.7)$ & 6.5 & $(5.6)$ & 9.8 & $(8.9)$ & 16.2 & $(14.0)$ \\
\hline Total & 12.6 & $(21.0)$ & 17.6 & $(25.2)$ & 18.3 & $(26.8)$ & 23.7 & (30.3) & 35.8 & $(38.3)$ \\
\hline \multicolumn{11}{|l|}{ Spell spending (\$) } \\
\hline $\mathrm{LTCH}$ & 217 & $(3,160)$ & 342 & $(3,922)$ & 619 & $(5,425)$ & 1,932 & $(10,104)$ & 5,869 & $(18,115)$ \\
\hline SNF & 2,388 & $(6,579)$ & 3,852 & $(8,476)$ & 3,669 & $(8,504)$ & 3,853 & $(8,568)$ & 4,782 & $(10,189)$ \\
\hline Initiating $\mathrm{ACH}$ & 8,770 & $(8,613)$ & 10,221 & $(8,645)$ & 10,027 & $(9,503)$ & 15,113 & $(16,273)$ & 27,583 & $(30,116)$ \\
\hline Total & 12,809 & $(14,272)$ & 16,435 & $(16,107)$ & 16,541 & $(18,109)$ & 23,514 & $(26,609)$ & 42,202 & $(44,769)$ \\
\hline \multicolumn{11}{|l|}{ Patient outcomes } \\
\hline Out-of-pocket spending (\$) & 1,338 & $(2,210)$ & 1,691 & $(2,627)$ & 1,730 & $(2,974)$ & 2,059 & $(3,988)$ & 3,334 & $(6,431)$ \\
\hline Home within 90 days & 0.83 & $(0.38)$ & 0.79 & $(0.41)$ & 0.71 & $(0.45)$ & 0.59 & $(0.49)$ & 0.42 & $(0.49)$ \\
\hline Died within 90 days & 0.14 & $(0.34)$ & 0.13 & $(0.33)$ & 0.22 & $(0.41)$ & 0.32 & $(0.47)$ & 0.44 & $(0.50)$ \\
\hline Mean p-hat & 0.009 & $(0.000)$ & 0.016 & $(0.003)$ & 0.028 & $(0.004)$ & 0.077 & $(0.026)$ & 0.189 & $(0.034)$ \\
\hline Number of Obs. (000s) & \multicolumn{2}{|c|}{3,841} & \multicolumn{2}{|c|}{3,078} & \multicolumn{2}{|c|}{3,236} & \multicolumn{2}{|c|}{2,565} & \multicolumn{2}{|c|}{373} \\
\hline Number of LTCH Discharges & \multicolumn{2}{|c|}{12,204} & \multicolumn{2}{|c|}{19,574} & \multicolumn{2}{|c|}{43,186} & \multicolumn{2}{|c|}{113,211} & \multicolumn{2}{|c|}{47,020} \\
\hline
\end{tabular}

Note: Table shows summary statistics by $\hat{p}$ bin. Each observation is a unique ACH stay. Bins are divided among the baseline event study sample, which drops all observations with $\hat{p} \leq 0.004$, restricts to all HSAs that experience a first entry from 1998-2014, and drops observations at and after the quarter of subsequent entry or LTCH exit. Bins 1 to 3 are quartiles of the $\hat{p}$ distribution, and groups 4 and 5 are based on splitting the top quartile into two groups $(\hat{p}<0.15$ and $\hat{p}>0.15)$. 
Table A2: First Stage and IV by $\hat{p}$ Bin

\begin{tabular}{|c|c|c|c|c|c|c|c|c|c|c|}
\hline Discharge destination & \multicolumn{2}{|c|}{$\begin{array}{c}\text { Bin } 1 \\
(1)\end{array}$} & \multicolumn{2}{|c|}{$\begin{array}{c}\text { Bin } 2 \\
(2)\end{array}$} & \multicolumn{2}{|c|}{$\begin{array}{c}\operatorname{Bin} 3 \\
(3)\end{array}$} & \multicolumn{2}{|c|}{$\begin{array}{c}\text { Bin } 4 \\
(4)\end{array}$} & \multicolumn{2}{|c|}{$\begin{array}{c}\text { Bin } 5 \\
(5)\end{array}$} \\
\hline LTCH & 0.005 & $(0.001)$ & 0.009 & $(0.001)$ & 0.017 & $(0.002)$ & 0.044 & $(0.004)$ & 0.092 & $(0.009)$ \\
\hline SNF & -1.343 & $(0.613)$ & -1.677 & $(0.499)$ & -0.997 & $(0.244)$ & -0.748 & $(0.108)$ & -0.674 & $(0.103)$ \\
\hline Home/Other & 0.262 & $(0.689)$ & 0.432 & $(0.475)$ & -0.204 & $(0.259)$ & -0.288 & $(0.103)$ & -0.244 & $(0.105)$ \\
\hline LTCH-in-training & 0.008 & $(0.005)$ & 0.001 & $(0.003)$ & 0.003 & $(0.002)$ & 0.008 & $(0.004)$ & 0.007 & $(0.006)$ \\
\hline (other) $\mathrm{ACH}$ & 0.217 & $(0.355)$ & 0.456 & $(0.314)$ & 0.180 & $(0.130)$ & 0.030 & $(0.053)$ & -0.035 & $(0.064)$ \\
\hline Death & -0.144 & $(0.171)$ & -0.211 & $(0.119)$ & 0.018 & $(0.104)$ & -0.002 & $(0.072)$ & -0.054 & $(0.101)$ \\
\hline \multicolumn{11}{|l|}{ Spell days } \\
\hline $\mathrm{LTCH}$ & 28.4 & (3.1) & 25.0 & $(2.1)$ & 28.1 & $(1.6)$ & 28.7 & $(1.4)$ & 30.0 & (1.9) \\
\hline SNF & -23.9 & $(27.6)$ & -3.7 & $(21.1)$ & -19.9 & $(11.6)$ & -12.6 & $(5.5)$ & -16.2 & $(6.3)$ \\
\hline Initiating $\mathrm{ACH}$ & -5.8 & $(7.7)$ & -7.7 & $(4.8)$ & -4.8 & $(4.0)$ & -5.7 & $(2.6)$ & -15.5 & $(4.8)$ \\
\hline Total & -5.7 & $(34.6)$ & 17.3 & $(24.0)$ & 4.0 & $(13.9)$ & 9.6 & $(7.0)$ & 1.5 & $(9.4)$ \\
\hline \multicolumn{11}{|l|}{ Spell spending (\$) } \\
\hline $\mathrm{LTCH}$ & 34,141 & $(4,160)$ & 28,373 & $(2,592)$ & 33,445 & $(2,164)$ & 35,367 & $(2,170)$ & 34,210 & $(4,079)$ \\
\hline SNF & 2,855 & $(11,166)$ & 9,179 & $(9,640)$ & $-2,374$ & $(4,368)$ & $-2,621$ & $(2,230)$ & $-5,593$ & $(2,714)$ \\
\hline Initiating $\mathrm{ACH}$ & $-8,730$ & $(19,212)$ & $-6,010$ & $(12,670)$ & $-6,139$ & $(5,476)$ & 4,738 & $(4,504)$ & $-12,256$ & $(9,340)$ \\
\hline Total & 25,426 & $(24,402)$ & 35,264 & $(16,728)$ & 26,120 & $(8,062)$ & 34,874 & $(6,147)$ & 20,649 & $(11,065)$ \\
\hline \multicolumn{11}{|l|}{ Patient outcomes } \\
\hline Out-of-pocket spending (\$) & 499 & $(3,221)$ & 1,786 & $(2,229)$ & 576 & $(1,321)$ & 2,071 & (828) & 3,928 & $(1,381)$ \\
\hline Home within 90 days & 0.19 & $(0.65)$ & 0.24 & $(0.52)$ & -0.21 & $(0.29)$ & -0.27 & $(0.13)$ & -0.04 & $(0.11)$ \\
\hline Died within 90 days & -0.62 & $(0.45)$ & -0.33 & $(0.30)$ & 0.02 & $(0.17)$ & 0.13 & $(0.10)$ & 0.15 & $(0.11)$ \\
\hline Number of Obs. (000s) & \multicolumn{2}{|c|}{3,449} & \multicolumn{2}{|c|}{2,765} & \multicolumn{2}{|c|}{2,931} & \multicolumn{2}{|c|}{2,336} & \multicolumn{2}{|c|}{343} \\
\hline
\end{tabular}

Note: Table displays first stage (top row) and IV estimates (all other rows) for each $\hat{p}$ bin. Standard errors are clustered at the HSA level (186 clusters). Bins 1 to 3 are quartiles of the $\hat{p}$ distribution, and bins 4 and 5 are based on splitting the top quartile into two groups $(\hat{p}<0.15$ and $\hat{p}>0.15)$. 
Table A3: Mortality Horizons

\begin{tabular}{ccccc}
\hline \hline & & \multicolumn{2}{c}{ Baseline Sample } & \\
& Estimate & S.E. & Mean & $\begin{array}{c}\text { Proportional } \\
\text { Change }\end{array}$ \\
\hline Died within & & & \\
30 days & 0.056 & $(0.058)$ & 0.12 & 0.47 \\
60 days & 0.065 & $(0.065)$ & 0.17 & 0.39 \\
90 days & 0.101 & $(0.065)$ & 0.20 & 0.51 \\
180 days & 0.091 & $(0.067)$ & 0.26 & 0.35 \\
365 days & 0.003 & $(0.072)$ & 0.35 & 0.01 \\
Number of Obs. (000s) & \multicolumn{2}{c}{11,824} \\
\hline \hline
\end{tabular}

Note: Table displays IV estimates from equations 4 and 5, estimated on the baseline sample, along with the mean and the implied proportional change in the mortality rate. Mortality is measured from the day of admission to the initiating ACH. Standard errors are clustered at the HSA-bin level. 
Table A4: Heterogeneity by ICU/CCU and Mechanical Ventilator

\begin{tabular}{|c|c|c|c|c|c|c|c|c|c|c|c|c|}
\hline \multirow{2}{*}{ Discharge destination } & \multicolumn{2}{|c|}{$\begin{array}{c}3 \text { or More Days in } \\
\text { ICU/CCU } \\
\text { (1) }\end{array}$} & \multicolumn{2}{|c|}{$\begin{array}{c}\text { Fewer Than } 3 \text { Days in } \\
\text { ICU/CCU } \\
\text { (2) }\end{array}$} & \multicolumn{2}{|c|}{$\begin{array}{c}8 \text { or More Days in } \\
\text { ICU/CCU } \\
\text { (3) }\end{array}$} & \multicolumn{2}{|c|}{$\begin{array}{c}\text { Fewer than } 8 \text { Days in } \\
\text { ICU/CCU } \\
(4)\end{array}$} & \multicolumn{2}{|c|}{$\begin{array}{l}\text { Patient on Ventilator } \\
\text { (5) }\end{array}$} & \multicolumn{2}{|c|}{$\begin{array}{l}\text { Patient Not on } \\
\text { Ventilator } \\
(6)\end{array}$} \\
\hline & & & & & & & & & & & & \\
\hline SNF & -0.689 & $(0.069)$ & -1.015 & $(0.137)$ & -0.700 & $(0.069)$ & -0.901 & $(0.115)$ & -0.673 & $(0.074)$ & -0.851 & $(0.098)$ \\
\hline Home/Other & -0.302 & $(0.067)$ & -0.134 & $(0.141)$ & -0.218 & $(0.066)$ & -0.257 & $(0.113)$ & -0.314 & $(0.082)$ & -0.162 & $(0.094)$ \\
\hline LTCH-in-training & 0.006 & $(0.004)$ & 0.008 & $(0.002)$ & 0.006 & $(0.004)$ & 0.008 & $(0.002)$ & 0.007 & $(0.004)$ & 0.007 & $(0.003)$ \\
\hline (other) $\mathrm{ACH}$ & -0.009 & $(0.043)$ & 0.155 & $(0.064)$ & -0.030 & $(0.043)$ & 0.132 & $(0.057)$ & -0.080 & $(0.049)$ & 0.097 & $(0.048)$ \\
\hline Death & -0.007 & $(0.060)$ & -0.015 & $(0.079)$ & -0.059 & $(0.057)$ & 0.018 & $(0.072)$ & 0.060 & $(0.094)$ & -0.092 & $(0.054)$ \\
\hline \multicolumn{13}{|l|}{ Spell days } \\
\hline LTCH & 28.6 & (1.1) & 28.8 & $(1.3)$ & 30.0 & $(1.2)$ & 27.2 & $(1.1)$ & 31.5 & $(1.5)$ & 27.5 & $(1.0)$ \\
\hline SNF & -12.8 & (3.5) & -19.2 & $(7.2)$ & -15.8 & (3.8) & -14.5 & (5.9) & -19.4 & (4.4) & -11.1 & $(4.9)$ \\
\hline Initiating $\mathrm{ACH}$ & -8.0 & (2.4) & -4.8 & (2.7) & -8.8 & (2.6) & -3.9 & $(2.2)$ & -9.9 & (2.8) & -7.7 & $(2.2)$ \\
\hline Total & 9.1 & (4.9) & 4.0 & (8.8) & 6.4 & $(5.2)$ & 8.8 & $(7.4)$ & 3.0 & (6.9) & 9.4 & $(6.1)$ \\
\hline \multicolumn{13}{|l|}{ Spell spending (\$) } \\
\hline $\mathrm{LTCH}$ & 34,798 & $(1,955)$ & 32,886 & $(1,754)$ & 36,450 & $(2,155)$ & 31,674 & $(1,593)$ & 40,788 & $(2,593)$ & 31,624 & $(1,531)$ \\
\hline SNF & $-4,090$ & $(1,470)$ & $-2,019$ & $(2,718)$ & $-5,285$ & $(1,507)$ & $-1,419$ & $(2,338)$ & $-6,820$ & $(1,763)$ & -988 & $(1,914)$ \\
\hline Initiating $\mathrm{ACH}$ & -235 & $(5,580)$ & $-2,745$ & $(2,923)$ & -570 & $(6,255)$ & 229 & $(2,664)$ & $-4,563$ & $(7,131)$ & -556 & $(3,560)$ \\
\hline Total & 31,935 & $(6,472)$ & 25,818 & $(5,345)$ & 31,108 & $(7,189)$ & 29,780 & $(4,572)$ & 29,033 & $(8,852)$ & 30,695 & $(5,047)$ \\
\hline \multicolumn{13}{|l|}{ Patient outcomes } \\
\hline Out-of-pocket spending (\$) & 3,166 & $(706)$ & 639 & $(960)$ & 3,361 & $(795)$ & 1,151 & $(802)$ & 3,172 & $(977)$ & 2,124 & $(738)$ \\
\hline Home within 90 days & -0.157 & $(0.074)$ & -0.176 & $(0.157)$ & -0.108 & $(0.075)$ & -0.218 & $(0.131)$ & -0.125 & $(0.095)$ & -0.159 & $(0.106)$ \\
\hline Died within 90 days & 0.176 & $(0.070)$ & -0.015 & $(0.120)$ & 0.165 & $(0.070)$ & 0.041 & $(0.101)$ & 0.221 & $(0.097)$ & -0.003 & $(0.077)$ \\
\hline Number of Obs. (000s) & \multicolumn{2}{|c|}{3,297} & \multicolumn{2}{|c|}{8,527} & \multicolumn{2}{|c|}{1,825} & \multicolumn{2}{|c|}{10,000} & \multicolumn{2}{|c|}{924} & \multicolumn{2}{|c|}{10,900} \\
\hline
\end{tabular}

Note: Table displays IV estimates for different splits of the data. Columns 1 and 2 show plots separately by whether the patient spent 3 or more days in an intensive care unit (ICU) or coronary care unit (CCU) prior to LTCH discharge. Columns 3 and 4 show plots separately by whether the patient spent 8 or more days in a ICU / CCU. Columns 5 and 6 split the analysis by whether was placed on a mechanical ventilator at the initiating ACH. Standard errors are clustered at the HSA-bin level. 
Table A5: Heterogeneity by Pre-Entry Discharge and For-Profit Status

\begin{tabular}{|c|c|c|c|c|c|c|c|c|c|c|c|c|}
\hline \multirow{2}{*}{ Discharge destination } & \multicolumn{2}{|c|}{$\begin{array}{c}\text { Below Median } \\
\text { (1) }\end{array}$} & \multicolumn{2}{|c|}{$\begin{array}{c}\text { Above Median } \\
\text { (2) }\end{array}$} & \multicolumn{2}{|c|}{$\begin{array}{l}\text { Bottom Quartile } \\
\text { (3) }\end{array}$} & \multicolumn{2}{|c|}{$\begin{array}{c}\text { Top Quartile } \\
\text { (4) }\end{array}$} & \multicolumn{2}{|c|}{$\begin{array}{l}\text { For Profit } \\
\qquad(5)\end{array}$} & \multicolumn{2}{|c|}{$\begin{array}{l}\text { Not for Profit } \\
\text { (6) }\end{array}$} \\
\hline & & & & & & & & & & & & \\
\hline SNF & -0.713 & $(0.085)$ & -0.765 & $(0.121)$ & -0.655 & $(0.084)$ & -1.391 & (0.209) & -0.745 & $(0.101)$ & -0.824 & $(0.113)$ \\
\hline Home/Other & -0.272 & $(0.088)$ & -0.292 & $(0.115)$ & -0.333 & (0.099) & -0.063 & $(0.189)$ & -0.409 & $(0.100)$ & 0.040 & (0.107) \\
\hline LTCH-in-training & 0.003 & $(0.003)$ & 0.014 & $(0.006)$ & 0.007 & $(0.003)$ & 0.012 & $(0.007)$ & 0.004 & $(0.003)$ & 0.010 & $(0.005)$ \\
\hline (other) ACH & 0.048 & $(0.041)$ & -0.036 & $(0.070)$ & 0.066 & $(0.049)$ & 0.280 & $(0.125)$ & 0.105 & $(0.054)$ & -0.097 & (0.059) \\
\hline Death & -0.067 & $(0.061)$ & 0.080 & $(0.080)$ & -0.085 & $(0.069)$ & 0.161 & $(0.104)$ & 0.045 & $(0.067)$ & -0.129 & $(0.074)$ \\
\hline \multicolumn{13}{|l|}{ Spell days } \\
\hline $\mathrm{LTCH}$ & 29.9 & $(0.9)$ & 27.4 & $(1.8)$ & 27.8 & $(1.0)$ & 30.2 & (3.7) & 29.1 & $(1.3)$ & 28.5 & $(1.6)$ \\
\hline SNF & -16.7 & (5.1) & -9.7 & (5.6) & -14.3 & $(4.6)$ & -32.1 & (9.4) & -10.4 & $(5.0)$ & -19.8 & $(6.4)$ \\
\hline Initiating $\mathrm{ACH}$ & -11.4 & (2.5) & -4.6 & (3.1) & -11.8 & (2.8) & 6.2 & (5.3) & -10.2 & (2.9) & -7.2 & $(2.6)$ \\
\hline Total & 2.3 & (6.7) & 11.6 & (7.1) & 2.0 & (6.1) & 4.4 & $(10.3)$ & 11.6 & $(6.6)$ & -3.1 & $(7.8)$ \\
\hline \multicolumn{13}{|l|}{ Spell spending (\$) } \\
\hline $\mathrm{LTCH}$ & 36,363 & $(1,582)$ & 30,983 & $(3,167)$ & 34,026 & $(1,795)$ & 34,547 & $(5,763)$ & 34,447 & $(2,118)$ & 35,546 & $(3,383)$ \\
\hline SNF & $-3,007$ & $(1,716)$ & $-2,846$ & $(2,917)$ & $-1,965$ & $(1,797)$ & $-13,917$ & $(4,897)$ & 60 & $(2,063)$ & $-6,841$ & $(2,651)$ \\
\hline Initiating $\mathrm{ACH}$ & $-1,925$ & $(4,806)$ & $-1,053$ & $(5,553)$ & -715 & $(4,229)$ & 6,172 & $(8,093)$ & $-6,250$ & $(5,134)$ & 6,501 & $(5,989)$ \\
\hline Total & 31,769 & $(5,791)$ & 24,867 & $(7,797)$ & 31,074 & $(5,744)$ & 22,687 & $(11,466)$ & 33,970 & $(6,287)$ & 25,185 & $(7,997)$ \\
\hline \multicolumn{13}{|l|}{ Patient outcomes } \\
\hline Out-of-pocket spending (\$) & 2,347 & $(824)$ & 2,260 & $(977)$ & 1,478 & $(815)$ & 135 & $(1,371)$ & 3,459 & $(796)$ & 400 & $(1,161)$ \\
\hline Home within 90 days & 0.103 & $(0.090)$ & -0.563 & $(0.164)$ & 0.029 & $(0.107)$ & -0.777 & $(0.317)$ & -0.223 & $(0.111)$ & -0.060 & $(0.148)$ \\
\hline Died within 90 days & 0.014 & $(0.077)$ & 0.276 & $(0.111)$ & 0.012 & $(0.079)$ & 0.226 & $(0.168)$ & 0.192 & $(0.080)$ & -0.062 & (0.109) \\
\hline Number of Obs. (000s) & \multicolumn{2}{|c|}{5,551} & \multicolumn{2}{|c|}{4,590} & \multicolumn{2}{|c|}{2,637} & \multicolumn{2}{|c|}{1,528} & \multicolumn{2}{|c|}{7,599} & \multicolumn{2}{|c|}{4,225} \\
\hline
\end{tabular}

Note: Table displays IV estimates for different splits of the data. Columns 1 and 2 split the sample by whether the MSA had below or above the median LTCH discharge share. Columns 3 and 4 show results for the bottom and top quartiles of LTCH discharge share. Columns 5 and 6 split the analysis by whether the LTCH is a for-profit or non-for-profit organization. In columns 1-4, the pre-entry discharge rate is based on the rate in period $r=-6$. For these columns, we exclude 30 of the 186 MSAs where we do not observe outcomes in period $r=-6$. Standard errors are clustered at the HSA-bin level level. 
Table A6: Selected Features

\begin{tabular}{|c|c|c|}
\hline & CCS Categories & Other \\
\hline Respiratory failure; insufficiency; arrest & Mycoses & Age \\
\hline Septicemia & Intestinal obstruction without hernia & \\
\hline Other aftercare & Complication of device; implant or graft & \\
\hline Nutritional deficiencies & Upper respiratory disease & \\
\hline Complications of surgical procedures of medical care & Acute and unspecified renal failure & \\
\hline Pleurisy; pneumothroax; pulmonary collapse & Other fractures & \\
\hline Secondary malignancies & Urinary tract infections & \\
\hline Acute cerebrovascular disease & Essential hypertension & \\
\hline Pneumonia & Congestive heart failure; nonhypertensive & \\
\hline Intracranial injury & Disorders of lipid metabolism & \\
\hline Chronic ulcer of skin & Bacterial infection & \\
\hline Aspiration pneumonitis; food/vomitus & Peri; endo-; and myocarditis; cardiomyopathy & \\
\hline Infective arthritis and osteomyelitis & Skin and subcutaneous tissue infections & \\
\hline Paralysis & Gangrene & \\
\hline Neck of femur (hip) fracture & Peritonitis and intestinal abscess & \\
\hline Spinal cord injury & Chronic obstructive pulmonary disease and bronchiectasis & \\
\hline
\end{tabular}

Note: Table lists potential predictors for the LTCH discharge regression tree model which were actually selected. 
Table A7: Distribution of $\hat{p}$

\begin{tabular}{lccc}
\hline \hline & $\begin{array}{c}\text { All ACH admissions } \\
(1)\end{array}$ & $\begin{array}{c}\text { High p-hat sample } \\
(2)\end{array}$ & $\begin{array}{c}\text { All close ACH admissions } \\
(3)\end{array}$ \\
\hline Mean & 0.020 & 0.189 & 0.022 \\
S.D. & 0.031 & 0.034 & 0.035 \\
Median & 0.009 & 0.179 & 0.009 \\
Percentile & & & \\
$\quad$ 10th & 0.004 & 0.159 & 0.004 \\
25th & 0.004 & 0.164 & 0.004 \\
75th & 0.024 & 0.206 & 0.025 \\
90th & 0.053 & 0.218 & 0.062 \\
95th & 0.085 & 0.272 & 0.092 \\
99th & 0.173 & 0.291 & 0.181 \\
Discharged to LTCH & 0.009 & 0.110 & 0.022 \\
Number of Obs. (000s) & 163,649 & 2,338 & 32,708 \\
\hline \hline
\end{tabular}

Note: Table presents the distribution of $\hat{p}$ for all $\mathrm{ACH}$ admissions, $\mathrm{ACH}$ admissions with a high predicted probability of LTCH discharge $(\hat{p}>0.15)$, and all ACH admissions with an LTCH within 5 kilometers. 
Table A8: Alternative Transition Periods

\begin{tabular}{|c|c|c|c|c|c|c|}
\hline \multirow{2}{*}{ Discharge destination } & \multicolumn{2}{|c|}{$\begin{array}{l}\text { Baseline } \\
\text { (1) }\end{array}$} & \multicolumn{2}{|c|}{$\begin{array}{c}\text { Narrow transition period } \\
\text { (2) }\end{array}$} & \multicolumn{2}{|c|}{$\begin{array}{l}\text { Wide transition period } \\
\text { (3) }\end{array}$} \\
\hline & & & & & & \\
\hline SNF & -0.791 & $(0.075)$ & -0.676 & $(0.049)$ & -0.716 & $(0.070)$ \\
\hline Home/Other & -0.236 & $(0.073)$ & -0.245 & $(0.052)$ & -0.318 & $(0.073)$ \\
\hline LTCH-in-training & 0.007 & $(0.003)$ & -0.006 & $(0.003)$ & 0.001 & $(0.002)$ \\
\hline (other) $\mathrm{ACH}$ & 0.044 & $(0.040)$ & -0.016 & $(0.027)$ & 0.067 & $(0.038)$ \\
\hline Death & -0.024 & $(0.051)$ & -0.057 & $(0.034)$ & -0.034 & $(0.053)$ \\
\hline \multicolumn{7}{|l|}{ Spell days } \\
\hline $\mathrm{LTCH}$ & 28.9 & $(1.0)$ & 29.2 & $(0.8)$ & 29.0 & (1.1) \\
\hline SNF & -14.3 & (3.9) & -10.5 & (2.4) & -13.2 & (3.6) \\
\hline Initiating $\mathrm{ACH}$ & -8.6 & (2.1) & -7.7 & (1.4) & -9.2 & $(2.2)$ \\
\hline Total & 6.6 & (5.1) & 10.2 & (3.3) & 7.2 & (4.9) \\
\hline \multicolumn{7}{|l|}{ Spell spending (\$) } \\
\hline $\mathrm{LTCH}$ & 34,569 & $(1,708)$ & 35,585 & $(1,307)$ & 35,100 & $(1,691)$ \\
\hline SNF & $-3,024$ & $(1,572)$ & $-2,709$ & $(1,121)$ & $-2,852$ & $(1,578)$ \\
\hline Initiating $\mathrm{ACH}$ & $-2,016$ & $(3,800)$ & -914 & $(2,319)$ & $-2,653$ & $(3,928)$ \\
\hline Total & 29,583 & $(4,810)$ & 31,295 & $(3,240)$ & 29,880 & $(4,657)$ \\
\hline \multicolumn{7}{|l|}{ Patient outcomes } \\
\hline Out-of-pocket spending (\$) & 2,420 & $(640)$ & 3,074 & (469) & 2,486 & $(677)$ \\
\hline Home within 90 days & -0.17 & (0.09) & -0.08 & $(0.06)$ & -0.05 & $(0.09)$ \\
\hline Died within 90 days & 0.10 & $(0.07)$ & 0.02 & $(0.04)$ & 0.08 & $(0.07)$ \\
\hline Number of Obs. (000s) & \multicolumn{2}{|c|}{11,824} & \multicolumn{2}{|c|}{12,438} & \multicolumn{2}{|c|}{10,737} \\
\hline
\end{tabular}

Note: Table displays IV estimates from equations 4 and 5, estimated for the baseline sample, dropping alternative transition period observations. The narrow transition period is defined as $r \in[-2,0]$ and the wide transition period is defined as $r=-5,5]$. Standard errors are clustered at the HSA-bin level (930 clusters). 
Table A9: Robustness

\begin{tabular}{|c|c|c|c|c|c|c|c|c|c|c|c|c|c|c|}
\hline \multirow{2}{*}{ Discharge destination } & \multicolumn{2}{|c|}{$\begin{array}{l}\text { Baseline } \\
\text { (1) }\end{array}$} & \multicolumn{2}{|c|}{$\begin{array}{l}\text { Include HSAs with } \\
\text { prior LTCHs } \\
\text { (2) }\end{array}$} & \multicolumn{2}{|c|}{$\begin{array}{c}3 \text { year balanced panel } \\
\text { (3) }\end{array}$} & \multicolumn{2}{|c|}{$\begin{array}{l}\text { Include subsequent } \\
\text { entries } \\
\text { (4) }\end{array}$} & \multicolumn{2}{|c|}{$\begin{array}{l}\text { Exclude dual eligibles } \\
\qquad \text { (5) }\end{array}$} & \multicolumn{2}{|c|}{$\begin{array}{l}\text { Zip code } \\
\text { (6) }\end{array}$} & \multicolumn{2}{|c|}{$\begin{array}{l}\text { County } \\
\text { (7) }\end{array}$} \\
\hline & & & & & & & & & & & & & & \\
\hline SNF & -0.791 & $(0.075)$ & -0.795 & $(0.075)$ & -0.765 & $(0.081)$ & -0.760 & $(0.074)$ & -0.837 & $(0.082)$ & -0.725 & $(0.086)$ & -0.770 & $(0.090)$ \\
\hline Home/Other & -0.236 & $(0.073)$ & -0.225 & $(0.073)$ & -0.182 & $(0.079)$ & -0.225 & $(0.075)$ & -0.201 & $(0.086)$ & -0.364 & $(0.080)$ & -0.170 & $(0.085)$ \\
\hline LTCH-in-training & 0.007 & $(0.003)$ & 0.008 & $(0.003)$ & 0.006 & $(0.002)$ & -0.009 & $(0.008)$ & 0.007 & $(0.003)$ & 0.002 & $(0.003)$ & 0.007 & $(0.003)$ \\
\hline (other) $\mathrm{ACH}$ & 0.044 & $(0.040)$ & 0.054 & $(0.041)$ & -0.011 & $(0.041)$ & 0.002 & $(0.034)$ & 0.062 & $(0.052)$ & 0.051 & $(0.042)$ & -0.020 & $(0.042)$ \\
\hline Death & -0.024 & $(0.051)$ & -0.042 & $(0.052)$ & -0.048 & $(0.052)$ & -0.009 & $(0.048)$ & -0.031 & $(0.061)$ & 0.037 & $(0.054)$ & -0.047 & $(0.058)$ \\
\hline \multicolumn{15}{|l|}{ Spell days } \\
\hline LTCH & 28.9 & $(1.0)$ & 28.2 & (1.1) & 27.9 & $(1.0)$ & 28.3 & $(1.0)$ & 28.6 & (1.1) & 24.7 & $(1.2)$ & 26.6 & $(1.2)$ \\
\hline SNF & -14.3 & (3.9) & -14.3 & (3.9) & -10.0 & (4.1) & -14.0 & (3.6) & -16.0 & $(4.2)$ & -15.6 & (3.8) & -12.6 & (4.4) \\
\hline Initiating $\mathrm{ACH}$ & -8.6 & (2.1) & -8.7 & (2.1) & -7.2 & (2.0) & -8.0 & (1.8) & -10.1 & (2.3) & -2.9 & (2.1) & -13.1 & (2.5) \\
\hline Total & 6.6 & (5.1) & 5.6 & $(5.1)$ & 10.9 & (5.3) & 5.6 & (4.9) & 4.0 & (5.7) & 7.9 & (5.1) & 2.2 & (5.7) \\
\hline \multicolumn{15}{|l|}{ Spell spending (\$) } \\
\hline LTCH & 34,569 & $(1,708)$ & 33,645 & $(1,903)$ & 34,646 & $(1,866)$ & 33,903 & $(1,892)$ & 33,440 & $(1,811)$ & 27,319 & $(1,913)$ & 34,420 & $(1,828)$ \\
\hline SNF & $-3,024$ & $(1,572)$ & $-3,391$ & $(1,610)$ & $-2,201$ & $(1,423)$ & $-2,965$ & $(1,432)$ & $-2,760$ & $(1,710)$ & $-6,188$ & $(1,705)$ & $-1,182$ & $(1,762)$ \\
\hline Initiating ACH & $-2,016$ & $(3,800)$ & $-1,806$ & $(3,783)$ & 1,829 & $(3,699)$ & $-4,343$ & $(3,331)$ & $-2,355$ & $(4,530)$ & 2,260 & $(3,307)$ & $-7,557$ & $(4,308)$ \\
\hline Total & 29,583 & $(4,810)$ & 28,740 & $(5,001)$ & 34,143 & $(4,973)$ & 24,960 & $(4,582)$ & 29,606 & $(5,925)$ & 23,853 & $(5,253)$ & 26,656 & $(5,493)$ \\
\hline \multicolumn{15}{|l|}{ Patient outcomes } \\
\hline Out-of-pocket spending (\$) & 2,420 & (640) & 2,245 & (656) & 2,559 & (647) & 2,008 & $(610)$ & 2,167 & (718) & 1,514 & (731) & 2,122 & (749) \\
\hline Home within 90 days & -0.172 & $(0.088)$ & -0.169 & $(0.091)$ & 0.014 & $(0.080)$ & -0.217 & $(0.084)$ & -0.058 & $(0.100)$ & -0.299 & $(0.085)$ & 0.089 & $(0.090)$ \\
\hline Died within 90 days & 0.101 & $(0.065)$ & 0.084 & $(0.065)$ & 0.032 & $(0.065)$ & 0.128 & $(0.063)$ & 0.118 & $(0.076)$ & 0.124 & $(0.066)$ & 0.042 & $(0.076)$ \\
\hline Number of Obs. (000s) & \multicolumn{2}{|c|}{11,824} & \multicolumn{2}{|c|}{38,027} & \multicolumn{2}{|c|}{7,993} & \multicolumn{2}{|c|}{15,346} & \multicolumn{2}{|c|}{8,719} & \multicolumn{2}{|c|}{7,919} & \multicolumn{2}{|c|}{12,307} \\
\hline
\end{tabular}

Note: Table displays IV estimates from equations 4 and 5, estimated on the baseline sample. Standard errors are clustered at the geography-bin level. Column 1 presents our baseline results. In column 2 we include all HSAs that already had at least one LTCH as of 1998 as controls. In column 3 we restrict to all observations with a balanced 3-year panel around entry (restricting to entries that occurred between 2001 and 2011). In column 4 we do not drop subsequent entries, and in column 5 we restrict to non-dually eligible beneficiary years. In columns 6 and 7 we use Zip code and county as the geographic units; 230 Zip codes and 148 counties experienced a first LTCH entry. 
Table A10: Pooled versus Interacted First Stage

\begin{tabular}{|c|c|c|c|c|}
\hline Discharge destination & \multicolumn{2}{|c|}{$\begin{array}{l}\text { Baseline } \\
\text { (1) }\end{array}$} & \multicolumn{2}{|c|}{$\begin{array}{l}\text { Non-interacted first stage } \\
\text { (2) }\end{array}$} \\
\hline SNF & -0.791 & $(0.075)$ & -0.926 & $(0.165)$ \\
\hline Home/Other & -0.236 & $(0.073)$ & -0.209 & $(0.170)$ \\
\hline LTCH-in-training & 0.007 & $(0.003)$ & 0.006 & $(0.003)$ \\
\hline (other) $\mathrm{ACH}$ & 0.044 & $(0.040)$ & 0.130 & $(0.095)$ \\
\hline Death & -0.024 & $(0.051)$ & -0.001 & $(0.061)$ \\
\hline \multicolumn{5}{|l|}{ Spell days } \\
\hline $\mathrm{LTCH}$ & 28.9 & $(1.0)$ & 28.1 & $(1.2)$ \\
\hline SNF & -14.3 & (3.9) & -13.5 & (7.3) \\
\hline Initiating $\mathrm{ACH}$ & -8.6 & $(2.1)$ & -4.5 & (2.9) \\
\hline Total & 6.6 & $(5.1)$ & 10.5 & (9.1) \\
\hline \multicolumn{5}{|l|}{ Spell spending (\$) } \\
\hline $\mathrm{LTCH}$ & 34,569 & $(1,708)$ & 33,607 & $(1,966)$ \\
\hline SNF & $-3,024$ & $(1,572)$ & -603 & $(3,462)$ \\
\hline Initiating $\mathrm{ACH}$ & $-2,016$ & $(3,800)$ & 1,196 & $(5,674)$ \\
\hline Total & 29,583 & $(4,810)$ & 34,356 & $(7,821)$ \\
\hline \multicolumn{5}{|l|}{ Patient outcomes } \\
\hline Out-of-pocket spending (\$) & 2,420 & (640) & 1,981 & (925) \\
\hline Home within 90 days & -0.17 & $(0.09)$ & -0.21 & $(0.21)$ \\
\hline Died within 90 days & 0.10 & $(0.07)$ & 0.04 & $(0.10)$ \\
\hline Number of Obs. (000s) & \multicolumn{2}{|c|}{11,824} & \multicolumn{2}{|c|}{11,824} \\
\hline
\end{tabular}

Note: Table displays IV estimates from 4 and 5 (interacted first stage) and equations 2 and 3 (non-interacted first stage) estimated on the baseline sample. Standard errors are clustered at the HSA level for the non-interacted specification (186 clusters) and at the HSA-bin level for the interacted first stage specification (930 clusters). 
Table A11: Summary Statistics: Alternative Spell Definition

\begin{tabular}{|c|c|c|c|c|c|c|}
\hline \multirow{3}{*}{ Spell days } & \multicolumn{4}{|c|}{$1998-2014$} & \multirow{2}{*}{\multicolumn{2}{|c|}{$\begin{array}{c}\text { 2002-2014 } \\
\text { 365-day spells } \\
\text { (3) }\end{array}$}} \\
\hline & \multicolumn{2}{|c|}{$\begin{array}{c}\text { Continuous spells } \\
\text { (1) }\end{array}$} & \multicolumn{2}{|c|}{$\begin{array}{l}\text { 365-day spells } \\
\text { (2) }\end{array}$} & & \\
\hline & & & & & & \\
\hline LTCH & 0.7 & $(5.1)$ & 1.2 & $(7.2)$ & 1.4 & $(7.8)$ \\
\hline SNF & 9.0 & $(21.5)$ & 14.5 & $(29.2)$ & 15.3 & $(30.2)$ \\
\hline Initiating $\mathrm{ACH}$ & 6.9 & $(6.7)$ & 6.9 & $(6.7)$ & 6.7 & $(6.4)$ \\
\hline Total & 18.0 & $(26.5)$ & 32.2 & (39.1) & 33.0 & $(40.3)$ \\
\hline Home health care & & & & & 41.5 & $(79.9)$ \\
\hline Hospice & & & & & 7.1 & (34.1) \\
\hline Total (incl. home health and hospice) & & & & & 81.6 & (99.9) \\
\hline Spell spending & & & & & & \\
\hline LTCH & 843 & $(6,657)$ & 1,500 & $(9,161)$ & 1,821 & $(10,078)$ \\
\hline SNF & 3,404 & $(8,078)$ & 5,404 & $(10,813)$ & 5,805 & $(11,337)$ \\
\hline Initiating $\mathrm{ACH}$ & 11,201 & $(12,342)$ & 11,201 & $(12,342)$ & 11,382 & $(12,397)$ \\
\hline Total & 17,519 & $(20,713)$ & 32,448 & $(33,488)$ & 33,955 & $(35,026)$ \\
\hline Home health care & & & & & 2,698 & $(5,058)$ \\
\hline Hospice & & & & & 1,303 & $(5,676)$ \\
\hline Total (incl. home health and hospice) & & & & & 37,956 & $(36,627)$ \\
\hline Number of Obs. (000s) & & & & & & \\
\hline
\end{tabular}

Note: Each observation is a unique ACH stay. Includes all HSAs that experience a first entry from 1998-2014, dropping observations at and after the quarter of subsequent entry or LTCH exit and excluding all observations with the minimum $\hat{p}$ value (the Baseline Sample). Column 1 summarizes outcomes using the continuous spell definitions for utilization used in our baseline utilization analysis; column 1 is the same as the baseline column in Table 3 of the main exhibits. All subsequent columns represent a single deviation from the baseline, as indicated. Column 2 summarizes utilization using a 365-days post admission spell, summing all days and spending at an ACH, LTCHs, SNFs, IRFs, or LTCHs-in-Training with admission dates within 365 days of the admission date at the index acute care stay. Column 3 restricts to 2002-2014 (the years when we have hospice and home health claims data). For these years, we incorporate utilization at home health and hospice into the 365-day post admission spell definition. 
Table A12: Event Study Estimates: Alternative Spell Definition

\begin{tabular}{|c|c|c|c|c|c|c|}
\hline \multirow[b]{3}{*}{ Discharged to Home/Other } & \multicolumn{4}{|c|}{$1998-2014$} & \multirow{2}{*}{\multicolumn{2}{|c|}{$\begin{array}{c}\text { 2002-2014 } \\
\text { 365-day spells } \\
\text { (3) }\end{array}$}} \\
\hline & \multicolumn{2}{|c|}{$\begin{array}{c}\text { Continuous spells } \\
\text { (1) }\end{array}$} & \multicolumn{2}{|c|}{$\begin{array}{l}\text { 365-day spells } \\
\text { (2) }\end{array}$} & & \\
\hline & & & & & -0.327 & $(0.100)$ \\
\hline Home health care & & & & & 0.015 & $(0.063)$ \\
\hline Hospice & & & & & -0.070 & (0.039) \\
\hline Home (no care) & & & & & -0.114 & $(0.071)$ \\
\hline Other & & & & & -0.158 & $(0.059)$ \\
\hline \multicolumn{7}{|l|}{ Spell days } \\
\hline LTCH & 28.9 & (1.0) & 47.7 & $(1.2)$ & 49.5 & (1.6) \\
\hline SNF & -14.3 & (3.9) & -26.8 & (4.9) & -21.7 & $(6.0)$ \\
\hline Initiating ACH & -8.6 & (2.1) & -8.6 & (2.1) & -5.8 & (2.6) \\
\hline Total & 6.6 & (5.1) & 2.7 & $(6.6)$ & 7.8 & (7.9) \\
\hline Home health care & & & & & 19.6 & $(18.0)$ \\
\hline Hospice & & & & & -1.2 & $(5.5)$ \\
\hline Total (incl. home health and hospice) & & & & & 26.2 & $(21.2)$ \\
\hline \multicolumn{7}{|l|}{ Spell spending } \\
\hline LTCH & 34,569 & $(1,708)$ & 56,499 & $(1,868)$ & 60,876 & $(2,106)$ \\
\hline SNF & $-3,024$ & $(1,572)$ & $-7,128$ & $(2,102)$ & $-8,085$ & $(2,310)$ \\
\hline Initiating $\mathrm{ACH}$ & $-2,016$ & $(3,800)$ & $-2,016$ & $(3,800)$ & 9,841 & $(4,470)$ \\
\hline Total & 29,583 & $(4,810)$ & 40,507 & $(7,022)$ & 55,873 & $(8,065)$ \\
\hline Home health care & & & & & 916 & $(1,191)$ \\
\hline Hospice & & & & & -313 & (917) \\
\hline Total (incl.home health and hospice) & & & & & 56,477 & $(8,305)$ \\
\hline Number of Obs. (000s) & \multicolumn{2}{|c|}{11,824} & \multicolumn{2}{|c|}{11,431} & \multicolumn{2}{|c|}{5,997} \\
\hline
\end{tabular}

Note: Table displays IV estimates from equations 4 and 5, estimated on the baseline sample. Standard errors are clustered at the HSA-bin level. 
Table A13: Event Study Estimates: Outlier Payments

\begin{tabular}{|c|c|c|c|c|c|c|}
\hline \multirow{2}{*}{ Spell spending (\$) } & \multicolumn{2}{|c|}{$\begin{array}{l}\text { Baseline } \\
\text { (1) }\end{array}$} & \multicolumn{2}{|c|}{$\begin{array}{l}\text { Outlier } \\
\text { (2) }\end{array}$} & \multicolumn{2}{|c|}{$\begin{array}{l}\text { Baseline + Outlier } \\
\text { (3) }\end{array}$} \\
\hline & & & & & & \\
\hline $\mathrm{LTCH}$ & 34,569 & $(1,708)$ & 5,155 & (791) & 39,724 & $(1,922)$ \\
\hline SNF & $-3,024$ & $(1,572)$ & 178 & (57) & $-2,846$ & $(1,575)$ \\
\hline Initiating $\mathrm{ACH}$ & $-2,016$ & $(3,800)$ & 4,429 & $(8,184)$ & 2,413 & $(9,836)$ \\
\hline Total & 29,583 & $(4,810)$ & 10,884 & $(8,489)$ & 40,466 & $(10,245)$ \\
\hline
\end{tabular}

Note: Table displays IV estimates from equations 4 and 5, estimated on the baseline sample. Column 1 shows effects on the baseline spending measures, which is the sum of Medicare reimbursements excluding outlier payments. Column 2 shows effects on outlier payments. Column 3 shows effects on combined baseline and outlier payments. Standard errors are clustered at the HSA-bin level. 